UNIVERSITÉ DU QUÉBEC

\author{
MÉMOIRE \\ PRÉSENTÉ À \\ L'UNIVERSITÉ DU QUÉBEC À CHICOUTIMI \\ COMME EXIGENCE PARTIELLE
}

DE LA MAÎTRISE EN LETTRES

\title{
PAR \\ NICOLAS CÔTÉ
}

FICTION, RÉCIT ET LECTURE VIDÉOLUDIQUES :

UNE APPROCHE LITTÉRAIRE AU JEU ÉLECTRONIQUE

JANVIER 2012 



\section{RÉSUMÉ}

Le jeu électronique constitue désormais une industrie culturelle majeure, qui n'a de cesse de croître. Ce mémoire propose de l'étudier sous un angle qui, jusqu'à tout récemment, lui était contesté : la fiction, le récit et la lecture, un angle issu du paradigme littéraire. Car le jeu électronique, s'il s'agit d'abord d'un jeu, peut aussi présenter et développer un monde fictionnel complexe, comme ce mémoire cherche à le défendre et à le détailler.

Le travail ici présenté se concentre sur l'établissement d'un cadre théorique de référence, et sur l'application de ce dernier à un éventail restreint de cas, servant à illustrer une diversité d'approches aux jeux électroniques embrassant leur dimension fictionnelle et narrative. Divisé en trois chapitres, ce mémoire traite séparément de trois aspects essentiels de l'expérience de la fiction, et de la façon dont ils se déploient dans les trois jeux analysés: Fallout 3, World of Warcraft: Wrath of the Lich King ainsi que Valkyria Chronicles.

Le premier chapitre est consacré à la dimension des règles de la fiction. Il cherche à défendre une pensée holistique $d u$ jeu électronique, qui comprend une dimension ludique et une dimension fictionnelle. Le cadre d'analyse qui y est développé se base essentiellement sur la littérature concernant le jeu, ainsi que sur une approche systémique du texte inspirée de Lector in Fabula d'Umberto Eco.

Le deuxième chapitre est consacré à la structure du récit. Basé sur la pensée du récit développée par Bertrand Gervais dans son ouvrage Récits et actions, il tente de montrer que l'expérience de jeu peut être interprétée de la même façon que l'expérience du récit, et que les séquences et enchaînements propres aux deux peuvent être définis à l'intérieur d'un même paradigme.

Le dernier chapitre est consacré à la lecture comme rapport personnel à l'univers fictionnel et ludique. Centré autour d'une conception de la lecture issue de la recherche d'Espen Aarseth résumée dans Cybertext; Perspectives on Ergodic Literature, il veut montrer que la place particulière occupée par le joueur, à la fois spectateur et agent, permet au jeu électronique d'offrir de nombreuses possibilités d'interaction, de réception et d'interprétation. Afin de soutenir l'idée du joueur-agent, le chapitre emprunte à la vision systémique de Marie-Laure Ryan, exposée dans Possible Worlds, Artificial Intelligence, qui cherche à appliquer une compréhension de la fiction à un agent interne à celle-ci.

MOTS-CLÉS : Jeu, jeu vidéo, jeu électronique, vidéoludique, littérature, fiction, narration, récit, lecture, systémique. 


\section{REMERCIEMENTS}

Pour mon directeur, qui n'a jamais accepté de moi que le meilleur de ce que je pouvais lui offrir. L'épreuve du feu, c'était un euphémisme! Nicolas, merci!

Pour celle avec qui j'ai tout partagé, les hauts et les bas, les joies et les peines, qui m'a soutenu, encouragé, mis au travail forcé - et qui est toujours restée. Anne-Julie, merci!

Pour mes parents, qui grâce à leur soutien moral et financier m'ont permis d'aller jusqu'au bout de ce projet de fou. Régis, Rose-Ange, merci!

Pour ceux qui ont vécu avec moi tout le processus de près, qui m'ont rappelé qu'on vit tous la même solitude dans la rédaction. Philippe, Sophie, merci!

Pour ma belle-famille, qui m'a hébergé quand j'en ai eu besoin, et qui m'a encouragé du début à la fin de cette aventure. Claude, Lili, Rafaël, Pierre-Marc, merci!

Pour les nombreuses et brillantes personnes que j'ai rencontrées pendant la maîtrise, avec qui j’ai exulté et râlé. Société des Sexy Historiens Extraordinaires, Shack à Beaulieu, merci!

Pour mes collègues de travail, qui achèvent enfin de m'entendre dire " $j$ 'achève ", et pour ma coordonatrice, un puits infini de compréhension. Château Ramezay, Sara, merci!

Pour tous les vieux amis, ceux qui étaient là avant, et qui le sont toujours. David, Alexandre, Catherine, Maxime, Virginie, Jean-Philippe, Jean-François, Pascal, merci!

Pour les nombreux professeurs qui m'ont inspiré, qui ont su voir ce que je valais, surtout quand je ne le voyais pas. Professeurs de lettres du Collège d'Alma et de l'UQAC, merci!

Pour tous ceux que je n'ai pas nommés, famille et amis, proches ou éloignés, qui ont contribué par leur soutien de près ou de loin à ce projet. Vous savez qui vous êtes, merci! 


\section{TABLE DES MATIÈRES}

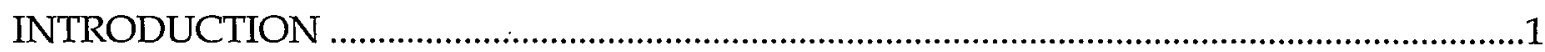

\section{CHAPITRE I}

FICTION ET JEU - ENSEMBLE(S) DE RĖGLES VIDÉOLUDIQUES .................................11

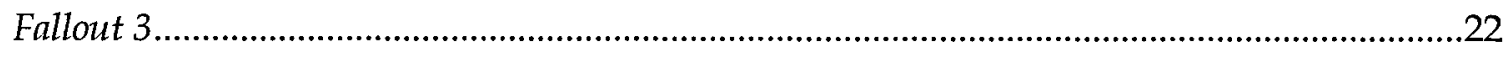

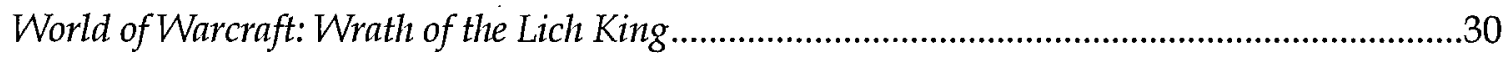

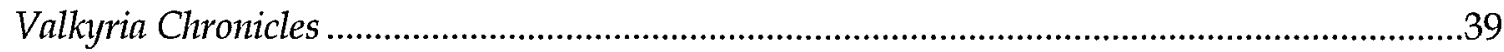

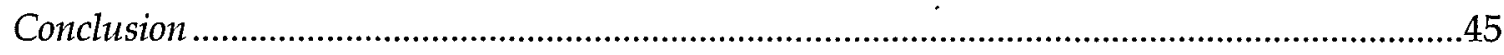

\section{CHAPITRE II}

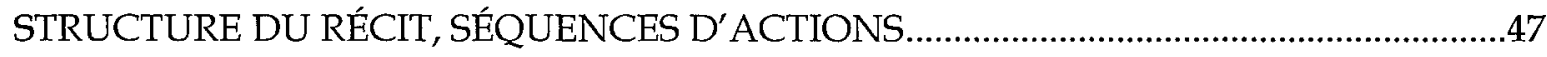

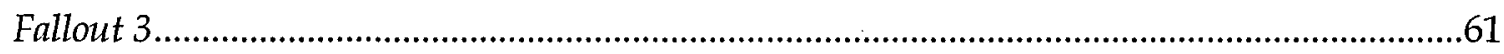

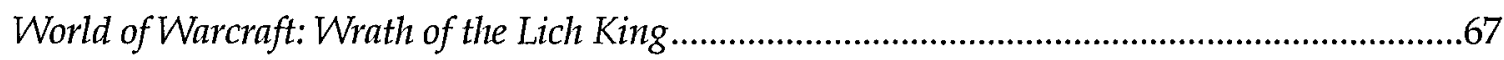

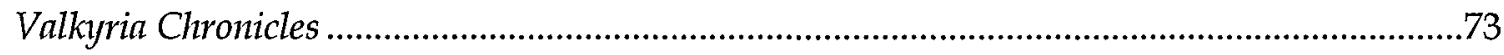

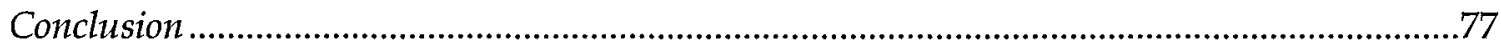

\section{CHAPITRE III}

UN MONDE DE POSSIBILITÉS - LECTURE VIDÉOLUDIQUE .........................................79

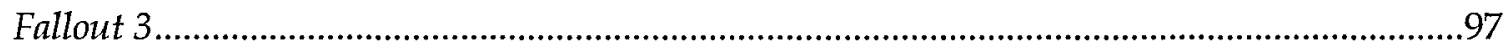

World of Warcraft: Wrath of the Lich King ...........................................................................104

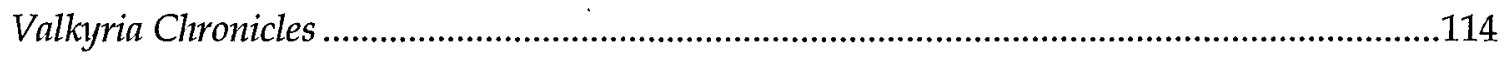

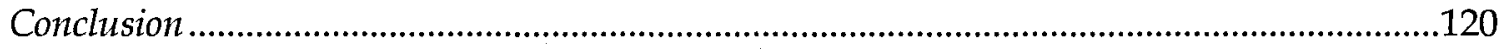

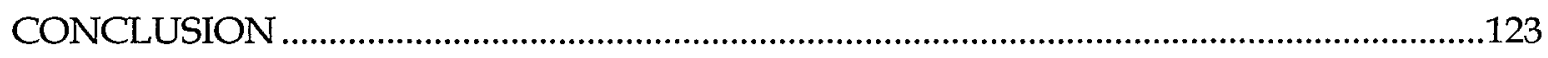

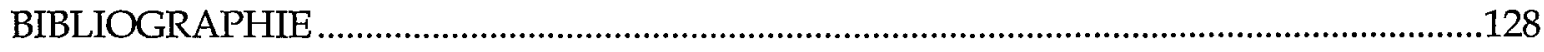




\section{INTRODUCTION}

Dans le cadre de ce mémoire, nous tenterons d'aborder deux sujets d'étude hautement différents : le jeu électronique et la littérature. À première vue difficiles à réconcilier, les deux ont pourtant nombre de traits en commun, et se sont imposés à nous grâce au même élément - leur narrativité. Il nous est donc apparu naturel d'employer (autant que possible) une approche analytique littéraire pour parler du jeu, abordé comme une autre forme du récit. Les limites d'une approche purement littéraire se sont toutefois rapidement fait sentir, étant donné les grandes différences entre les jeux électroniques et les textes écrits. Ainsi est apparu clairement l'objectif de notre mémoire : développer un vocabulaire et une approche adaptés à ce nouvel objet d'études - une approche du récit vidéoludique qui, inspirée de l'analyse littéraire, pourrait rendre compte des particularités de ce mode de représentation.

La première question qui s'impose est la suivante : «qu'est-ce qu'un jeu électronique? ». Commençons par rappeler l'évidence : il s'agit d'abord d'un jeu, et son fonctionnement est électronique. Le jeu, en tant qu'objet social et culturel, a déjà été étudié et décortiqué : on pense aux travaux de Huizinga ${ }^{1}$ ou de Caillois ${ }^{2}$ qui demeurent des incontournables. On y parle du jeu ainsi que l'acte de jouer comme d'une activité qui « dépasse [...] les limites d'une activité exclusivement biologique $»^{3}$ et qui devient ainsi une «fonction riche de

\footnotetext{
1 Johan Huizinga - Homo Ludens: Essai sur la fonction sociale du jeu, Éditions Gallimard, Paris, 1951.

2 Roger Caillois - Les Jeux et les hommes : le masque et le vertige, Éditions Gallimard (Folio Essais), Paris, 1992. (Édition originale 1958)

3 Johan Huizinga - Homo Ludens: Essai sur la fonction sociale du jeu, p.16.
} 
sens. »4 Plus encore, on peut y dégager une vision $\mathrm{du}$ «jeu comme forme d'activité, comme forme pourvue de sens, et comme fonction sociale. ${ }^{5}$ L'activité peut ensuite se décliner de bien des façons, et est définie par Caillois comme essentiellement libre, séparée, incertaine, improductive, réglée et fictive. ${ }^{6}$ De cette définition nous retenons pour l'heure la notion de règle, car « $l^{\prime}$ essence du jeu est de respecter les règles. $»^{7} C^{\prime}$ est là que se situe le rôle tenu par la nature électronique des jeux qui nous préoccupent : le fonctionnement du jeu (le respect de ses règles) passe ainsi par le support électronique - par un programme informatique. Il nous apparaît dès lors intéressant de noter que, si la notion de règles est cruciale pour la pensée du jeu, elle n'est pas non plus étrangère à la fiction, qui peut être comprise, dans une approche systémique, comme définie également par un ensemble de règles. Nous pouvons donc déjà voir un rapprochement entre la fiction et le jeu, que nous approfondirons dans ce mémoire.

Outre l'appellation de «jeu électronique », nous utiliserons la notion de « vidéoludique », qui, bien qu'elle recoupe le même ensemble de jeux, rappelle étymologiquement une autre particularité de cet ensemble : la représentation graphique (ou «vidéo »). Nous croyons cependant plus pertinent de mettre l'accent non pas sur cette particularité de représentation, mais sur le mode de fonctionnement sous-jacent, informatique. C'est pourquoi nous avons opté pour la notion de jeux électroniques plutôt que de jeux vidéo. Le qualificatif «vidéoludique» pourra nous servir de synonyme élégant, sans pour autant

\footnotetext{
4 Ibid.

5 Ibid., p.20.

${ }^{6}$ Roger Caillois - Les Jeux et les hommes : le masque et le vertige, p.42-43.

${ }^{7}$ Johan Huizinga - Homo Ludens: Essai sur la fonction sociale du jeu, p.93.
} 
évacuer la caractéristique essentielle de notre objet d'études, qui demeure sa nature de jeu, et son fonctionnement électronique.

Il faut admettre que nulle part dans cette définition l'idée de récit ou de représentation a été soulevée, et pour cause. Le jeu n'est pas essentiellement un mode de représentation car si «tout jeu signifie quelque chose ${ }^{8}$, il n'est pas nécessaire que cette signification prenne la forme d'un récit, pas plus dans le jeu électronique que dans d'autres types de jeux. Comme notre mémoire porte sur le récit vidéoludique, cependant, il nous apparaît essentiel de cerner quel sous-ensemble utilise la mise en récit, et pour ce faire, nous désirons établir une classification des jeux électroniques en « genres. »

La question du genre, quand on parle de jeux électroniques, est épineuse. Limités par une technologie informatique qui en était encore à ses balbutiements, les premiers jeux électroniques ne se prêtaient guère à une codification semblable. Il faudra attendre les consoles électroniques et les ordinateurs personnels, au début des années 1980, pour que s'organise l'industrie du jeu électronique, et que se diversifie l'offre. C'est alors que se développeront différents « genres » qui demeurent présents dans le jargon et les méthodes de l'industrie actuelle. Si certains genres demeurent nouveaux ou rares (les jeux publicitaires, par exemple), d'autres font écho aux premiers jeux sur console : les jeux d'aventure, de rythme, de plateforme, de rôle, de sport, de stratégie, etc. 9 Nous n'avons

\footnotetext{
8 Ibid., p.16.

${ }^{9}$ Steve L. Kent - The Ultimate History of Video Games: From Pong to Pokemon - The Story Behind the Craze That Touched Our Lives and Changed the World, Three Rivers Press, New York, 2001.
} 
pas pour ambition de faire une typologie complète; il s'agit plutôt pour nous d'identifier le(s) genre(s) le(s) plus apte(s) à être abordé(s) du point de vue du récit. Nous avons choisi de nous consacrer principalement aux jeux de rôle (JDR, ou en anglais, RPG), inspirés des jeux sur table, qui supposent la prise en charge par le joueur d'un (ou de plusieurs) personnage(s), dont il doit tenir le(s) rôle(s). Surtout, il s'agit d'un genre fortement associé à l'avancement d'un personnage à travers les choix effectués par le joueur, à la représentation d'un univers fictionnel et à sa mise en intrigue.

Le développement des jeux électroniques étant constamment soumis aux aléas des avancées technologiques, et leurs genres se trouvant souvent redéfinis, nous avons opté, pour les besoins de notre analyse, pour trois jeux étant parus sensiblement au même moment (à l'automne 2008) et présentés comme des JDR. Chacun exemplifie cependant un différent sous-genre de cette grande famille. Les trois œuvres retenues demeurent ainsi liées, mais présentent une approche différente du jeu comme du récit. Nous avons opté, donc, pour les jeux suivants: Fallout $3^{10}$, World of Warcraft: Wrath of the Lich King11 et Valkyria Chronicles ${ }^{12}$.

Fallout 3 est généralement présenté comme un hybride entre jeu de tir et jeu de rôle - c'està-dire qu'il emprunte de nombreux éléments à chacun. Il s'apparente à un jeu de tir par son interface à la première personne et son rythme de jeu en temps réel, mais demeure essentiellement un jeu de rôle de par l'accent qu'il met sur le développement du

10 Bethesda Game Studios - Fallout 3, Bethesda Softworks, 2008.

11 Blizzard Entertainment - World of Warcraft: Wrath of the Lich King, Blizzard Entertainment, 2008.

12 Sega - Valkyria Chronicles, Sega, 2008. 
personnage. L'œuvre est par ailleurs intéressante par son appartenance à une famille de jeux de rôles surnommés « bacs à sable » ${ }^{13}$ pour leur monde fictionnel immense, qui laisse une grande liberté d'exploration, d'action et d'expérimentation au joueur.

World of Warcraft: Wrath of the Lich King est une extension pour le jeu massivement multijoueurs en ligne World of Warcraft, originellement paru en 2004. Plus qu'une simple addition de contenu, il s'agit d'une redéfinition du jeu, ainsi que d'une remise en question de ses systèmes de fonctionnement; aussi pouvons-nous le considérer comme une œuvre auto-suffisante. Les jeux de rôles massivement multijoueurs en ligne, souvent appelés mondes persistants ${ }^{14}$, se démarquent par la présence, dans le même espace de jeu, d'un grand nombre de joueurs. Ils mettent ainsi de l'avant la dimension sociale du jeu, et se targuent d'offrir un monde fictionnel dynamique, en constante évolution grâce à de fréquentes modifications ou extensions.

Valkyria Chronicles est à la fois jeu de stratégie et jeu de rôle. S'il place au centre de son expérience un récit complexe, il demande au joueur une réflexion stratégique, axée sur la gestion de ressources. D'un autre côté, cette œuvre particulière développe un récit riche, avec des personnages complexes, qui prend la forme de courts films d'animation.

\footnotetext{
${ }^{13} S^{\prime}$ il ne mentionne pas ce jeu spécifique (qui n'était pas paru à l'époque), Henry Jenkins offre une discussion intéressante de la notion de « bac à sable » dans son blogue : Henry Jenkins - «The future of Sandbox Games », Confessions of an Aca-Fan (10 décembre 2007). < http://henryjenkins.org/2007/12/gambit.html> (11 septembre 2011)

14 On retrouve dans l'introduction du 2004 Persistent Worlds Whitepaper une liste exhaustive de termes synonymes; le terme le plus inclusif demeure celui de « monde persistent ». IGDA Online Games SIG - 2004 Persistent Worlds Whitepaper, 2004. <http://www.igda.org/online/IGDA_PSW_Whitepaper_2004.pdf>, p.6. (11 septembre 2011)
} 
Hésitant entre son côté stratégique et son côté fictionnel, Valkyria Chronicles se rapproche énormément des jeux parfois surnommés « films interactifs »15 pour leur forte linéarité, et leur approche non interactive du développement du récit.

En plus d'être parus sensiblement au même moment, les trois jeux sélectionnés ont le mérite d'avoir été d'énormes succès critiques : le site de critiques Metacritic accorde à Fallout 3 une note moyenne de 91 (sur un total de 100), la même note à World of Warcraft : Wrath of the Lich King ainsi qu'un respectable 86 à Valkyria Chronicles. ${ }^{16}$ Les trois œuvres ont été suivies d'autres jeux dans la même lignée, et ont été honorés de différents prix et récompenses. ${ }^{17}$ Le succès tant populaire que critique de ces trois jeux fait aussi partie des raisons qui nous ont poussé à les retenir : ce ne sont pas des jeux expérimentaux, obscurs ou oubliés, et ils représentent bien, à ce titre, l'état général de l'industrie vidéoludique au moment de leur parution.

15 Travis Fahs - «The Lives and Deaths of the Interactive Movie», IGN (3 mars 2008). <http:// retro.ign.com/articles/856/856379p1.html> (11 septembre 2011)

16 «Fallout 3 », Metacritic (2011). <http://www.metacritic.com/game/pc/fallout-3> (14 septembre 2011); "World of Warcraft Wrath of the Lich King ", Metacritic (2011). <http://www.metacritic.com/game/pc/world-of-warcraft-wrath-of-the-lich-king> (14 septembre 2011); «Valkyria Chronicles», Metacritic (2011). <http://www.metacritic.com/game/playstation-3/valkyriachronicles> (14 septembre 2011).

17 Fallout 3 a été voté « Game of the Year » par les Game Developers Choice Awards, IGN et les Golden Joystick Awards: «9th Annual Game Developers Choice Awards», Gante Developers Choice Awards (2009). <http://www.gamechoiceawards.com/archive/gdca_9th.html> (14 septembre 2011); "IGN 2008 Game of the Year", IGN (16 janvier 2009). <http://video.ign.com/dor/articles/944244/ign-game-of-the-year-2008revealed/videos/igngoty2008_winner_011609.html> (14 septembre 2011); «Fallout 3 Wins The Golden Joysticks », Kotaku (30 octobre 2009). <http://kotaku.com/5393592/fallout-3-wins-the-golden-joysticks> (14 septembre 2011).

World of Warcraft: Wrath of the Lich King a été nommé « Massively Multiplayer Game of the Year» aux Interactive Achievment Awards: " 2009 Interactive Achievement Awards», Academy of Interactive Arts and Sciences (2009). <http:/ / www.interactive.org/awards/2009_12th_awards.asp> (14 septembre 2011).

Valkyria Chronicles a été honoré par les Guinness World Records en tant que « BestStrategy Game for the PS3 »: «E3 2010: Guiness World Records », SEGA Blog (17 juin 2010). <http://blogs.sega.com/2010/06/17/e3-2010guinness-world-records/> (14 septembre 2011). 
Notre analyse de ces trois jeux portera sur trois différents aspects, qui auront chacun leur chapitre, et qui sont éminemment reliés : celui de la fiction, celui du récit, et finalement, celui de la lecture. Individuellement, ces aspects ont fait couler beaucoup d'encre, et se sont retrouvés au cour de bien des débats. Nombre de chercheurs et de penseurs s'opposaient en effet à l'idée même d'approcher le jeu électronique comme une œuvre de fiction, et plus encore comme un récit. Nous pensons ici aux « ludologistes » Jesper Juul,18 Espen Aarseth ${ }^{19}$ ou Gonzalo Frasca ${ }^{20}$, qui estimaient généralement qu'une telle approche relevait d'un impérialisme théorique et qu'elle ne s'attardait ainsi pas à la nature profonde du jeu électronique: la simulation d'un système de règles. ${ }^{21}$ À l'autre extrême, on retrouvait des théoriciens comme Marie-Laure Ryan²2, Janet Murray ${ }^{23}$ ou Henry Jenkins ${ }^{24}$, qui considéraient le jeu électronique comme un nouveau mode de représentation essentiellement narratif, qui n'est donc pas fondamentalement différent des autres formes de récit. Ces penseurs se méritent le titre de «narrativistes ». Les débats se sont depuis estompés, et si chaque camp maintient généralement le cap, les deux approches sont depuis le début des années 2000 plutôt considérées comme complémentaires. ${ }^{25}$

\footnotetext{
18 Jesper Juul - A Clash between Gane and Narrative, University of Copenhagen Press, Copenhagen, 2001.

19 Espen Aarseth - Cybertext; Perspectives on Ergodic Literature, John Hopkins University Press, Baltimore, 1997.

${ }^{20}$ Gonzalo Frasca - Videogames of the Oppressed - Videogantes as a Means for Critical Thinking and Debate, Thèse de maîtrise (Information, Design and Technology), Georgia Institute of Technology, 2001. <http://www.ludology.org/articles/thesis/FrascaThesisVideogames.pdf> (14 septembre 2011)

21 C'est là la pensée développée dans Gonzalo Frasca - «Simulation versus Narrative: Introduction to Ludology » in The Video Gane Theory Reader, Routledge, New York, 2003, p.221-235.

22 Marie-Laure Ryan - «Beyond Myth and Metaphor: The Case of Narrative in Digital Media », Game Studies, Vol. 1, No. 1 (Juillet 2001). <http://www.gamestudies.org/0101/ryan/> (11 septembre 2011)

${ }^{23}$ Janet Murray - Hanlet on the Holodeck: The Future of Narrative in Cyberspace, MIT Press, Cambridge, 1998.

24 Henry Jenkins - «Game Design as a Narrative Architecture», Henry Jenkins blog. <http://web.mit.edu/cms/People/henry3/games\&narrative.html> (14 septembre 2011)

${ }^{25}$ On voit bien cette tendance dans Gonzalo Frasca - Ludologists love stories too : notes from a debate that never took place, présenté à la conférence DIGRA (2003). <http://www.ludology.org/articles/Frasca_LevelUp2003.pdf> (11 septembre 2011) et dans Jesper Juul - Half-Real: Video Games Between Real Rules and Fictional Worlds, MTT Press, Cambridge, 2005.
} 
Pour notre part, nous désirons inscrire notre démarche dans une position intermédiaire. En effet, nous considérons qu'il faut cerner la spécificité du vidéoludique, nécessairement liée à sa capacité de simulation. Toutefois, contrairement aux ludologistes, nous croyons que le fonctionnement de la fiction n'est pas foncièrement différent de celui du jeu. Pour nous, les deux obéissent à un système de règles, qui codifie ce qui est et ce qui peut être dans chacun. Nous postulons donc que le récit, au final, respecte un code établi, comme le jeu, et qu'il nous sera possible de penser les deux comme des systèmes similaires. C'est là une position qui diffère également de la pensée narrativiste, puisque nous ne considérons pas le jeu comme une forme de récit, mais plutôt les deux comme deux faces d'une même réalité structurelle. Certaines approches littéraires ${ }^{26}$ considèrent déjà la fiction, le récit et la lecture comme un réseau lié à un système de règles fictionnelles : nous proposons ainsi mettre en relief l'adéquation entre ces approches et le jeu, et nous espérons ainsi mieux faire ressortir la spécificité des œuvres vidéoludiques.

En ce qui concerne la fiction vidéoludique, nous essaierons de détailler les conditions d'émergence de celle-ci au sein de l'œuvre. Nous considérerons la fiction vidéoludique comme résultant de la rencontre entre un système de règles et de propriétés fictionnelles, et un système de règles de jeu. Notre approche s'inspirera principalement de la réflexion de Umberto Eco présentée dans Lector in Fabula ${ }^{27}$ concernant les propriétés fictionnelles. Sa façon de penser la fiction pourra éclairer la notion de fiction comme système de règles. Nous ferons aussi fortement appel aux travaux de Jesper Juul sur l'interaction entre jeu et

\footnotetext{
${ }^{26}$ En particulier, nous pensons à l'approche systémique, que nous aurons l'occasion de détailler.
}

27 Umberto Eco - Lector in fabula; Le rôle du lecteur, Éditons Grasset, Paris, 1985. 
fiction, particulièrement au très influent Half-Real. ${ }^{28}$ Juul y propose en effet la complémentarité des deux éléments pour expliquer l'unicité du vidéoludique, ce qui convient indubitablement à notre dessein.

Nous étudierons le récit à l'intérieur des jeux électroniques en nous basant principalement sur l'approche avancée par Bertrand Gervais dans son ouvrage Récits et actions. ${ }^{29}$ C'est donc dire que nous observerons les éléments constitutifs du récit, à savoir la représentation de l'action, ainsi que les modes d'enchaînement de cette dernière, pour ensuite y greffer notre conception du jeu. Nous ferons également appel aux travaux de Ryan $^{30}$ et de Fotis Jannidis ${ }^{31}$ concernant la narration vidéoludique afin de cerner les particularités propres au jeu électronique, et de pouvoir ainsi y adapter le modèle de Gervais. Jannidis, particulièrement, s'étant attardé aux enchaînements d'actions et d'intrigues dans les jeux en ligne, pourra étoffer l'approche plutôt textuelle de Gervais.

Pour ce qui est de la lecture, nous l'aborderons principalement sous l'angle des mondes possibles, établi d'abord par Eco, puis repris par Ryan. ${ }^{32}$ Cette approche nous permettra non seulement de comprendre le fonctionnement des inférences du lecteur-joueur, qui viennent motiver ses choix, mais aussi les réactions du système informatique, géré par une intelligence artificielle. Nous tenterons également de cerner les modalités de lecture

28 Jesper Juul - Half-Real: Video Games Between Real Rules and Fictional Worlds.

${ }^{29}$ Bertrand Gervais - Récits et actions. Pour une théorie de la lecture, Les Éditions du Préambule, Longueuil, 1990.

30 Marie-Laure Ryan - «Beyond Myth and Metaphor : The Case of Narrative in Digital Media ».

31 Fotis Jannidis - "Event-Sequences, Plots and Narration in Computer Games ", Dichtung Digital (2006). $<$ http://dichtung-digital.mewi.unibas.ch/2006/01/Jannidis/index.htm> (14 septembre 2011)

32 Marie-Laure Ryan - Possible Worlds, Artificial Intelligence, and Narrative Theory, Indiana University Press, Bloomington \& Indianapolis, 1991. 
propres au jeu électronique, spécifiquement concernant les différentes interactions possibles entre lecteur-joueur et œuvre, en nous inspirant de l'approche proposée par Espen Aarseth dans Cybertext concernant la littérature dite « ergodique ».33

Aborder le récit dans le cadre des jeux électroniques en mettant l'accent sur ces trois éléments nous permettra de dresser un vaste portrait des interactions entre jeu et fiction, qui donnent lieu à une nouvelle forme de récit et de lecture. En passant de la fiction au récit, et du récit à la lecture, nous faisons le choix de nous attarder d'abord au niveau macro-structurel (les systèmes de règles régissant l'expérience du jeu et de la fiction), puis au niveau micro-structurel (les enchaînements possibles des différentes actions constituant le récit) et finalement au niveau de la réception (les processus de lecture, d'interaction et de sélection des actions). Tout au long du mémoire, nous tenterons de cerner les qualités propres au jeu électronique, notamment à travers l'analyse de cas de figures, pour dresser un portrait des nouvelles possibilités qu'il peut offrir, et établir un vocabulaire pour parler de la fiction, du récit et de la lecture vidéoludiques.

${ }^{33}$ Espen Aarseth - Cybertext; Perspectives on Ergodic Literature. 


\section{CHAPITRE I}

\section{FICTION ET JEU - ENSEMBLE(S) DE RÈGLES VIDÉOLUDIQUES}

Les jeux électroniques, on l'a vu en introduction, sont considérés par Juul (et par une partie considérable du discours académique) comme porteurs d'une nature double : d'une part, ils sont les héritiers d'une tradition bien plus ancienne de jeux $x^{34}$; de l'autre, ils présentent, lorsqu'ils déploient des récits, des caractéristiques propres à la fiction. Aussi, si on souhaite tenir un discours conséquent sur cette forme de fiction (relativement) nouvelle et sur sa structure, il est impératif de ne pas oublier son aspect ludique. C'est donc dans cette optique que procédera cette première approche du médium.

Nous voulons dans ce chapitre relever la façon dont fonctionnent, en tant que systèmes structurants, la fiction et le jeu, pour ensuite faire ressortir leurs similitudes, ainsi que les contraintes, nécessités et possibilités offertes par leur coprésence. Dans cette optique, nous commencerons par établir une approche au jeu qui lui accorde une certaine fictionnalité, puis de la fiction et du jeu comme ensembles de règles. Cette démarche nous demandera également de nous positionner par rapport à la pensée académique concernant le jeu électronique. Par la suite, nous développerons sur les modalités d'interaction entre les deux ensembles (règles de la fiction et $\mathrm{du}$ jeu), ainsi qu'à propos des objectifs poursuivis par les différentes approches à leur interaction.

\footnotetext{
34 Voir Johan Huizinga - Hono ludens : Essai sur la fonction sociale du jeu ou Roger Caillois - Les Jeux et les hommes: le masque et le vertige, qui se préoccupent des jeux au sens général, bien avant l'apparition des jeux électroniques grand marché, et qui sont souvent repris par les chercheurs du domaine vidéoludique.
} 
Commençons par quelques mots sur la notion de fictionnalité. Cette dernière ne se limite pas ici à la notion de fiction comme un «type de discours intentionnellement, mais sans volonté de tromperie, non-vrai »35. C'est là une approche trop vague pour les besoins de notre réflexion. Nous proposons plutôt de considérer la fiction comme un ensemble d'éléments (que nous dénombrerons bientôt) régissant le discours fictionnel, et la fictionnalité comme la capacité à établir cette structure. Pour ce faire, nous allons emprunter à $\mathrm{Eco}^{36}$ le concept de «propriétés» des objets, des personnages et des événements, mises en scène par le texte, qui construisent l'expérience de lecture. Une fiction, dans notre réflexion, est ainsi constituée par un ensemble de propriétés qui génèrent et structurent un récit et sa lecture. Deux types de propriétés peuvent être dégagées $^{37}$ : les propriétés qui sont essentielles au monde fictionnel, qui sont statiques, et déterminent ce qui y est ou non possible (les hommes ne peuvent voler, les oiseaux oui) et celles nécessaires au récit, dynamiques, dont la variance construit celui-ci (Elizabeth Bennett déteste d'abord M. Darcy, puis apprend à $\mathrm{l}^{\prime}$ aimer $\left.{ }^{38}\right)$. Le récit devient alors une actualisation des propriétés fictionnelles, qui peuvent être inférées à partir de lui.

Si nous considérons la fiction comme un système de propriétés générant le récit, et donc l'expérience de sa lecture, de nombreux chercheurs en vidéoludique se basent sur une

\footnotetext{
35 David Herman, Manfred Jahn et Marie-Laure Ryan - The Routledge Encyclopedia of Narrative Theory, Routledge, New York, 2005, p. 163. (Nous traduisons)

36 Voir Umberto Eco - Lector in fabula; Le rôle du lecteur pour une discussion bien plus détaillée sur la notion de propriété. La vision que nous développons ici de la fiction ne correspond pas tout à fait à celle d'Eco, mais elle en est tout de même largement redevable.

${ }^{37}$ Les termes sont empruntés à Eco (dans Lector in fabula; Le rôle du lecteur).

${ }^{38}$ Nous faisons ici allusion à un célèbre roman de Jane Austen... (Jane Austen - Orgueil et Préjugés, Christian Bourgois Éditeur, Paris, 1979.)
} 
conception fort différente. Certains se limitent bien souvent à l'idée de récit, sans égard pour l'importance des conditions d'existence de celui-ci, comme s'il ne s'agissait que d'un élément greffé, sans structure pour le supporter. ${ }^{39}$ Ce faisant, ils opposent les deux, arguant que la condition d'existence du jeu est un ensemble de règles, qui ne peut supporter le récit. Toutefois, en envisageant plutôt la fiction comme structure et construction, nous supposons que la présence du récit dans un jeu électronique implique une telle structure. C'est donc dire que la nature double (jeu et récit) des jeux électroniques étudiés ici présuppose une structure elle aussi double: ils possèdent un aspect fictionnel et un aspect ludique, tous deux régis par un système de règles (les propriétés, conditions d'émergence fictionnelles, pour l'un, et les règles de jeu pour l'autre) distinct. Il serait par contre vain de vouloir analyser l'un sans traiter l'autre - ce serait mettre de côté une part essentielle de notre objet d'étude. Il faut donc approfondir le fonctionnement de ces deux structures et déterminer comment leur tension crée une expérience nouvelle, à la fois de lecteur et de joueur.

Nous avons déjà abordé les propriétés fictionnelles, aussi désirons-nous maintenant dresser un portrait de la littérature sur les règles de jeu. Pour Caillois ce sont elles qui «gouverne[nt] $»^{40}$ le jeu - et donc, en constituent la condition d'existence sine qua non. Si

${ }^{39} \mathrm{C}^{\prime}$ est d'ailleurs une erreur fréquemment commise par certains ludologistes (Espen Aarseth - Cybertext; Perspectives on Ergodic Literature et Jesper Juul - A Clash Between Game and Narrative, notamment), qui vient miner leurs conceptions. En adoptant un point de vue différent sur la fiction, la relation conflictuelle entre jeu et récit s'assouplit considérablement, comme le démontre l'évolution de la pensée de Juul jusqu'à son ouvrage majeur Half-Real: Video Games Between Real Rules and Fictional Worlds.

40 Roger Caillois - Les Jeux et les hommes : le masque et le vertige, p.11. 
certains ont tenté de penser les règles comme une série de contraintes et de limites ${ }^{41}$, la tendance actuelle se situe dans le sillage de Caillois. On peut observer cette évolution dans le panorama dressé par Juul dans Half-Real, afin de comparer diverses définitions du jeu : si toutes les approches parlent de règles, seule celle de Suits les associe à un interdit. En fait, on parle plutôt soit des règles comme d'un "système formel », soit d'objectifs et d'actions possibles "définis » par les règles.42 Il faut donc voir les règles (du jeu) non comme une série de contraintes, mais plutôt comme un ensemble de conditions qui construisent le jeu lui-même, qui le rendent possible, tout comme la fiction (qui s'apparente alors également à un ensemble de règles) rend possible le discours fictionnel, et donc le récit.

La fiction et le jeu constituent ainsi chacun un «ensemble réglé. »33 Les deux ne fonctionnent cependant pas exactement de la même façon : Archibald avance à cet effet qu'alors que la fiction propose les règles constituant son récit, le jeu, pour être perçu comme tel, doit plutôt poser les siennes, qui doivent être préalablement comprises et assimilées par le joueur. ${ }^{44}$ Cette nuance majeure, toutefois, est rapidement remise en question par le même auteur, qui avance que le support informatique permet d'éloigner le jeu électronique $\mathrm{du}$ «monde posé des jeux [pour le] rapproche[r] du monde proposé des

\footnotetext{
41 Notamment: Bernard Suits, The Grasshopper: Gantes, Life and Utopia, University of Toronto Press (1978).

${ }_{42}$ Jesper Juul - Half-Real: Video Ganes Between Real Rules and Fictional Worlds, p. 29-36.

43 Samuel Archibald - Le texte et la technique: la lecture à l'ère des nouveaux médias. Thèse de doctorat (sémiologie), Université du Québec à Montréal, 2008. <http://www.archipel.uqam.ca/902/> (14 septembre 2011), p.176.

${ }^{44}$ Nous paraphrasons ici plusieurs des idées soulevées dans Ibid., p.174-190.
} 
récits. »45 Les deux ensembles de règles se rapprochent ainsi dans le jeu électronique, si bien qu'il devient impensable de classer ses différents éléments comme appartenant soit à l'un, soit à l'autre - les éléments du jeu électroniques doivent être alors compris comme à la fois ludiques et fictionnels. Nous retenons ici l'illustration qu'en fait Archibald : le déplacement d'un cavalier, dans une partie d'échecs sur ordinateur, obéit à une règle « réelle, parce qu'elle est programmée ${ }^{46}$ mais est aussi partie prenante de la fiction, produisant « un résultat perceptible uniquement dans un monde fictionnel. ${ }^{47}$

La conception que nous venons de développer, rapprochant les structures fictionnelle et ludique en les traitant toutes deux comme des ensembles de règles qui s'entre-influencent, va en quelque sorte à contre-courant. Pour de nombreux chercheurs, la nature profonde des jeux électroniques demeure ludique, ce qui les amène à contester non pas la présence de la fiction, mais la pertinence de son analyse, considérant son statut optionnel.48 Néanmoins, plusieurs chercheurs se sont penchés sur la fiction vidéoludique, souvent à l'exclusion des autres dimensions du jeu électronique. ${ }^{49}$ La vision que nous cherchons à développer ici, même si elle se veut axée principalement sur la fiction (et plus tard, le récit et sa lecture) dans le jeu électronique, ne peut en aucun cas faire abstraction des règles de

\footnotetext{
${ }_{45}$ Ibid., p.182.

${ }^{46}$ Ibid., p.184.

47 Ibid.

48 On pense notamment aux travaux d'Aarseth dans Cybertext; Perspectives on Ergodic Literature, à ceux de Frasca pour la revue Parnasso (Gonzalo Frasca, «Ludology meets Narratology: Similitude and differences between (video)games and narrative", Parnasso, No. 3 (1999). < http://www.ludology.org/articles/ludology.htm >), ou aux premiers textes de Juul, comme A Clash Between Game and Narrative. La dominance du discours tend à s'estomper avec les années qui passent, cédant place à des approches plus nuancées dont s'inspire notre analyse.

49 C'est ce qui va mériter à Ryan, notamment, des accusations d'impérialisme théorique, pour des textes comme «Beyond Myth and Metaphor: The Case of Narrative in Digital Media ».
} 
jeu, spécialement en tant qu'ensemble structurant. C'est donc une approche similaire à celle de Juul dans Half-Real qui prévaudra : l'étude non pas de la fiction ou du jeu, mais plutôt du rapport entre les deux.

Avant tout, il convient d'explorer plus précisément ce que Juul cherche à démontrer. Héritier de la tradition académique ludologiste, il pose sans contredit les règles du jeu comme fondement du jeu. Cependant, il introduit dans sa reflexion la fiction - qui pour nous, rappelons-le, est également composée d'un ensemble de règles. Ainsi, sa pensée, dans la même optique que notre analyse, jongle avec l'idée que ces deux notions sont en fait complémentaires, et s'influencent l'une l'autre. Pour bien exprimer les modalités de cette interaction, Juul propose un modèle de classification des jeux selon l'importance accordée à leur aspect fictionnel. Voici ce modèle - qui sera légèrement remanié pour les besoins de notre travail - où Juul identifie cinq catégories principales :

1. Jeux abstraits. Un jeu abstrait est un jeu qui ne représente pas, dans son entièreté ou dans ses composantes individuelles, autre chose: le jeu de dames est un ensemble de pièces qui ne signifient rien d'autre; le jeu est les règles. [...]

2. Jeux iconiques. Un jeu iconique en est un dont les composantes individuelles ont un sens iconique : le roi de cœur dans un paquet de cartes standard suggère un roi; sa relation aux autres rois du même paquet, ou aux autres cartes de la même suite n'est pas claire. [...]

3. Jeux à monde incohérent. Un jeu à monde incohérent est un jeu avec un monde fictionnel mais où le jeu se contredit lui-même ou dont certains éléments ne peuvent être expliqués comme faisant partie du monde fictionnel. [...] Le jeu d'échecs représente un conflit entre deux sociétés en guerre, mais il n'est pas possible d'expliquer les mouvements des pièces excepté en se référant aux règles du jeu.

4. Jeux à monde cohérent. Certains jeux contiennent des mondes cohérents, où rien ne nous empêche de les imaginer dans tous les détails. La plupart des jeux d'aventure se retrouvent dans cette catégorie.

5. Jeux mis en scène. Les jeux mis en scène sont un cas particulier où un jeu abstrait ou à un quelconque degré représentationnel est joué au sein d'un monde plus 
élaboré. Il s'agit de jeux [...] où les puzzles et jeux à résoudre sont présentés comme ayant été créés par des personnages [...].50

Si de prime abord le modèle semble pertinent, nous estimons toutefois qu'il faut fondre en une seule catégorie les jeux à monde incohérent et à monde cohérent. Il s'agit en fait d'une fausse distinction: dans les deux cas, le jeu possède de forts éléments fictionnels, spécifiquement référentiels plutôt qu'iconiques. La nuance entre les deux se situe ici au niveau du traitement de la fiction, séparant les jeux qui intègrent à cette dernière l'ensemble de leur système de règles - créant ainsi l'illusion d'être régi par un seul ensemble de règles hybridant le jeu et la fiction -, de ceux qui n'en font pas l'effort. C'est une distinction superflue : il est pratiquement impensable d'intégrer toutes les règles d'un jeu à sa fiction et, surtout, il existe d'innombrables degrés entre l'intégration parfaite et la ségrégation totale. S'il est possible, en théorie, de traiter la fiction et le jeu autant comme une instance unique que comme deux instances totalement disjointes, la pratique démontre que c'est entre ces deux positions extrêmes que se situent la plupart des jeux à caractère fictionnel - et c'est aussi là que se situe le terreau le plus riche à analyser.

Nous proposons donc de mettre de côté les cas particuliers des jeux abstraits, iconiques ou mis en scène, qui, trop spécifiques, ne sont pas d'un grand intérêt pour une analyse de la fictionnalité vidéoludique. Cela nous laisse la grande catégorie des jeux à monde fictionnel, qui présentent une grande diversité d'actualisations de l'interaction dont il a été question auparavant. Dans le corpus retenu, nous chercherons donc à analyser les efforts

\footnotetext{
50 Jesper Juul - Half-Real: Video Games Between Real Rules and Fictional Worlds, p.131-132. (Nous traduisons)
} 
d'intégration (ou de ségrégation) de ces deux systèmes de règles que sont la fiction et le jeu, efforts envisagés comme une contrainte spécifique au jeu à monde fictionnel. Pour revenir à la terminologie de Juul, nous suggérons l'appellation de «cohérence fictionnelle » pour couvrir les notions d'intégration et de ségrégation des deux ensembles de règles; plus l'intégration est élaborée, plus la cohérence référentielle est atteinte.

En plus des contraintes ludiques et fictionnelles, un dernier élément demande à être identifié pour permettre l'analyse complète de la structure des jeux vidéo. On a souvent décrit le jeu comme une abstraction du réel ${ }^{51}$, redevable ainsi d'une certaine qualité « référentielle ». De nombreuses compagnies de développement vantent le « réalisme » de leurs jeux, non seulement sur le plan graphique (qui, de toute façon, relève plutôt de la fiction) mais aussi sur celui des mécanismes de jeu. On propose par exemple de vivre avec réalisme une expérience de « carrière »,52 ou encore on promeut l'exactitude d'un nouveau moteur physique.53 Les propriétés des objets et leurs comportements peuvent ainsi posséder, en plus de la cohérence fictionnelle définie plus haut, une cohérence référentielle. $C^{\prime}$ est donc à dire qu'à la relation déjà complexe entre règles et fiction, on peut ajouter un troisième joueur - le monde réel. Car, si, dans un monde fictionnel, on a tendance à faire

\footnotetext{
${ }^{51}$ Notamment Johan Huizinga - Hono ludens : Essai sur la fonction sociale du jeu.

52 Mode proposé, notamment, dans les dernières incarnations des séries de jeux NFL (EA Tiburon - NFL Head Coach 09, EA Sports, 2008) et NHL (EA Canada - NHL 10, EA Sports, 2009).

53 Un moteur physique est un programme informatique qui s'occupe de la résolution de problèmes physiques traditionnels - détection de collisions, souffles d'explosion, par exemple. Il ne s'agit pas nécessairement d'une approximation des lois de la physique réelle - Diablo III (Blizzard Entertainment - Diablo III, Blizzard Entertainment), annoncé pour 2011, ne les respecte que très vaguement.
} 
référence, sauf avis contraire, aux lois du monde réel54, la nécessité d'implanter et de programmer ces lois, ainsi que les limites de la technologie informatique, demandent certains ajustements. Les dynamiques de déplacement aquatique, par exemple, sont particulièrement difficiles à implanter, et la présence généralement succincte de plans $\mathrm{d}^{\prime}$ eau fait en sorte que beaucoup de jeux choisissent sciemment un accroc profond au réalisme en empêchant le personnage d'y entrer, voire en le tuaint au simple contact d'un plan d'eau, et bien souvent sans aucune justification fictionnelle. ${ }^{55}$

Ainsi, notre analyse de la fictionnalité dans les trois jeux électroniques à l'étude se concentrera sur deux aspects principaux. D'abord, elle portera sur les modalités selon lesquelles le jeu établit (ou refuse d'établir) une certaine cohérence fictionnelle; dit autrement, il s'agira de voir comment fiction et jeu sont intégrés ou maintenues distincts. Le même type de réflexion sera ensuite appliqué aux efforts visant (ou rejetant) une certaine cohérence référentielle. Ces deux notions de cohérence fictionnelle et de cohérence référentielle nous semblent permettre une approche de la fictionnalité spécifique au jeu électronique, puisqu'elles prennent en compte les contraintes inhérentes à sa nature de jeu, et le cas échéant, au support informatique. Plus encore, on peut voir là non seulement une série de contraintes mais aussi un système formel complexe qui permet le développement et l'apparition de nouvelles modalités fictionnelles.

54 Voir Umberto Eco - Lector in fabula; Le rôle du lecteur. L'auteur y discute de nombreux cas où le texte peut se jouer de son lecteur, qui prend généralement pour acquise la validité, dans le monde fictionnel, des lois qui régissent le monde réel.

55 Le blogueur Stephen Totilo offre une excellente discussion à propos des déplacements aquatiques dans l'entrée: Stephen Totilo - «Sink or Swim? The Game Designer's Conundrum », Kotaku (2 juillet 2009). <http:/ / kotaku.com/5306343/sink-or-swim-the-game-designers-conundrum> (15 septembre 2011) 
Avant de passer à l'analyse du corpus, il demeure un dernier point à éclaircir au sujet de la cohérence, qu'elle soit fictionnelle et référentielle. Elle n'est en effet pas un simple exercice de style : elle vise bien souvent à créer un effet d'immersion ou de présence - deux concepts semblables, mais non identiques. McMahan définit l'immersion comme un «terme métaphorique dérivé de l'expérience physique d'être submergé dans l'eau. »56 Puis, reprenant les propos de Janet Murray, elle note qu' on « recherche le même sentiment dans une expérience psychologiquement immersive $[\ldots]$ : la sensation d'être entouré par une réalité complètement différente, [...] qui capture toute notre attention [...]. $^{57}$ Il faut spécifier que l'immersion n'est pas nécessairement liée au réalisme visuel ou auditif, bien que ce dernier puisse être un atout considérable, comme on le verra ultérieurement. Pour le moment, on peut identifier trois conditions qui contribuent à créer l'effet d'immersion :

(1) Les attentes de l'usager doivent coïncider assez étroitement avec les conventions de l'environnement.

(2) Les actions de l'usager doivent avoir un impact non trivial sur l'environnement.

(3) Les conventions du monde doivent être cohérentes, même si elles ne coïncident pas avec celles $\mathrm{d} u$ « meatspace ». [Monde réel] ${ }^{58}$

Plus encore, McMahan élabore l'idée que l'immersion peut se faire de façon diégétique ou non diégétique (par l'entremise des règles de jeu) dans un processus qu'elle nomme « engagement ».59 $C^{\prime}$ est donc dire que la logique et la cohérence interne, sur le plan de la fictionnalité ou du système de règles, du monde suffisent. Une forte cohérence fictionnelle

\footnotetext{
56 Allison McMahan - «Immersion, Engagement and Presence: A Method for Analyzing 3-D Video Games » in The Video Gane Theory Reader, Routledge, New York, 2003, p.71. (Nous traduisons)

57 Janet Murray - Hanilet on the Holodeck: The Future of Narrative in Cyberspace, MIT Press, Cambridge, 1998, p.98-99.

58 Allison McMahan - «Immersion, Engagement and Presence: A Method for Analyzing 3-D Video Games », p.68-69. (Nous traduisons)

59 Ibid., p. 69.
} 
vient combiner les deux expériences d'immersion, mais n'est pas nécessaire - le joueur peut très bien vivre les deux séparément, ou seulement l'une des deux. La cohérence référentielle demeure intéressante, quant à elle, parce qu'elle rend les conventions du jeu plus intelligibles - et, donc, plus aptes à coïncider avec les attentes du joueur, conformément à la première condition.

Lorsqu'on parle d'effet de présence, par contre, le réalisme (autant sur le plan des règles que sur celui des interfaces représentationnelles) devient plus important. Le terme vient de la recherche en réalité virtuelle, et concerne le sentiment $\mathrm{d}^{\prime}$ «être là », créé par une combinaison de facteurs menant à une «illusion de non-médiation. " ${ }^{60}$ Là où l'immersion ressemble plutôt à une suspension de l'incrédulité, la présence exige qu'il n'y ait pas d'incrédulité à suspendre. Si on peut douter (à juste titre) de la «faisabilité » du concept, il désigne néanmoins un certain idéal à atteindre - du moins en réalité virtuelle. Dans l'univers des jeux électroniques, il peut être un effet recherché, mais ne constitue pas toujours l'objectif principal.61 C'est un effet de réalisme, certes; mais, il ne s'atteint pas uniquement avec les meilleurs moteurs graphiques ou logiciels auditifs - on le retrouve aussi dans les ensembles de règles, par l'intégration d'une certaine cohérence référentielle, d'où son importance ici.

Pour résumer, il sera question de deux éléments principaux dans l'étude du corpus : la cohérence fictionnelle (intégration des règles de jeu et de la fiction) et la cohérence

${ }^{60} \mathrm{Ibid} ., \mathrm{p} .73$. (Nous traduisons)

${ }^{61}$ Un exemple de jeu qui recherche l'effet de présence : les simulateurs de vol. Voir à ce sujet Jesper Juul - $A$ Clash Between Game and Narrative. 
référentielle (approximation du réel par les règles). On verra aussi comment ces éléments et leur traitement font partie d'une approche spécifique de l'immersion et de la présence : quel effet précis, par exemple, les modalités de cohérences fictionnelle et référentielle de chacun des jeux parviennent-elles (ou cherchent-elles) à créer - et pourquoi cet effet plutôt qu'un autre? On déterminera également la conception de la fiction propre aux jeux électroniques, que ces multiples contraintes et «idéaux» à atteindre contribuent à construire. Sur ce point, une avenue mérite d'être explorée : l'impact du jouẹur sur l'attribution de propriétés, dans le cadre de la fiction ou des règles. En effet, le joueur est parfois amené à contribuer en dehors même de l'expérience de jeu, afin de déterminer certaines des propriétés du monde fictionnel ou ludique, en particulier par le processus de création de personnage.

\section{Fallout 3}

Fallout 3, le premier jeu à l'étude, ne manque pas d'intérêt sur le plan de la fictionnalité. Hybridant deux familles de jeux électroniques en apparence différentes (jeux de tir à la première personne et jeux d'aventure), et descendant direct des jeux de rôle à la première personne ${ }^{62}$, il emploie diverses stratégies de cohérence propres à l'une ou l'autre - voire en développe de nouvelles.

\footnotetext{
${ }^{62}$ En particulier, on pense ici aux jeux plus anciens de Bethesda Softworks, spécialement The Elder Scrolls III : Morrowind (Bethesda Softworks - The Elder Scrolls III: Morrowind, Bethesda Softworks, 2002) et The Elder Scrolls IV : Oblivion (Bethesda Softworks - The Elder Scrolls IV: Oblivion, 2K Games, 2006).

De nombreux critiques ont décrit Fallout 3 comme «Oblivion with guns », alludant à sa similitude avec le jeu d'aventure déjà mentionné Oblivion avec pour particularité la présence d'armes à feu. En particulier, nous pensons à Joakim Bennett dans l'édition suédoise du magazine PC Gamter, dans son numéro d'octobre 2008, cité par Kevin Spiess - "Swedish reviewer: Fallout 3 "Oblivion with guns", gives game a. 81\% 》, Neoseeker (8 octobre 2008). <http://www.neoseeker.com/news/8987-swedish-reviewer-fallout-3-oblivion-with-gunsgives-game-a-81/> (15 septembre 2011)
} 
Si on passe outre le menu du jeu, qui fait office de page couverture, et qu'on débute une nouvelle partie, on constate rapidement que Fallout 3 multiplie les efforts pour déguiser ses règles de jeu. Le processus de création de personnage, qui auparavant ${ }^{63}$ faisait partie d'un processus préparatoire, préambule au jeu lui-même, non seulement fait maintenant partie du jeu mais est intégré de façon exemplaire à sa fiction. On commence à la naissance du personnage principal et, afin de laisser au joueur le soin d'en déterminer l'apparence physique ( $\mathrm{du}$ sexe à la forme de l'arcade sourcilière), on passe par un ordinateur surpuissant capable d'anticiper son évolution, qui est présenté au père (ce qui explique le changement d'interface graphique). On inscrit et justifie ainsi au sein de la fiction un processus qui n'en fait que rarement partie. Il en va de même pour déterminer le nom du personnage : c'est à travers la bouche du père qu'on laisse le choix au joueur.

On saute quelques années, et le personnage contrôlé par le joueur est maintenant un bébé un peu casse-cou. Il apprend tout juste à marcher : voilà qui justifie bien la présentation des moyens d'interaction de base, à savoir avancer, courir, sauter. Chemin faisant, on trouve un livre éducatif au sol :

63 Voir Black Isle Studios - Fallout, Interplay, 1997 et Black Isle Studios - Fallout 2, Interplay, 1998. 

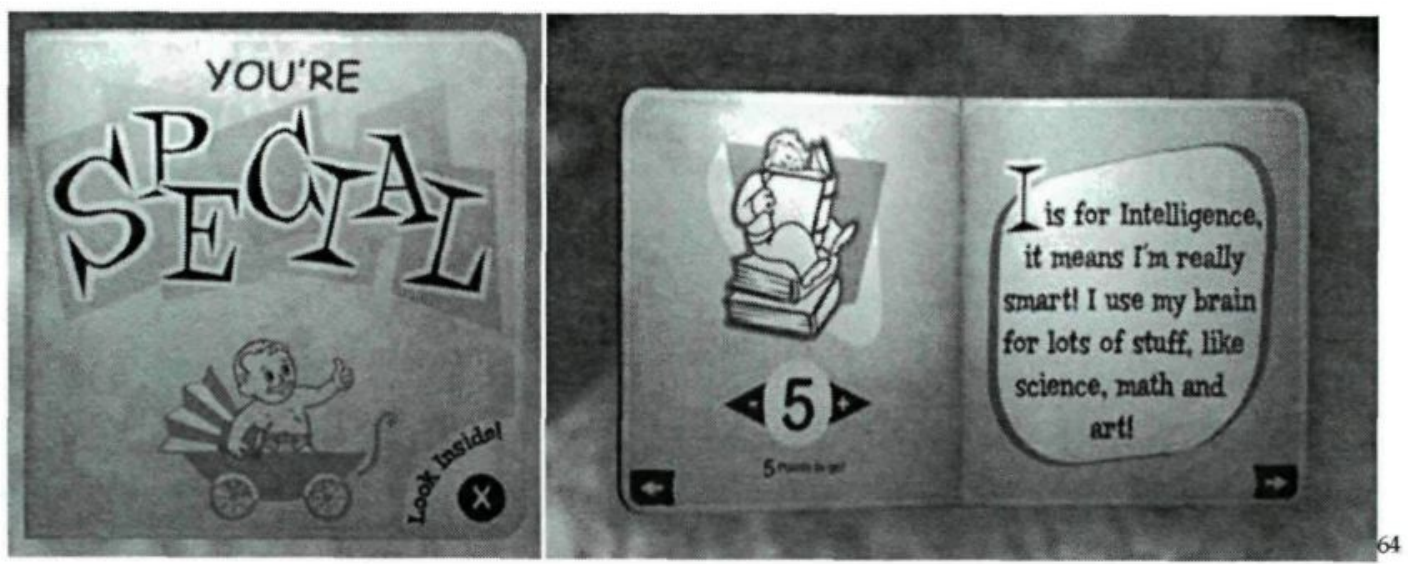

On demande au joueur de déterminer les statistiques ${ }^{65}$ de son personnage: Strength, Perception, Endurance, Charisma, Intelligence, Agility et Luck. Chaque page (comme celle reproduite ci-haut) est consacrée à l'une d'entre elles, la détaillant avec un langage enfantin. Le processus, encore une fois, est intégré à la fiction. Ce n'est d'ailleurs pas terminé : préadolescent, le personnage célèbre son anniversaire et la façon dont il traite les invités détermine son « karma »66. Il reçoit en cadeau une arme à feu, prétexte à lui apprendre (et par la bande, apprendre au joueur) comment s'en servir. Devenu adolescent, il doit remplir un questionnaire qui détermine ses aptitudes. Sur l'éventail de compétences qu'il est possible de développer, le jeu (se basant sur les réponses du joueur) en choisit trois qui bénéficient d'un bonus de départ. Un joueur déçu du résultat obtenu peut même convaincre l'examinateur de le falsifier, reprenant en main le processus de création tout en préservant l'effet d'immersion.

\footnotetext{
${ }^{64}$ Bethesda Game Studios - Fallout 3. (Vault 101 - Baby Steps)

${ }^{65}$ Chacune représente une caractéristique innée du personnage (par opposition aux compétences, qui sont plutôt acquises), variant de sa force physique à son charisme naturel. Bien qu'elles puissent être modifiées, les statistiques sont plutôt fixes, représentant un peu le talent brut du personnage.

${ }^{66}$ Statistique qui mesure où se situe le personnage sur l'échelle du bien et du maI.
} 
Bien que quelques propriétés attribuées au personnage principal soient fixées à l'avance (il est toujours le fils du docteur James et habite une voûte sécurisée, par exemple), de nombreuses autres, qui conditionnent autant l'expérience narrative que l'expérience de jeu, sont donc laissées à la discrétion du joueur. Si certaines, comme l'apparence physique, sont d'ordre purement esthétique, d'autres, comme les caractéristiques SPECIAL ou les compétences, peuvent limiter ou étendre les modes possibles de résolution des problèmes présentés par le jeu. Il est impressionnant de constater que l'entièreté du processus de création de personnage fait partie du jeu lui-même, qui refuse autant que possible de briser le quatrième mur. Il le refuse tellement, en fait, que même le menu du jeu - où l'on peut consulter les statistiques du personnage, mesurer son état de santé ou consulter les cartes des lieux explorés (dressées automatiquement) - est représenté par une montre ultrafuturiste que possède le personnage. Plus qu'une simple contrainte du jeu, le menu devient donc partie intégrante de son univers fictionnel : une fois le menu ouvert, on peut même aisément remarquer le bras du personnage et les contours de la montre, qui forment le cadre du menu.

Après ce processus de conception, le personnage s'enfuit et se lance à la découverte du monde qui l'entoure. Ce monde est constitué de propriétés relevant autant du domaine de la fiction que de celui des règles de jeu - et, comme on pourra le constater, l'intégration des deux est une caractéristique importante de l'univers de Fallout 3. On parle ici d'un univers fictionnel post-apocalyptique, représentant une réalité alternative : l'action se déroule quelque 300 ans après un conflit nucléaire entre les États-Unis et la Russie qui a 
changé la face de la planète. Le monde est irradié : c'est là une réalité d'ordre fictionnel, mais que communiquent également certains aspects des mécanismes de jeu. Chaque fois que le personnage est exposé à des radiations (en buvant de l'eau contaminée, par exemple), il accumule des «points" de radiation. Passé un certain degré, il souffre d'intoxication, perdant en force, en endurance ou en agilité. S'il persiste à s'irradier, il peut même en mourir.

Les mécanismes gérant l'irradiation sont composés d'un amalgame intéressant de règles de jeu et de propriétés fictionnelles : on limite les ressources du personnage, et consommer de la nourriture contaminée devient ainsi un choix complexe, le joueur devant évaluer les bénéfices et les risques d'une telle action. Simultanément, cette limitation s'inscrit dans le cadre de l'univers fictionnel nucléarisé. Cette gestion particulière des contraintes ludiques et fictionnelles est l'un des nombreux efforts de cohérence fictionnelle présents dans le jeu; il peut même être perçu comme cohérent sur le plan référentiel, rappelant des effets réels de radiations nucléaires.

En poursuivant sur le thème de la cohérence fictionnelle, on peut noter que le système de combat VATS67, qui donne au joueur un avantage certain sur ses opposants, n'est pas qu'un paramètre de jeu : il est justifié par la montre ultrafuturiste déjà mentionnée. En laissant au joueur le temps de penser ses actions, cet ajout rapproche Fallout 3 d'un jeu de

\footnotetext{
67 Vault-Tec Assisted Targetting System. Le système met le jeu en arrêt et laisse au joueur le temps de choisir quel adversaire cibler, améliorant ainsi ses chances de faire mouche. Cela symbolise l'avantage certain que donne le gadget technologique au personnage en situation de combat.
} 
rôle ou de stratégie - aussi est-il important pour le système de règles de jeu -; d'un autre côté, il fait aussi partie de l'univers fictionnel et y trouve sa justification. Cette montre ne possède pas de référent dans le monde réel - mais l'imaginaire de Fallout 3 , inspiré de la science-fiction américaine des années 1950, peut se permettre d'inventer ses propres référents.

Si la cohérence fictionnelle est fortement mise de l'avant dans Fallout 3, la cohérence référentielle n'est pas en reste pour autant. On l'a vu, déjà, avec les mécanismes d'irradiation : les deux cohérences peuvent fonctionner ensemble, et le font bien souvent. D’autres éléments font écho à une certaine expérience du réel : par exemple, l'usage de psychotropes ou d'alcool peut causer la dépendance, entraînant même des effets de sevrage. De plus, l'alcool rend plus charmant et fort, mais moins intelligent - une assez bonne approximation des effets de désinhibition et de détérioration du jugement. Par contre, il est impossible d'en consommer au point de tomber en coma éthylique, preuve que le système n'est pas parfaitement réaliste!

De nombreux autres aspects du jeu se veulent une intégration des propriétés du monde réel. Par exemple, le personnage doit reprendre son souffle à intervalles réguliers s'il plonge sous l'eau. Ou encore, on constate que chaque objet possède un poids, et que le poids transportable par le personnage est limité par sa force. L'effet d'immersion psychologique peut donc être conservé de diverses façons par le jeu, au moyen de 
l'établissement de règles qui coüncident avec les attentes du joueur, construites par son expérience du réel.

Cela dit, le système formel de Fallout 3 n'est pas toujours composé en entretenant une cohérence absolue entre fiction, règles et référents. Par exemple, le fait de manger et de boire soigne les blessures, ce qui est cohérent à l'intérieur du monde fictionnel, mais aberrant par rapport au monde réel. Le personnage peut également passer des semaines sans dormir, boire ou manger, peu importe son endurance, et l'évolution de ses capacités n'obéit enfin à aucune logique fictionnelle. Ainsi, s'il est cohérent, d'un point de vue référentiel, de s'améliorer alors qu'on gagne en expérience, la façon dont procède le jeu permet, par exemple, à un personnage qui n'a jamais tenu une arme à feu de devenir un tireur d'élite instantanément. D'un côté, il est intéressant de voir que, à l'instar du processus de création du personnage, l'évolution de ses différentes propriétés est largement laissée entre les mains du joueur; d'un autre côté, le manque de logique référentielle est flagrant.

De façon plus marquante encore, Fallout 3 présente de nombreuses incohérences référentielles par souci d'équilibre de l'expérience de jeu. Les armes à feu fonctionnent sous l'eau, par exemple, et un tir de fusil de chasse dans la tête, à bout portant, ne tue que très rarement un adversaire sur le coup. Afin de poser un défi grandissant au joueur, il est crucial que les ennemis puissent survivre à une force de frappe de plus en plus grande; à cet égard, la mesure de leur capacité à survivre en « points de vie » est une simplification 
tolérable, qui a fait ses preuves dans de nombreux jeux de rôle, et permet une plus grande latitude que ne le permettrait une référence stricte au monde réel. Du moins, l'univers du jeu demeure cohérent, car la survie du personnage se mesure elle aussi en «points de vie ", et l'effet d'immersion n'est donc que très peu menacé. Toutefois, les arrêts de jeu, la possibilité de voir au-dessus du personnage plutôt qu'à travers ses yeux, et de nombreux autres accrocs légers minent un potentiel effet de présence. Si Fallout 3 s'en rapproche plus que de nombreux autres jeux de rôle, de par son inspiration des jeux de tir, il refuse d'en faire un idéal à atteindre aux dépens d'une meilleure expérience de jeu.

L'évolution de la série des Fallout montre que la tendance se maintient. Fallout : New Vegas, le successeur de Fallout 3, reprend par exemple le même idéal de création de personnage : on remplace la naissance par une amnésie totale, l'ordinateur par un miroir, le livre par un examen médical et le test par une discussion avec le médecin, mais l'esprit est le même. Cependant, par souci de cohérence référentielle, on y offre un mode de jeu dit « hardcore » qui ajoute de nombreux éléments logiques (par rapport au monde réel) initialement omis pour simplifier l'expérience de jeu. Il devient notamment impossible de soigner un membre blessé simplement en dormant, les balles de fusil se voient attribuer un poids, et le personnage doit manger, dormir et boire à intervalles réguliers, sous peine de s'affaiblir, voire de mourir. Si les mécanismes de base du jeu demeurent généralement les mêmes, on note malgré tout que le côté immersif de Fallout 3 et les nombreux détours qu'il emprunte pour éviter de rompre la suspension de l'incrédulité du joueur se poursuivent dans Newv Vegas, et se voient même bonifiés par ce nouveau mode de jeu. 
Plus encore, l'impact non trivial des actions de l'usager sur son environnement, défini par McMahan comme une condition d'immersion, prend de plus en plus de place. Si dans Fallout 3 on permet au personnage de sauver le monde, d'éradiquer une ville complètement ou encore de retrouver un enfant disparu, il est rare que l'impact de ces actions se fasse sentir en dehors de la zone géographique concernée par elles. Fallout : New Vegas présente quant à lui de nombreuses voies d'action qui sont commentées par les animateurs de radio du monde, et mesure la célébrité du personnage pour déterminer $l^{\prime}$ impact de ses gestes pour tout le pays. Cette tendance déjà présente dans Fallout 3 est donc simplement dávantage exploitée.

En bref, on peut dire des Fallout qu'ils visent une immersion psychologique aussi efficace que possible. Même dans leurs accrocs, qui sont d'ordre référentiel plus souvent que fictionnel, ces jeux maintiennent une cohérence interne, refusant d'imposer des limites (par les règles) à la liberté du joueur.

\section{World of Warcraft : Wrath of the Lich King}

Si Fallout 3 et autres jeux à la première personne donnent dans la liberté de choix et l'immersion psychologique, on ne peut pas toujours en dire autant de $W o W$. Bien plus populaire que n'importe quel jeu en ligne l'ayant précédé68, il doit en grande partie son

\footnotetext{
68 Alors qu'en 2008 le deuxième plus populaire jeu massivement multijoueurs en ligne n'avait jamais dépassé les 3.5 millions d'abonnés, WoW pouvait se targuer d'en avoir plus de 10 millions. Bruce Woodcock - « MMOG Active Suscriptions 200,000+ 》, MMOGCHART.COM (2008). <http://www.mmogchart.com/Chart1.html> (15 septembre 2011)
} 
succès à l'attrait exercé sur les joueurs dits " occasionnels » ${ }^{69}$, qui ne recherchent pas la même expérience d'immersion. Pour l'essentiel, on peut dire de $W o W$ qu'il a dissocié immersion diégétique et non diégétique pour en faire deux expériences différentes, qui visent chacune leur clientèle. Au final, l'effet est que la fictionnalité et le système de règles ont chacun leur cohérence interne, mais ne s'influencent que très peu.

Commençons par examiner le processus de création de personnage, qui présente un intérêt certain du point de vue de la fictionnalité. Par rapport au processus intégré et complexe vu précédemment, celui de World of Warcraft pourra paraître simpliste. Pourtant, il présente un intérêt similaire, dans la mesure où il permet au joueur d'établir certaines des propriétés du personnage qu'il sera amené à incarner à l'intérieur de l'univers de jeu. L'essentiel du procédé, par opposition à Fallout, se situe en dehors du jeu : il est présenté comme un préambule, à l'instar de la description du personnage principal sur la quatrième de couverture d'un roman d'aventures. L'écran de création de personnage ressemble à ceci :

69 Pour une plus ample discussion sur les joueurs occasionnels, voir : Jesper Juul - A Casual Revolution: Reinventing Video Games and Their Players, MTT Press, Cambridge, 2009. 


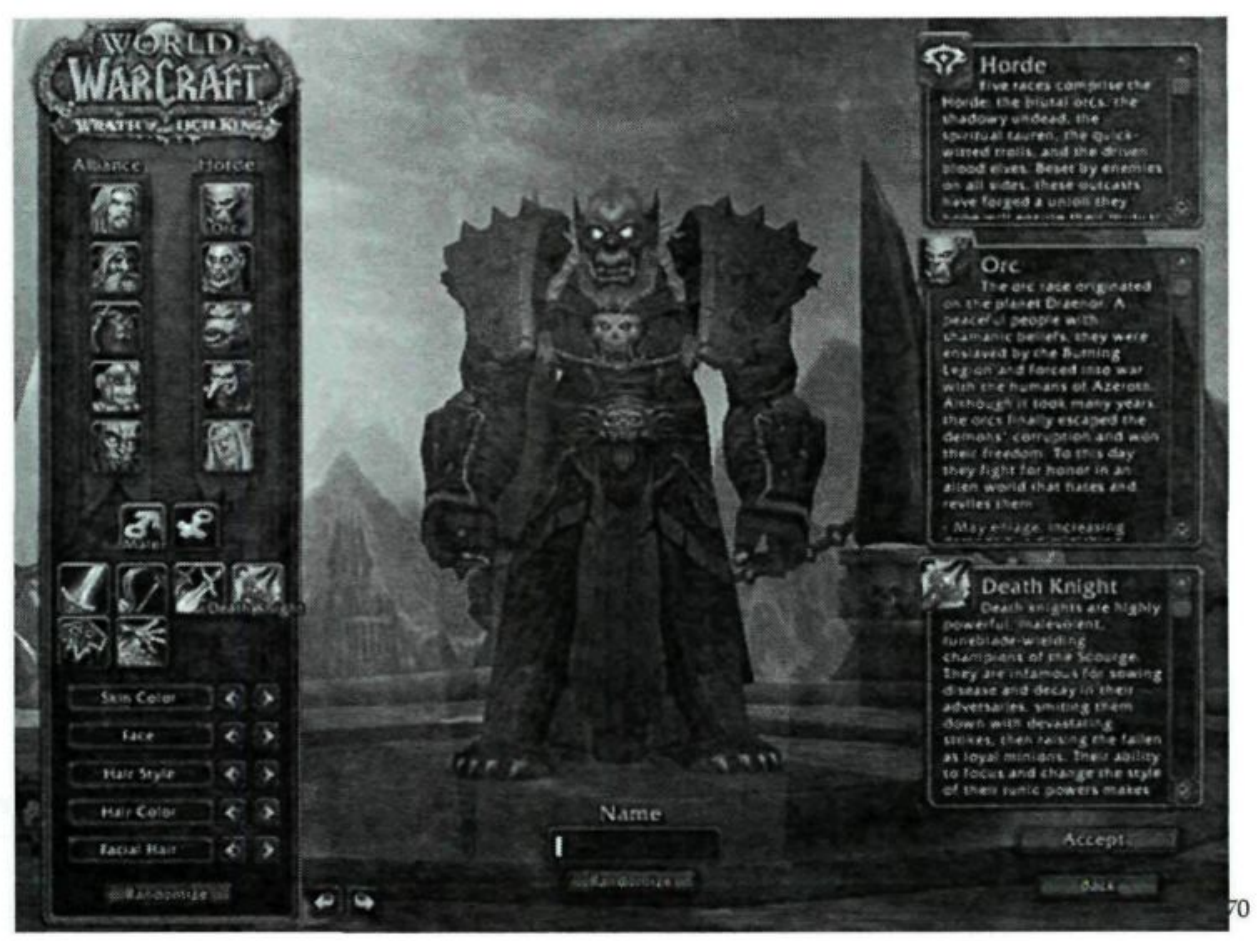

Établir les propriétés du personnage obéit à un principe simple: on choisit ses caractéristiques non pas séparément, mais comme un ensemble. À gauche, on retrouve (de haut en bas) le choix de la race, de la classe, et de l'apparence physique. Les encadrés de droite fournissent une description sommaire des choix. Comme chacun des aspects à déterminer entraîne un impact bien différent pour le jeu et sa fiction, nous proposons de les examiner à tour de rôle.

D'abord, la race: World of Warcraft offre douze choix de races plus ou moins fantastiques, qui vont du simple humain à l'obscur draenei ${ }^{71}$. Chaque race fait obligatoirement partie de l'une de deux factions en guerre : l'alliance ou la horde. Déjà, l'appartenance à l'une ou à l'autre détermine l'ensemble de quêtes auquel le joueur aura accès - un choix d'ordre

\footnotetext{
${ }^{70}$ Blizzard Entertainment - World of Warcraft: Wrath of the Lich King .

${ }^{71}$ Démons repentis hautement avancés d'un point de vue technologique.
} 
fictionnel. La race spécifique attribue des propriétés mineures au personnage (d'ordre esthétique, surtout, mais aussi sur le plan des règles du jeu) et détermine l'accès à une courte série de quêtes d'introduction. Le terme essentiel ici est mineures : pour l'expérience de jeu, le choix de la race a peu de conséquences. Toutefois, d'un point de vue fictionnel, il a un grand impact sur les motivations du personnage, qui, du reste, sont inférées par le joueur bien plus qu'établies par la progression narrative.

Le choix de la classe, lui, est d'une nature tout autre : peu important pour la fictionnalité un personnage qui lance des éclairs est-il, au final, si différent d'un autre qui lance des boules de feu? - mais hautement important pour le système de règles. Avec chaque classe vient une série de pouvoirs attribués au personnage à mesure qu'il gagne en expérience, et

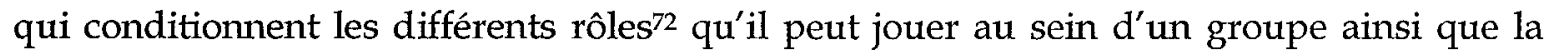
façon dont ceux-ci seront exercés. Il faut noter que le choix de la classe détermine à l'avance l'essentiel de l'évolution des propriétés attribuées au personnage : on évite ainsi l'accroc logique du libre développement présent dans Fallout 3, et on limite par le fait même le nombre de possibilités - tous les guerriers, par exemple, ont accès (avec, comme on le verra, quelques variations mineures selon la race et le choix de professions) aux mêmes pouvoirs, partageant donc un même ensemble de propriétés, sur le plan des règles de jeu.

72 Le site officiel du jeu offre une description de ces différents rôles et des classes qui peuvent les exercer. Pour plus d'information, on peut donc visiter: Blizzard Entertainment, «Classes», World of Warcraft, Cataclysm, (2011). <http:// us.battle.net/wow/en/game/class/> (15 septembre 2011) 
L'apparence physique, quant à elle, n'a aucun impact sur la structure du jeu. Homme ou femme, beau ou laid, chacun vit la même expérience ludique ou fictionnelle. On respecte ainsi la recommandation du concepteur Josh Sawyer sur le plan de l'apparence : «Si tu n'as aucune bonne raison de restreindre [les choix], ne le fais pas. $\gg^{73}$

L'essentiel des propriétés de départ, et une bonne partie de celles qui seront acquises au cours du jeu, sont donc déterminées avant le jeu lui-même, et réunies en divers ensembles ( $I^{\prime}$ ensemble race-humain, ou classe-démoniste, par exemple) entre lesquels le joueur doit choisir. Certains aspects de la progression des capacités du personnage restent à déterminer au cours $\mathrm{du}$ jeu, toutefois : son niveau de compétence en cuisine, travail du cuir ou autres professions. Celles-ci ont un impact mineur autant pour l'aspect fictionnel que pour le système de règles : certes, il y a une différence marquée entre un joaillier et un forgeron, mais à l'intérieur du jeu, rares sont les occasions où cela a un réel impact. Cependant, chaque personnage étant limité à deux professions principales, le choix de ces dernières et leur niveau de progression peuvent être déterminants sur l'immersion d'un point de vue social : cette dernière implique en effet un certain échange entre joueurs pour pallier les lacunes de chacun. Le choix des professions n'est, à ce titre, pas très différent des restrictions de rôle $\mathrm{du}$ personnage. Alors que ce dernier progresse, il acquiert des « points » de spécialisation qui lui permettent d'établir sa compétence dans un rôle bien précis. Ce choix de propriétés ludiques force le contact entre joueurs pour accomplir des efforts de groupe, qui nécessitent une bonne diversité de rôles. Ainsi, ces différents

73 Josh Sawyer - «I thought I could organize freedom. How Scandinavian of me », RPG Codex, (16 décembre 2002). <http://www.rpgcodex.net/content.php?id=24> (14 septembre 2011) (Nous traduisons) 
mécanismes de progression et d'attribution de propriétés encouragent ce que nous avons nommé précédemment l'immersion sociale - créée non par la cohérence entre systèmes ou par la consistance interne de la fiction ou des règles, mais par la qualité des échanges sociaux. McMahan voit celle-ci comme un facteur d'immersion ou de présence; en un sens, donc, l'immersion sociale ainsi créée vient compléter l'immersion diégétique et non diégétique. ${ }^{74}$

Il convient maintenant de revenir à ces deux types d'immersion. Nous avons mentionné précédemment que WoW ne faisait que très peu d'efforts en frais de cohérence fictionnelle (ce qui ne nuit pas nécessairement au processus d'immersion); un constat similaire s'applique à la cohérence référentielle. On peut même voir, à travers l'évolution de divers systèmes de jeu, comment WoW s'est distancié, avec les années, d'un modèle d'intégration par souci de simplification et d'accessibilité. On peut citer, à cet égard, le fonctionnement des « instances »75 et de la formation d'un groupe pour s'y attaquer.

À la sortie du jeu (en novembre 2004), on ne pouvait communiquer qu'avec les personnages se trouvant dans la même zone générale, ce qui demeure relativement (par rapport aux versions suivantes) cohérent d'un point de vue référentiel. Ainsi, les groupes se formaient généralement dans un grand centre de transit (villes principales) pour ensuite se mettre en route en direction du donjon. Les limites du système se sont rapidement fait sentir : certains donjons, difficiles d'accès ou situés loin des grandes villes, n'étaient que

\footnotetext{
${ }^{74}$ Voir Allison McMahan - «Immersion, Engagement and Presence: A Method for Analyzing 3-D Video Games ».

75 Sous-zone conçue pour un effort de groupe dont l'accès est interdit aux autres joueurs et qui garantit une expérience confinée et exempte d'interventions extérieures.
} 
très rarement explorés. Alors le système a évolué : les auberges du monde fictionnel se sont mises à offrir un service de « réseautage » : les personnages pouvaient y offrir leurs services, et les groupes se formaient alors automatiquement. Pour simplifier le voyage, on a ajouté des pierres de rencontre à l'entrée des donjons: on pouvait y pratiquer un rituel magique qui transportait instantanément le reste du groupe. Jusqu'à ce point, on peut dire que la cohérence fictionnelle est respectée : le système de jumelage des joueurs comme celui de transport instantané sont justifiés et expliqués par la fiction.

La version 3.3 de World of Warcraft: Wrath of the Lich King est venue redéfinir complètement ce système. Finie la cohérence fictionnelle : désormais, les joueurs peuvent se rendre disponibles pour tout donjon approprié pour leur niveau, à l'aide d'un onglet du menu. Il suffit d'y annoncer le rôle qu'on est prêt à tenir au sein du groupe, et le système de jumelage forme un groupe parfaitement équilibré, capable de faire face aux défis qui vont l'attendre. Surtout, une fois le groupe formé, les personnages en faisant partie sont transportés directement à l'entrée du donjon. Très efficace d'un point de vue fonctionnel, ce nouveau système n'essaie même pas de s'intégrer à la fictionnalité - il ne s'explique que par référence aux règles du jeu.

On peut aussi mentionner plusieurs mécanismes de jeu qui ont évolué en rejetant peu à peu la cohérence référentielle. Ainsi, afin de simuler l'apprentissage du maniement des armes, les personnages devaient, dans WoW: WoTLK, suivre un entraînement sous la direction d'un maître. Plus encore, on mesurait leur aptitude dans différents types 
d'armes (haches, épées ou arcs, par exemple) par une statistique qui ne pouvait s'améliorer qu'avec la pratique. Punitif pour les classes de personnages amenées à employer une grande diversité d'armes, le mécanisme a été retiré complètement à l'arrivée de la nouvelle expansion, Cataclysm. La classe détermine désormais les types d'armes utilisables, et l'aptitude du personnage est toujours équivalente à son niveau de pouvoir. Prenons d'autres exemples d'abandon de la cohérence référentielle. Passer trop de temps sous l'eau finissait par mener à la noyade; or, la durée d'apnée permise est passée d'une à trois minutes. L'une des races est composée entièrement de morts-vivants qui n'ont aucunement besoin de respirer - mais par souci d'équilibre, ils sont tout autant sujets à la noyade (quoiqu'après neuf minutes). Les minuscules gnomes peuvent porter les mêmes armes et armures que les immenses trolls. Enfin, il existe maintenant des objets liés au compte du joueur, que peuvent donc s'échanger librement les différents personnages du même joueur, mais qu'aucune autre personne ne peut utiliser.

Bien évidemment, certains éléments du jeu demeurent intégrés de façon efficace. On pense notamment à la façon dont le jeu gère le « respawn $»^{76}$ des personnages morts : leur âme est transportée à un esprit guérisseur, qui les somme de rejoindre leur corps pour revenir dans le monde des vivants. L'existence de la magie forme évidemment une condition de base ici pour accepter ce type de cohérence fictionnelle, ce qui ne remet pas du tout en question la cohérence interne du système. Si le personnage refuse de rejoindre son corps, il peut demander à être ressuscité par l'esprit, au cọur du cimetière le plus

\footnotetext{
76 Possibilité de retour au jeu d'un personnage décédé. Permet d'éviter de faire de la mort un terme, pour la transformer en maigre ralentissement.
} 
proche. Là, par contre, rien n'explique (d'un point de vue fictionnel) que toutes les possessions $\mathrm{du}$ personnage soient instantanément transportées sur place, et endommagées par le processus. Comme tous les accrocs conscients à la cohérence, référentielle ou fictionnelle, mentionnés précédemment, il s'agit d'un souci d'équilibre entre les joueurs on se contente de pénaliser la mort par des soucis pécuniaires (il faut réparer les objets endommagés) ou par une perte de temps (pour rejoindre son corps inanimé).

On l'a dit plus tôt, l'expérience narrative et l'expérience de jeu, bien qu'elles soient souvent dissociées, demeurent généralement consistantes - surtout celle du jeu, qui se veut équilibrée pour tous. Toutefois, pour permettre à chaque joueur de vivre la même expérience narrative, WoW empêche bien souvent un véritable impact non trivial sur l'environnement, ce qui nuit à une authentique immersion diégétique. Ainsi, malgré qu'un groupe tue la reine Onyxia, elle demeure bien portante dans la salle $\mathrm{du}$ trône, prête à être assassinée par les prochains aventuriers. Voilà qui abat définitivement le quatrième mur. Cependant, l'introduction récente de la technique dite du «phasage » permet de récupérer une certaine immersion de ce point de vue: plusieurs versions du monde existent en parallèle, et ce sont les actions passées du personnage qui déterminent dans quelle version il se trouve actuellement. Pour reprendre l'exemple de la reine Onyxia, un joueur ne l'ayant pas tuée se trouve en phase pré-assassinat, et peut lui parler dans la salle du trône. Aussitôt qu'elle est morte, il tombe irrémédiablement dans la phase postassassinat, et c'est sa successeure qui prend sa place. Le système n'est pas parfait - rien n'explique qu'un personnage puisse visiter des centaines de fois ( $s^{\prime}$ il le désire) le même 
donjon et y abattre les mêmes ennemis - mais il montre que WoW se soucie de créer un effet d'immersion: d'abord par les règles, qui évoluent de leur côté, mais aussi par l'interaction sociale et, finalement, par la diégèse, lorsque cela s'avère possible.

$C^{\prime}$ est donc dire en résumé que, pour préserver une expérience consistante d'un joueur à l'autre, WoW est prêt à rejeter cohérence fictionnelle et cohérence référentielle. L'essentiel demeure encore l'immersion, peu importe la façon dont elle se produit; elle ne cherche toutefois pas à se réaliser en intégrant autant que possible les divers systèmes structurants. Comme on le voit en regardant l'évolution du jeu, cela permet de modifier rapidement tout système jugé inefficace sans avoir à récrire nécessairement l'entier de la fiction du jeu, ni même à rechercher un « réalisme » bien arbitraire.

\section{Valkyria Chronicles}

On a vu, avec Fallout 3 et World of Warcraft: Wrath of the Lich King, deux approches très différentes du point de vue de la cohérence fictionnelle et référentielle. L'objectif visé était le même, mais la nature dynamique de WoW l'a mené à dissocier ses différentes instances structurelles pour mieux les adapter à une clientèle changeante. Valkyria Chronicles, quant à lui, se préoccupe très peu de ce type de cohérence, préférant plutôt une approche «classique $»^{77}$ de dissociation des aspects du jeu. Au demeurant, les critiques hautement favorables du jeu montrent bien qu'il n'est pas nécessaire de verser dans l'intégration

77 Particulièrement, celle favorisée par Juul dans A Clash Between Gane and Narrative. 
complexe des règles de jeu et de la fiction pour faire un bon jeu ou une bonne fiction voire les deux en même temps. ${ }^{78}$

Pour ce qui est du processus de création de personnage, Valkyria Chronicles est un cas particulier dans notre corpus : il n'en possède pas. En d'autres mots, le joueur n'a aucune latitude dans la détermination des propriétés du monde ou de ses acteurs. Cela n'est pas une mauvaise chose en soi, cependant. Les concepteurs du jeu peuvent ainsi contrôler totalement le rythme et l'efficacité de la fiction. Les propriétés étant fixes, cela assure les mêmes conditions de jeu et de lecture à chaque usager. En un sens, c'est une manière de faire qui rappelle celle de médias narratifs plus établis, comme le roman ou le cinéma.

Valkyria Chronicles laisse tout de même quelque latitude au joueur pour déterminer la progression de certaines propriétés. Chaque personnage contrôlé au cours de la partie appartient à l'une de cinq classes (scout, shock trooper, lancer, sniper, engineer) qui déterminent ses aptitudes par rapport au système de règles, et son rôle au sein de l'unité militaire commandée par le personnage principal. Il est possible d'apporter, en cours de partie, certaines améliorations à l'équipement des différentes classes, ou encore de leur fournir un entraînement plus poussé. Il faut spécifier ici que, dans les deux cas, les propriétés fictionnelles ne sónt aucunement affectées - seules les aptitudes telles que définies par les règles le sont. C'est-à-dire que peu importe la valeur numérique associée à la

${ }^{78}$ Le site de compilation de critiques Metacritic lui octroie la note très élevée de $86 \%$. On peut voir dans les commentaires de nombreuses allusions à un récit charmant et bien exécuté, ainsi qu'à des mécanismes de jeu extraordinaires. Plusieurs le qualifient même d'incontournable. «Valkyria Chronicles», Metacritic. <http:/ /www.metacritic.com/game/ playstation-3/valkyria-chronicles> (14 septembre 2011) 
puissance des personnages (leur niveau d'expérience, les dégâts infligés par leur arme) ceux-ci sont représentés dans la fiction de la même façon - le récit ne prend pas en compte cet élément. Comme certains choix doivent être faits dans ce développement (armes plus puissantes ou plus précises, par exemple), le joueur peut personnaliser l'acquisition de nouvelles aptitudes de combat ou d'équipement. En outre, les personnages sont définis, d'une façon intégrant fiction et règles, par une série de traits particuliers. Quelques-uns ne se révèlent qu'après un certain temps, si le personnage est utilisé en situation de combat. Ainsi, le joueur a un certain contrôle sur le rythme des révélations les concernant, mais pas sur la nature de celles-ci.

La plupart des traits ne sont pas conséquents d'un point de vue fictionnel, même s'ils peuvent le sembler: ainsi, les allergies au pollen ou au sable, par exemple, pourraient passer pour de la caractérisation, mais la seule mention à ceux-ci faite par le jeu est liée au système de règles.79 Certains de ces traits, et les plus importants, sont toutefois intégrés avec brio à la fiction. On pense aux différents traits d'Alicia (un des personnages principaux) qui sont fortement liés à sa nature profonde (elle est une arme de guerre créée artificiellement, ce qu'elle ignore), invoquée à plusieurs reprises par la narration. Ou plus marquant encore, le trait «Darcsen Hater »80 de Rosie, qui fait écho à son profond racisme, et qui se transforme après qu'elle ait amendé son point de vue - la fiction influençant ici les règles.

\footnotetext{
79 Les allergies réduisent les capacités des personnages qui en souffrent lorsqu'ils se trouvent dans une situation qui peut les provoquer - forêts pour le pollen ou déserts pour le sable, par exemple.

80 Les Darcsen constituent un groupe ethnique aisément identifiable par ses traits reconnaissables, victimes d'un fort racisme dans le monde fictionnel de Valkyria Chronicles.
} 
Si certains éléments tendent vers une relative cohérence fictionnelle, il n'en demeure pas moins que Valkyria Chronicles sépare de façon très distincte son expérience narrative de son expérience de jeu. Alors que WoW et Fallout 3 font vivre les deux en même temps, ici on vit soit l'une, soit l'autre. On peut en fait diviser Valkyria Chronicles en trois phases distinctes - le récit, la planification (étape précédant le combat où sont expliqués les objectifs de la mission, et où l'on doit choisir quels membres de l'équipe il convient de déployer) et les missions, qui constituent l'essentiel du jeu. Les actions du joueur dans les phases «planification » et « mission », auxquelles il est amené à contribuer, n'ont aucun impact sur la phase «récit». À vrai dire, seuls la réussite ou l'échec des missions influencent le déroulement du récit : réussir implique la poursuite du jeu, échouer y met fin. ${ }^{81}$ Ainsi, soit on joue, soit on assiste au récit, présenté comme un film d'animation.

Qu'en est-il de la cohérence référentielle? Il ne s'agit pas d'un point fort du jeu, et la manœuvre semble, à tous égards, intentionnelle. Très fortement inspiré des jeux de stratégie, et non seulement électroniques, Valkyria Chronicles est régi par un système de règles qui s'apparente plus aux échecs qu'aux jeux de rôle traditionnels. Les missions sont pratiquement toutes une représentation d'un conflit armé, mais avec un fort degré d'abstraction. Chaque camp joue à son tour, disposant d'un nombre d'actions influencé par la présence d'unités de commandement (personnages spéciaux) sur le champ de bataille. Au cours d'un même tour de jeu, il est possible d'accumuler ces actions, ou

\footnotetext{
$81 C^{\prime}$ est ce que nous appellerions une structure d'actualisation, par référence à la théorie de l'enactment. Pour en savoir plus à ce propos, voir Anders Sundnes Lovlie - End of story? Quest, narrative and enactntent in computer games, présenté à la conférence <http://www.waikato.ac.nz/film/2005papers/319B/docs/Lovlie.pdf> (14 septembre 2011)
} 
encore de toutes les utiliser sur le même personnage. Certains personnages disposent de grenades, qu'ils récupèrent au début de chaque tour, malgré l'absence notable de corps de ravitaillement. Il s'agit là de deux exemples marquants d'une volonté de donner au jeu une forte dimension stratégique, la cohérence référentielle devenant dès lors un souci de (lointain) second ordre. Cette incohérence et la capacité de contrôler directement chacun des personnages empêchent tout effet de présence, particularité encore plus notable lorsqu'on remarque l'esthétique très peu photoréaliste du jeu :

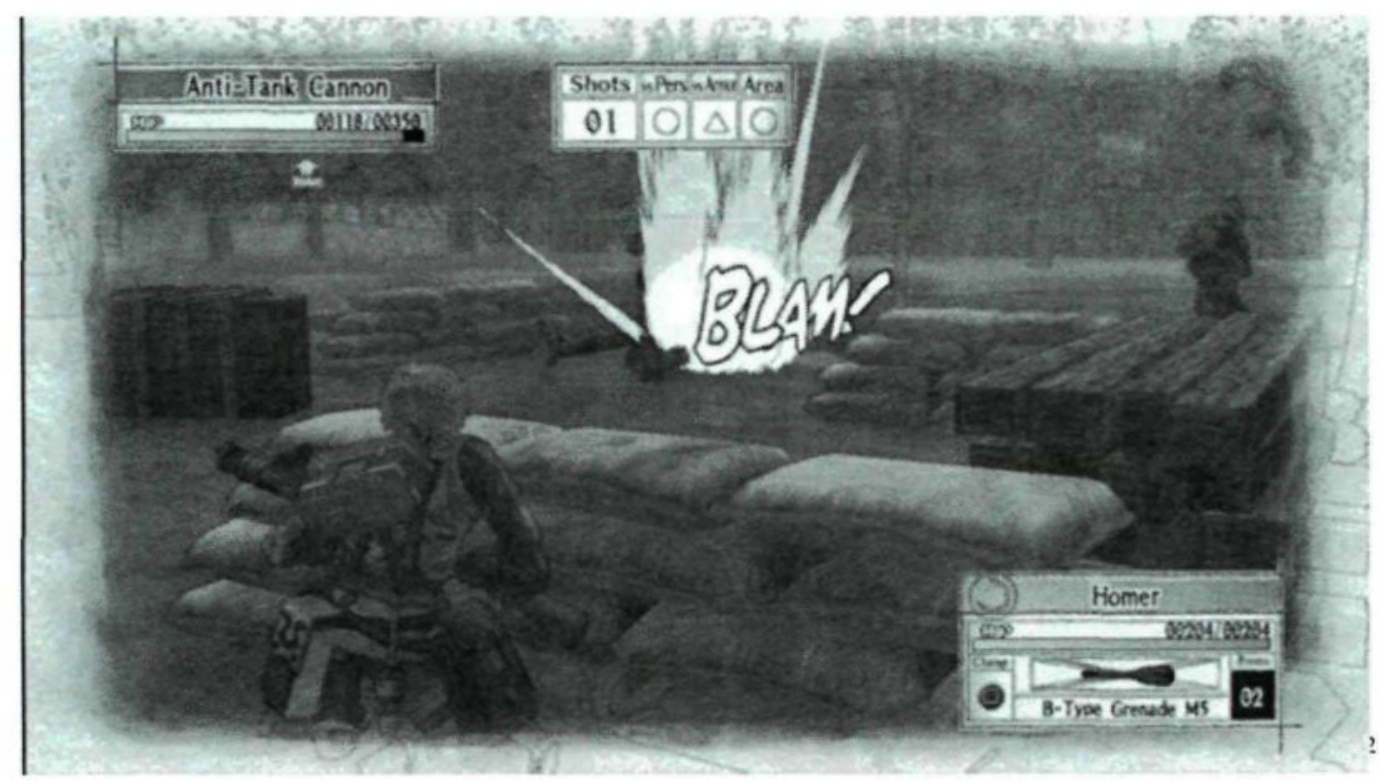

L'effet recherché est plutôt celui d'une immersion non diégétique, qui prend les règles de jeu comme un système fermé, consistant, permettant au joueur d'avoir un impact sur son environnement (le camp adverse, principalement). On peut même voir là un système qui répond fortement aux attentes de certains joueurs : ceux qui sont familiers avec les jeux de stratégie traditionnels et acceptent la logique derrière les mécanismes de jeu.

${ }^{82}$ Sega - Valkyria Chronicles. (The Kloden Wildwood - Battle of Kloden Forest) 
Le récit, lui, fonctionne à la façon d'une série d'animation. Il ne laisse aucune place aux décisions du joueur, et lui est présenté, d'une certaine façon, comme une récompense des efforts fournis jusqu'alors. Valkyria Chronicles invoque même consciemment l'imaginaire du livre d'histoire, se présentant comme un récit du passé, écrit à l'avance, comme on peut voir sur cette image :

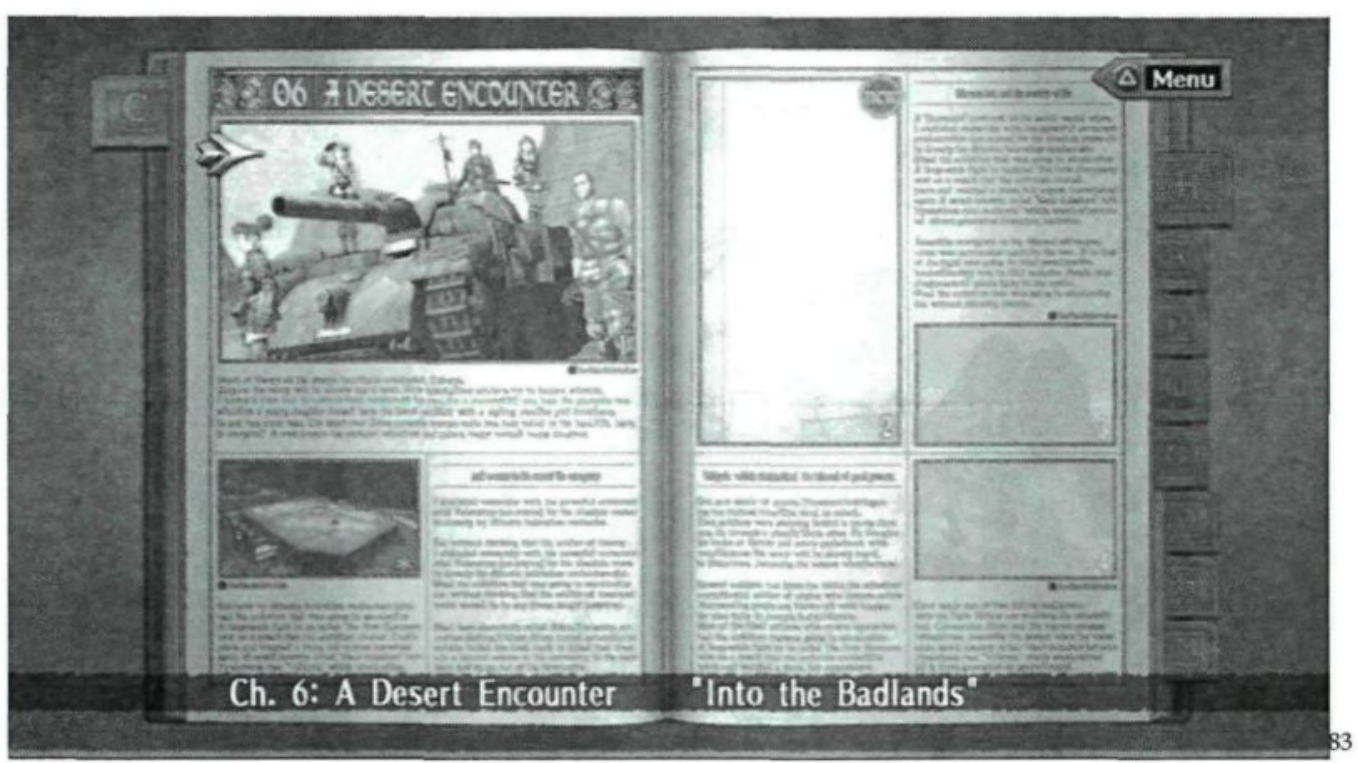

Le joueur ne fait que suivre les étapes du déroulement, et ses choix se limitent à rejouer un ancien passage, ou à poursuivre le récit.

Valkyria Chronicles offre donc une approche de la fictionnalité qui semble très traditionnelle - au sens où elle respecte les conventions de formes de récit qui datent d'avant les systèmes informatiques. Le jeu offre une petite latitude au joueur dans la détermination de quelques propriétés ponctuelles (forces et faiblesses de certains personnages) mais maintient un contrôle serré sur celles qui déterminent son expérience

${ }^{83}$ Sega - Valkyria Chronicles. (A Desert Encounter) 
narrative. Il s'agit du jeu qui cadre certainement le mieux avec l'idéal (vieilli) de Juul84 : ségrégation complète des deux expériences, ce qui permet au jeu de s'épanouir selon ses propres critères, plutôt que d'avoir à composer avec les impératifs de sa fictionnalité, ou même, dans ce cas, d'un rapport au réel.

\section{Conclusion}

Pour terminer cette analyse, nous aimerions rappeler que les trois jeux au corpus, bien que présentant des approches divergentes de notre sujet, demeurent plutôt similaires. Il s'agit de trois jeux de rôle, assez récents, et s'ils couvrent une gamme plutôt étendue du genre, ils demeurent un petit échantillon des jeux électroniques dans leur ensemble.

On a vu qu'il existait dans ce médium une tension entre plusieurs systèmes formels : les règles, gérant l'expérience de jeu; la fictionnalité, gérant l'expérience narrative; les lois de la physique et de la logique, gérant l'expérience du réel. Ces différentes instances peuvent, chacune à leur façon, créer un effet d'immersion (diégétique ou non diégétique) ou de présence. On a aussi établi qu'il était possible d’intégrer certains aspects, voire l'intégralité, de ces différents systèmes les uns aux autres - phénomènes baptisés cohérence fictionnelle et cohérence référentielle. L'analyse a montré qu'il n'est pas nécessaire d'élever en idéal l'intégration des systèmes pour créer l'effet d'immersion, et que certains jeux, au contraire, sont mieux servis par leur ségrégation.

${ }^{84}$ Dans Jesper Juul - A Clash Between Gante and Narrative. 
Valkyria Chronicles est ainsi un exemple de ségrégation des expériences. Très traditionnel, il traite le jeu et la fiction comme deux phénomènes dissociés, et alterne ainsi entre phases de jeu et phases de récit. World of Warcraft : Wrath of the Lich King amalgame les deux, mais est prêt à sacrifier un fort degré de cohérence pour permettre à son univers fictionnel et à son univers mécanique d'évoluer libres de toute contrainte. Fallout 3, lui, cherche à créer une expérience totale, intégrant autant que possible les trois systèmes formels. Nous avons pu voir, cependant, que la recherche de l'effet de présence y était reléguée au second plan, le jeu lui préférant une simplification de l'expérience.

Dans les trois cas, il s'agit d'approches de la fictionnalité spécifiques aux jeux électroniques. Certes, le récit de Valkyria Chronicles est indépendant de sa nature vidéoludique - il a même été adapté pour la télévision -, mais il demeure influencé dans une petite mesure par ses mécanismes de jeu. WoW nous montre, avec ses dix millions d'abonnés, que l'immersion diégétique peut être mise de côté sans nuire à la qualité (ou au succès) du jeu. Fallout 3 est la preuve qu'il est possible de laisser au joueur une énorme latitude dans la détermination des différentes propriétés fictionnelles et de créer un récit efficace, qui soit « à propos des choix du personnage »85 tout en développant un jeu célébré par la critique.

85 Josh Sawyer - «I thought I could organize freedom. How Scandinavian of me » (Nous traduisons) 


\section{CHAPITRE II}

\section{STRUCTURE DU RÉCIT, SÉQUENCES D'ACTIONS}

Nous avons établi précédemment un rapprochement entre les systèmes régissant la fiction et le jeu au sein des divertissements vidéoludiques, et ce faisant, posé que ces deux aspects sont, à importance variable, constitutifs du média. Prenant pour acquise la présence de fiction, nous avançons également que l'expérience de cette fiction est elle aussi essentielle au jeu électronique. Les règles fictionnelles (la fiction) mettent en place les conditions d'émergence de différentes situations représentées, qui peuvent être mises en intrigue : ce qui constitue, au final, un récit plus ou moins complexe. Notre objectif, ici, sera de comprendre le processus de mise en intrigue des différentes situations fictionnelles, et leur interaction avec les situations ludiques générées par les règles de jeu.

De prime abord, il nous semble nécessaire de définir lesdites situations. Marie-Laure Ryan, par exemple, nomme représentations narratives ce que nous avons identifié comme des situations, et les définit ainsi :

[L]es représentations narratives doivent être unifiées thématiquement et cohérentes logiquement. Leurs éléments ne peuvent être librement permutés, puisque qu'ils sont maintenus ensemble en une séquence par des relations de cause à effet, et parce que l'ordre temporel est significatif. Les propositions d'une représentation narrative doivent $s^{\prime}$ articuler autour d'un ensemble commun de référents. ${ }^{86}$

\footnotetext{
${ }^{86}$ Marie-Laure Ryan - «Beyond Myth and Metaphor : The Case of Narrative in Digital Media », Game Studies, Vol. 1, No. 1 (Juillet 2001). <http://www.gamestudies.org/0101/ ryan/> (11 septembre 2011)
} 
Une représentation narrative est donc un tout unifié, composée d'éléments organisés entre eux de façon séquentielle. La séquentialité, récurrente dans la littérature sur le sujet, est notamment présente dans la pensée de Jannidis, qui parle de «séquence d'événements organisés de façon chronologique et minimalement liés ».87 Si l'une parle d'éléments et l'autre d'événements, nous préférons aux deux le terme d'action, dans le sens que lui donne Gervais dans son ouvrage Récits et actions. ${ }^{88}$ Cela nous permet de cerner un peu mieux ce que nous voulions dire par situations représentées, mises en intrigue : il s'agit d'ensembles d'actions, unifiés et séquentiels, que Gervais qualifie de situation narrative une « entité [...] qui met en jeu, pour un cadre donné, un agent et son action. ${ }^{89}$

Avant d'explorer l'organisation des situations entre elles, commençons par développer l'organisation interne de chacune, suivant les termes de Gervais. Ce dernier définit le concept d'action à l'aide de ce qu'il nomme un schème interactif, un «modèle de la configuration générale de la pré-compréhension pratique de l'action ${ }^{90}$ qui tient un rôle double : «il sert [...] de régie des processus de compréhension [et] joue aussi le rôle de paramètre de la représentation discursive de l'action. »91 Si la représentation n'est pas que discursive dans le jeu électronique (ce qui au demeurant $n^{\prime}$ est pas problématique ${ }^{92}$, ce modèle demeure néanmoins un point de départ efficace.

\footnotetext{
87 Fotis Jannidis - « Event-Sequences, Plots and Narration in Computer Games ».

88 Bertrand Gervais - Récits et actions. Pour une théorie de la lecture.

89 Ibid., p.113.

90 Ibid., p.80.

91 Ibid., p.82.

92 D'autres ont déjà mieux défendu que nous l'aplanissement de la distinction entre représentations discursives et visuelles. Nous pensons notamment à Samuel Archibald, dans Le texte et la teclinique: la lecture à l'ère des nouveaux niédias, p.158-159.
} 


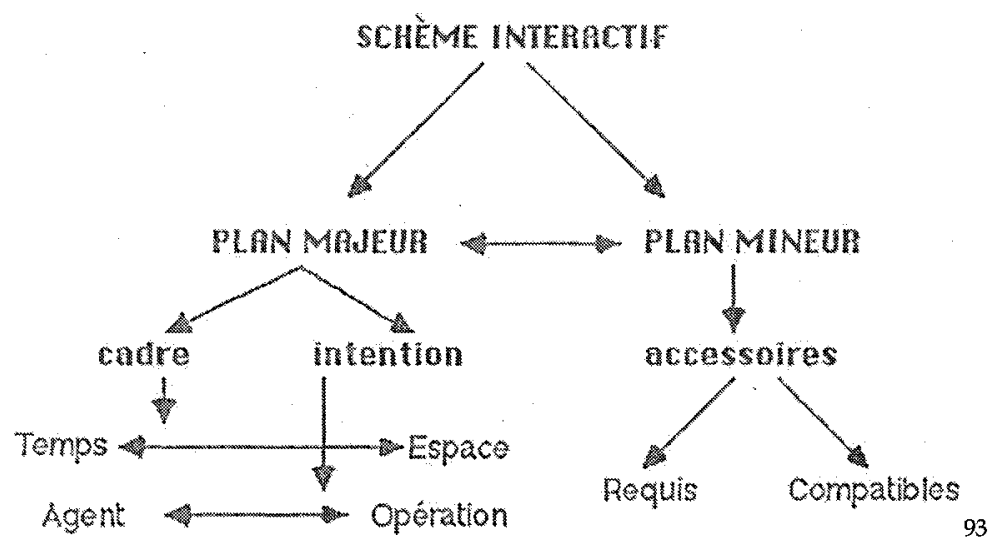

Une action, à entendre ici comme une tentative (avérée ou non) de transformation de l'état du monde par un agent anthropomorphe ${ }^{94}$, est donc composée de deux plans, mais ce sont surtout leurs éléments constitutifs qui nous intéressent : cadre, intention et accessoires.

Les accessoires, d'abord, qu'ils soient requis ou compatibles, constituent ce que Gervais appelle un plan mineur, et sont nécessaires considérant que «certaines actions ne se comprennent [...] que dans leur relation aux accessoires qu' elles utilisent. „95 $\mathrm{Il} \mathrm{s}$ 'agit donc d'éléments pouvant (voire devant) être représentés ou inférés dans le cadre d'une action. Ils demeurent d'une importance secondaire, mais sont une composante nécessaire de la conceptualisation de l'action.

Le cadre, lui, « fournit l'environnement nécessaire à la représentation. »96 Gervais identifie deux éléments principaux du cadre: le temps et le lieu. Il demeure impliqué dans la

\footnotetext{
${ }_{93}$ Ibid., p.81.

94 Il convient de noter que la notion d'anthropomorphie n'est pas aussi discriminante qu'elle peut l'apparaître, comme on peut le constater dans la défense qui en est faite dans Samuel Archibald - Le texte et la technique : la lecture à l'ère des nouveaux médias, p.159-160.

95 Ibid., p.110.

96 Ibid., p.81.
} 
construction et l'interprétation des actions, qu'il soit géographique (déserts et océans suggèrent des idées bien différentes face à l'action « se laver », par exemple) ou temporel (une œuvre de science-fiction cyberpunk permet nombre d'actions qui seraient improbables dans un roman historique situé à l'époque médiévale). Il ne s'agit pas seulement d'un contexte dans lequel s'inscrit l'action, déterminant principalement pour sa représentation et sa compréhension : il fournit un ensemble de références qui rendent plus ou moins vraisemblables diverses actions. Il est ainsi intimement lié à l'éventail des actions possibles. Nous nous y attarderons ici surtout comme condition préalable à l'action.

L'intention, elle, est définie par Gervais comme « la composante essentielle de 1'action. »97 Elle fait appel à quatre notions qui composent le « portrait intentionnel $»^{98}:$ le moyen, le but, le motif et le mobile. Le couple moyen/but donne lieu à une opération (on utilise un moyen pour obtenir un but) alors que le couple motif/mobile « se rattache directement à l'agent et permet de déterminer ses motivations, les raisons qui le poussent à agir. »99 Gervais distingue motif et mobile ainsi :

Les mobiles sont de l'ordre du vécu, ils recouvrent les raisons, les déterminations psychologiques ou socio-culturelles qui ont incité à agir; aussi sont-ils rétrospectifs. Les motifs, eux, sont d'ordre intellectuel, ils sont les raisons pour agir, la source de ces actions entreprises afin d'atteindre les buts visés; ils sont prospectifs. ${ }^{100}$

\footnotetext{
97 Ibid., p.103.

98 Ibid., p.103.

99 Ibid., p.96.

100 Ibid., p.96-97.
} 
L'intention devient ainsi, pour Gervais, la relation entre le couple motif/mobile et le couple moyen/but: il s'agit d'un ensemble de conditions et de raisons qui mènent à l'action, et aux modalités d'accomplissement de cette dernière. Elle est le rapport entre un agent et son opération.

$\mathrm{Au}$ final, en ce qui concerne la conceptualisation de l'action, Gervais propose de considérer l'expression discursive d'un schème interactif (dont nous venons d'explorer les composantes) comme une situation narrative. 101 Il s'oppose ainsi à un modèle du récit qui présente la situation narrative comme un état statique, et l'action comme la transformation de cet état en un autre. Pour lui, «la situation recouvre l'action et elles sont dans une relation de subordination plutôt que de complémentarité. »102 $C^{\prime}$ est donc à travers l'action que s'exprime le récit, qui est pensé « comme un ensemble de situations se succédant au rythme des actions accomplies. „103 Pour le dire brièvement, l'action est l'unité de base de la situation narrative, et c'est l'enchaînement d'une situation à l'autre qui donne lieu au récit. Cette pensée nous ramène à l'objectif de ce chapitre, à savoir les modalités selon lesquelles les situations narratives s'enchaînent dans le jeu électronique. C'est pourquoi nous désirons poursuivre avec les théories de Gervais, qui offre une vision compréhensive des possibilités d'enchaînement des situations narratives, telles qu'il les conceptualise.

\footnotetext{
${ }^{101}$ Ibid., p.113-126.

102 Ibid., p.124.

${ }_{103}$ Ibid., p.124.
} 
Pour qu'il y ait enchaînement, il doit y avoir au minimum deux situations narratives distinctes, qui se distinguent pour Gervais par « la variation d'un des quatre éléments du plan majeur du schème interactif »104: le temps, le lieu, l'agent et son portrait intentionnel.105 Un récit est ainsi une suite de situations distinctes, la première devenant la « situation narrative inaugurante, et la dernière la situation narrative finale »106 avec entre les deux « un ensemble de situations intermédiaires, enchaînées les unes aux autres. »107 Cette vision du récit comme une consécution de différentes situations narratives vient faire écho au sentiment de Ryan concernant les représentations narratives : elles sont nécessairement séquentielles, et leur ordre est signifiant. Ryan discute également de la nécessité d'un «ensemble commun de référents »108: c'est la présence de cet ensemble commun qui permet de considérer différentes situations comme appartenant à la même séquence. Ainsi, nous pouvons dire qu'un récit présente au moins une action, qui se subdivise en plusieurs situations narratives présentant un ensemble commun de référents, et qui s'organisent de façon séquentielle.

Si les situations narratives s'organisent effectivement de façon séquentielle afin de former un récit, elles peuvent néanmoins s'enchaîner de différentes façons, et tenir un rôle différent dans ledit récit. Gervais propose deux statuts possibles : celui de situations

\footnotetext{
104 Ibid., p.194.

105 Ici, Gervais emploie plutôt le terme de plan-acte, qui est une élaboration du concept de portrait intentionnel. Nous retenons pour nos besoins l'idée de portrait intentionnel, qui englobe les mêmes éléments, car nous ne jugeons pas nécessaire dans le cadre de notre réflexion d'entrer dans le détail du plan-acte tel qu'exploré par Gervais.

106 Ibid., p.194.

107 Ibid.

108 Marie-Laure Ryan - «Beyond Myth and Metaphor : The Case of Narrative in Digital Media ».
} 
narratives directrices et celui de situations narratives subordonnées. ${ }^{109}$ Les deux sont définies comme suit :

Les situations directrices sont celles qui, en se succédant et se côtoyant, forment la ligne principale du récit. Elles peuvent s'enchaîner dans une progression linéaire simple $[. .$.$] ou se déployer en de multiples plans. [...]$

Les situations subordonnées sont celles qui, plutôt que de faire progresser le déroulement de l'action, permettent au contraire d'en expliquer les particularités,

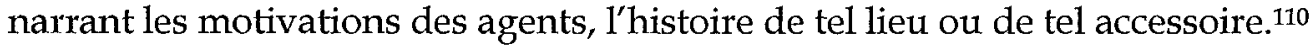

Concernant les possibilités d'enchaînement, Gervais propose de nombreuses modalités. Il parle notamment de consécution simple, d'enchaînement par les moyens, d'enchaînement par les buts, d'enchaînement par le cadre ainsi que de subordination.111 Dans tous les cas, il revient sur les quatre éléments du schème interactif (temps, lieu, agent, portrait intentionnel), et l'enchaînement ne peut s'opérer qu'avec un référent commun, un élément qui sert en quelque sorte de repère - permanence de l'agent, ou du but, par exemple. La subordination est un cas particulier, concernant les situations subordonnées qui cherchent à détailler un des éléments de l'action. Gervais parle aussi de situations parallèles, arguant ainsi qu'un récit peut, de fait, alterner entre différentes séquences qui forment chacune un récit propre.

Résumons: un récit est la consécution de situations narratives, passant d'une situation inaugurante à une situation finale à travers des situations intermédiaires. Deux situations contiguës présupposent au moins un référent commun, un élément stable, et s'enchaînent

\footnotetext{
109 Voir dans Bertrand Gervais - Récits et actions. Pour une théorie de la lecture, p.195-222.

110 Ibid., p.195.

111 Voir Ibid., p.195-222.
} 
suivant la variation d'au moins un des quatre éléments du plan majeur du schème interactif : temps, lieu, agent, portrait intentionnel. $\grave{A}$ cette trame principale peuvent se greffer des situations subordonnées, qui n'affectent pas le déroulement du récit, mais éclairent un des éléments de $1^{\prime}$ action. Un récit peut même être constitué de plusieurs séquences d'actions en alternance, avec chacune leurs modes d'enchaînement.

Le jeu électronique présente cependant plusieurs caractéristiques qui viennent se greffer à ce modèle. Nous avons déjà montré, au premier chapitre, qu'au cœur de l'expérience vidéoludique se trouvent deux systèmes de règles qui se répondent et s'influencent : un système de règles ludiques, qui construit le jeu, et un système de règles fictionnelles, qui construit le récit. Il va de soi qu'une conception de la structure du récit vidéoludique ne peut se faire qu'au regard de cette interaction entre jeu et fiction. Ainsi, si nous voulons étudier l'action et ses enchaînements possibles, nous devons notamment prendre en considération le fait que chaque action accomplie par un agent contrôlé par le joueur peut $s^{\prime}$ interpréter dans le cadre des règles fictionnelles comme dans le cadre des règles ludiques - et c'est là qu'il nous faut élargir l'approche de Gervais.

La notion d'agent, particulièrement, est problématique au sein d'une ouvre vidéoludique, lorsqu'on parle d'un agent fictionnel contrôlé par le joueur : chaque action accomplie par le premier est nécessairement liée à une action accomplie par le second (les deux sont donc agents) mais elles n'obéissent pas nécessairement au même schème interactif. Cette façon dédoublée de considérer l'action vidéoludique fait écho à la nature double du jeu 
électronique présentée au premier chapitre - fiction et jeu deviennent ici personnage et joueur, et chaque action dédoublée accomplie peut s'interpréter suivant le portrait intentionnel de l'un ou de l'autre. Le héros de Baldur's Gate"112, par exemple, règle de nombreux conflits pour différentes raisons - grandeur d'âme, soif de pouvoir ou de vengeance, etc. - et ses agissements s'inscrivent évidemment dans l'univers fictionnel. Le joueur qui le commande, par contre, peut tout à fait choisir les conflits à régler selon leur récompense en points d'expérience, une mesure qui n'a de sens qu'au plan des règles du jeu. Ainsi, l'action, dans ce cas-ci, peut être comprise comme résultant des intentions de l'agent fictionnel (le personnage) ou de celles de l'agent ludique (le joueur) - voire des deux à la fois.

Sur le plan de l'enchaînement des situations narratives, cela veut dire que la présence d'un agent-joueur et $d^{\prime} u n$ portrait intentionnel du joueur vient élargir les possibilités de référents communs justifiant l'enchaînement de différentes situations narratives. Une séquence d'actions, par exemple, pourrait théoriquement n'avoir en commun que l'agentjoueur, et demeurer une représentation unifiée, un récit en quelque sorte - si on se réfère au système de règles ludiques. Ou encore, une situation subordonnée pourrait n'offrir qu'un élargissement des capacités du joueur, ou ne développer que les capacités ludiques du personnage : les errances de personnages en quête de points d'expérience, par exemple, sont familières aux joueurs de Dragon Quest, mais ne s'intègrent au récit que si on considère leur dimension ludique. C'est donc dire qu'en acceptant la nature double du jeu

112 BioWare - Baldur's Gate, Interplay, 1998. 
électronique, il nous est possible de repérer la séquentialité à la fois face à la fiction et face au jeu, et d'ainsi comprendre le récit vidéoludique comme à la fois un récit et une expérience de jeu, qui s'imprègnent l'un de l'autre.

Il faut cependant rappeler que l'expérience de jeu et le récit ont été longtemps pensés comme incompatibles. C'était par exemple cette prétendue différence essentielle entre les deux qui poussait Juul (dans ses premiers travaux) à refuser toute analyse portant sur la narrativité du jeu électronique. ${ }^{113}$ L'argument essentiel était que le récit, par nature, est linéaire et séquentiel (ce qui fait écho à ce dont nous avons discuté plus tôt) tandis que le jeu est intrinsèquement libre, et qu'un jeu linéaire n'est, somme toute, qu'un récit déguisé. Le refus de la séquentialité pour créer une bonne expérience de jeu se retrouve également chez les créateurs eux-mêmes, comme Sawyer qui suggère qu'un bon jeu doit « permettre au joueur d'accomplir ce qu'il ou elle désire au sein d'une histoire ouverte, plus grande. »114 Autrement dit, si les deux systèmes peuvent également supporter la linéarité, le récit la fait sienne tandis que le jeu la rejette. Les jeux électroniques à caractère fictionnel, cependant, doivent nécessairement faire cohabiter les deux expériences. Notre analyse, notamment, nous amènera à repérer de quelle façon chaque œuvre compose avec les exigences et tendances du récit et du jeu, et de quelle façon chacune met de l'avant une structure basée sur l'un ou l'autre.

113 Voir à cet effet Jesper Juul - A Clash between Game and Narrative.

114 Josh Sawyer - «I thought I could organize freedom. How Scandinavian of me » (Nous traduisons) 
Nous avons mentionné précédemment que plusieurs caractéristiques propres au vidéoludique poussent à compléter l'approche de Gervais; nous désirons maintenant en examiner une dernière. Certains jeux refusent la notion même d'expérience balisée, avec un début, un développement, et une fin - ce sont ces jeux que Douglas appelle les « livres sans fin. »115 Cette position force à penser le récit autrement qu'en termes d'une trame principale, qui suppose une situation inaugurante et une situation finale.

S'il peut être difficile d'identifier une trame principale fictionnelle unique dans certains jeux (nous pensons en particulier aux jeux multijoueurs en ligne), il n'en demeure pas moins que l'expérience de jeu, elle, est nécessairement balisée, avec un début et une fin discernables. La trame principale, à ce moment, est liée à la permanence de l'agent-joueur, pour qui chaque action fait partie du même jeu. Ces actions, ou situations narratives, ne s'inscrivent cependant pas, comme nous venons de le mentionner, à l'intérieur d'un récit unique - ce qui ne veut pas dire qu'elles ne s'inscrivent pas à l'intérieur d'un récit. En effet, bien que certains jeux ne présentent pas une trame ou un récit fictionnel uniques, ils peuvent néanmoins regrouper leurs différentes situations narratives, et leur permettre de s'enchaîner en séquences narratives - autrement dit: en courts récits. Par exemple, la seconde moitié du jeu de rôles japonais Final Fantasy III (version américaine) ${ }^{116}$ est formée de séquences optionnelles de jeu, qui constituent chacune en soi un court récit (retrouver un ami perdu, sauver un village) mais qui ne sont intrinsèquement ni liées l'une à l'autre,

\footnotetext{
115 Jane Yellowlees Douglas - The End of Books - Or Books without End? Reading Interactive Narratives, The University of Michigan Press, Ann Arbor, 2001. 116 SquareSoft - Final Fantasy III, SquareSoft, 1994.
} 
ni tenues par un unique fil conducteur. Une approche du récit vidéoludique qui considère les différentes actions comme faisant partie non pas nécessairement d'un tout fictionnel, mais plutôt de sous-ensembles liés par leur appartenance à la même expérience de jeu, a déjà été proposée par Jannidis; nous allons maintenant nous y attarder.

Se concentrant principalement sur les jeux en ligne, Jannidis identifie la quête comme élément structurant essentiel au jeu. Il s'agit d'une association tout à fait sensée : on a depuis longtemps considéré la résolution de problèmes (présentée ici sous forme de quête) comme modèle de base du jeu. ${ }^{117}$ Pour nous, cependant, il ne s'agit que d'une organisation possible d'actions, un ensemble qui peut servir de modèle de base au récit vidéoludique non unifié. Il faut spécifier que Jannidis lui-même ne limite pas son analyse à la quête, qui n'est qu'une possibilité. Les quêtes demeurent des « séquences relativement indépendantes d'événements et cela signifie qu'il n'est pas important pour le jeu qu'on suive ou non une quête »118, du moins dans les jeux qu'il étudie - nous verrons dans notre analyse que certaines cuvres développent des relations autres avec la quête.

Chaque quête, inévitablement, est en soi un récit, une séquence qui demande au joueur un certain enchaînement d'actions, générant ainsi au sein de la fiction une série de situations narratives, avec une structure interne qui rappelle le modèle de Gervais. Nous proposons de nommer ces situations narratives des « rencontres », qui peuvent donc être organisées

\footnotetext{
117 Notamment, Johan Huizinga - Homo Ludens: Essai sur la fonction sociale du jeu et Roger Caillois - Les Jeux et les hommes : le masque et le vertige abordent le sujet.

118 Fotis Jannidis - «Event-Sequences, Plots and Narration in Computer Games ». (Nous traduisons)
} 
sous forme de quête, comme des situations soit directrices (elles font avancer la quête), soit subordonnées (elles ne font que développer le personnage ou un autre élément de l'action). On peut résumer une rencontre à une interaction entre les éléments contrôlés par le joueur (personnage, forces armées) et ceux dirigés par l'intelligence artificielle (ennemis, obstacles, alliés non-joueurs) ou encore passifs (une montagne à grimper). Le déroulement d'une rencontre peut s'exprimer ainsi: une série d'actions entreprises par le joueur/personnage qui se confronte à une série d'actions programmées par le système, créant ainsi une situation dramatique, définie par Gervais comme « la rencontre conflictuelle d'au moins deux forces ${ }^{119}$. La situation de combat est un exemple courant de rencontre : une quête demandant l'élimination de la menace de bandits, par exemple, se résout bien souvent par une série de rencontres (conflits armés) entre le(s) protagoniste(s) et lesdits bandits.

L'approche de Jannidis n'est, au final, qu'une autre façon de nommer les mêmes éléments identifiés par Gervais. Elle a cependant l'intérêt de refuser la notion d'un tout englobant, préférant percevoir les différents sous-récits présents dans le jeu électronique comme des séquences relativement indépendantes. Mieux encore, cette approche relève l'interaction particulière entre expérience de jeu et récit, en posant que la quête, en tant que récit, «peu[t] offrir des possibilités de signification au joueur, mais il [le joueur] peut et va également accepter plusieurs autres possibilités. »120 Jannidis suppose ici que le sens fictionnel n'est pas la seule façon d'explorer l'expérience de jeu, qui ne se limite donc pas

\footnotetext{
119 Bertrand Gervais - Récits et actions. Pour une théorie de la lecture, p.115.

120 Fotis Jannidis - «Event-Sequences, Plots and Narration in Computer Games ». (Nous traduisons)
} 
au récit - le joueur peut ainsi voir sens, structure et enchaînement sur d'autres plans : celui des règles de jeu, par exemple.

Bref, nous pourrons utiliser quête au sens de récit, ou encore rencontre au sens d'action. L'essentiel à retenir est que, pour Jannidis, le récit vidéoludique se décline non pas comme un ensemble, mais comme une grappe de sous-ensembles. Ces derniers peuvent être liés entre eux pour former la trame principale d'un grand récit, mais ils peuvent tout autant être parfaitement indépendants, n'étant liés que par leur appartenance à la même expérience de jeu.

Ce sont là tous les outils dont nous aurons besoin pour analyser les modalités de la structure du récit vidéoludique. Pour chaque œuvre, nous essaierons de voir comment s'articulent les différentes actions possibles, et de quelles façons elles peuvent s'enchaîner, qu'on les considère comme des situations narratives ou comme des rencontres. Une attention particulière sera accordée à la trame principale de chaque récit (ou à son absence), pour voir de quelle(s) façon(s) il est possible de naviguer de la situation inaugurante à la situation finale, à travers quelles situations intermédiaires. Nous chercherons aussi à voir comment se subordonnent les différentes actions qui ne s'inscrivent comme situation directrice dans aucune trame (si elles existent). Surtout, nous interrogerons la position de chaque ouvre face à la linéarité, associée au récit, et à la libre exploration, associée au jeu. Nous chercherons donc à voir comment chacune jongle avec les possibilités offertes par le support informatique. 


\section{Fallout 3}

On décrit souvent Fallout 3 et autres jeux similaires comme des « bacs à sable » ouverts, où le joueur a pour mission d'écrire lui-même son histoire. Du point de vue de la structure du récit, toutefois, il s'agit d'une exagération. Le système présente un objectif final, une progression claire et même (parfois) linéaire, et génère sans contribution du joueur les nombreuses quêtes, officielles ou non, qui motivent ses actions et celles de son avatar.

Le grand objectif du jeu, sa quête centrale, n'est cependant pas annoncé ouvertement dès le départ. Le jeu n'informe plutôt le joueur que d'une étape de son récit à la fois, et n'impose pas de suivre la séquence proposée - ce n'est qu'avec le recul final qu'il devient aisé de cerner la trame principale qui dirige le récit. Par exemple, une fois la première section $\mathrm{du}$ jeu traversée (la jeunesse du personnage, qui fait office de moment d'apprentissage), le joueur est catapulté sur les traces de son père, disparu un peu plus tôt. Bien qu'elle soit présentée comme l'objectif principal de départ, cette quête peut être ignorée : le monde peut être exploré librement, sans égard pour la quête principale, ni aucune autre. Le personnage est néanmoins toujours amené à faire de nombreuses rencontres, pouvant se présenter comme des situations directrices de la quête principale (un marchand donne au personnage des informations sur son père) ou subordonnées (le personnage est attaqué par des chiens mutants). La recherche du père peut se faire en de nombreuses étapes, mais il peut tout autant être retrouvé par hasard, ce qui rend la plupart des quêtes, même la principale, optionnelles, comme dans la vision défendue par 
Jannidis. Une fois la situation inaugurante dépassée, l'enchaînement de différentes situations intermédiaires tombe donc entre les mains du joueur.

Il est au moins une quête, cependant, qui est inévitable : celle qui amène à la conclusion du jeu. Les autres, en grande majorité, peuvent être ignorées, et le jeu peut n'être perçu que comme une série de rencontres liées uniquement par le personnage qui les vit. Plus encore, il est possible pour le joueur de choisir les quêtes qu'il veut accomplir, l'ordre dans lequel elles le seront, et même (parfois) leurs modalités de résolution. Chaque quête déploie dans la fiction un schème interactif, avec un portrait intentionnel suggéré pour le personnage et, comme incitatif pour le joueur, contribue à l'avancement du personnage en lui conférant expérience et ressources diverses. Ainsi, le rôle du joueur est mis de l'avant dans l'organisation des quêtes entre elles, mais non dans leur conception, qui reste l'apanage du jeu. L'expérience complète de jeu est donc constituée d'une introduction et d'une conclusion fixes (situations inaugurante et finale), avec un développement (enchaînement de situations intermédiaires) qui relève des choix du joueur à propos des quêtes et rencontres permises par le jeu.

Nous considérons ce type de structure comme une structure arborescente dirigée. Elle est foncièrement arborescente (ses différents éléments peuvent être parcourus de nombreuses façons) mais elle comporte un élément plutôt dirigé : un point de départ, un point final, et quelques points d'ancrage entre les deux. L'enchaînement en arborescence dirigée est conçu de façon à ce que le joueur puisse parcourir librement (ou ignorer) les quêtes situées 
entre différents points d'ancrage, explorer à sa guise, et ainsi parvenir au prochain point de la façon qui lui convient le mieux. On peut donc choisir la façon dont progresse le récit. Il s'agit néanmoins d'une douce illusion : tous les chemins, au final, mènent à Rome, mais il existe des routes plus longues que d'autres pour y parvenir, et le choix est dicté par des contraintes autant fictionnelles (le personnage doit explorer pour trouver les traces de son père) que ludiques (chaque quête supplémentaire développe les attributs du personnage).

Les chemins n'étant donc pas tous égaux en termes ludiques et permettant à des personnages de niveau de puissance grandement différents de se situer au même point dans le récit, il est naturel que Fallout 3 adapte ses quêtes obligatoires pour convenir à des personnages de puissances très variables. C'est pourquoi la majorité des rencontres, qu'elles fassent ou non partie d'une quête, sont basées non seulement sur le cadre géographique, d'ordre fictionnel (on ne rencontre des fourmis géantes que près des nids de fourmis, par exemple) mais aussi sur le niveau d'expérience du personnage, qui sert de cadre ludique. De cette façon, toute action entreprise par le joueur crée nécessairement une certaine tension, puisque les agents non joueurs sont (presque) toujours d'une puissance comparable. Cela assure que, malgré l'éventuelle non-linéarité de la progression du récit, la progression ludique, elle, suit une courbe constante, avec une évolution graduelle des capacités du personnage et du joueur, qui doit résoudre des problèmes de difficulté croissante. 
Ce ne sont cependant pas toutes les quêtes et les rencontres qui participent à la trame principale de Fallout 3. Certaines y contribuent clairement (aller explorer l'ancien laboratoire de recherche du père) et d'autres plus subtilement (une mission effectuée pour la Brotherhood of Steel qui semble un pur acte mercenaire prépare en fait la quête finale en faisant connaître le personnage de cet organisme influent), mais plusieurs ne sont que décoratives, au moins pour la fiction. Il existe donc, en plus de l'arbre principal, des grappes d'actions possibles parsemées un peu partout dans l'univers de jeu, des séquences indépendantes, qui peuvent être perçues comme subordonnées, ne servant qu'à détailler un cadre, un opposant ou à développer les capacités du joueur et de son personnage.

La résolution des quêtes et des rencontres - donc les actions individuelles du personnage et du joueur - laisse beaucoup plus de place à la liberté du joueur. Ainsi, la première quête, dont l'objectif est de trouver de l'information sur le père, peut se dérouler de différentes façons: le personnage peut payer le barman local pour qu'il lui donne l'information, infiltrer son ordinateur pour y trouver un dossier qui lui apprend la même chose, séduire la petite amie du dit barman et lui faire cracher le morceau, ou même assassiner cette dernière et trouver une note dans ses effets personnels. Plus encore, il est possible d'échouer dans la quête, de poursuivre le récit à l'aveuglette et de parvenir quand même à retrouver le père, sans jamais avoir eu accès à cette information. La plus banale rencontre avec un marchand peut se dérouler de façon diplomatique, être négociée à la pointe de l'épée ou encore se solder par un vol à la tire. Fallout 3 pousse ainsi la structure en arborescence autant que possible : au sein même de chaque quête, plusieurs chemins 
sont possibles pour obtenir le résultat souhaité. Et l'idée n'est pas nouvelle : le premier jeu de la série Fallout peut être complété sans jamais combattre un seul ennemi, fait plutôt surprenant considérant que la seule progression ludique possible doit se faire par l'acquisition d'expérience de combat. C'est donc dire qu'étonnamment, alors que le jeu réduit autant que possible la linéarité inhérente à la narration fictionnelle, il permet également de rejeter entièrement la progression ludique. L'accent est donc mis sur la liberté du joueur.

Il ne faut pas croire, cependant, que toute action est permise. Comme toujours, les systèmes de règles ludiques et fictionnelles délimitent ce qui est possible, et ce qui ne l'est pas. Certaines résolutions ne sont tout simplement pas autorisées par le programme. D’autres sont fortement suggérées, sans être l'unique voie possible :

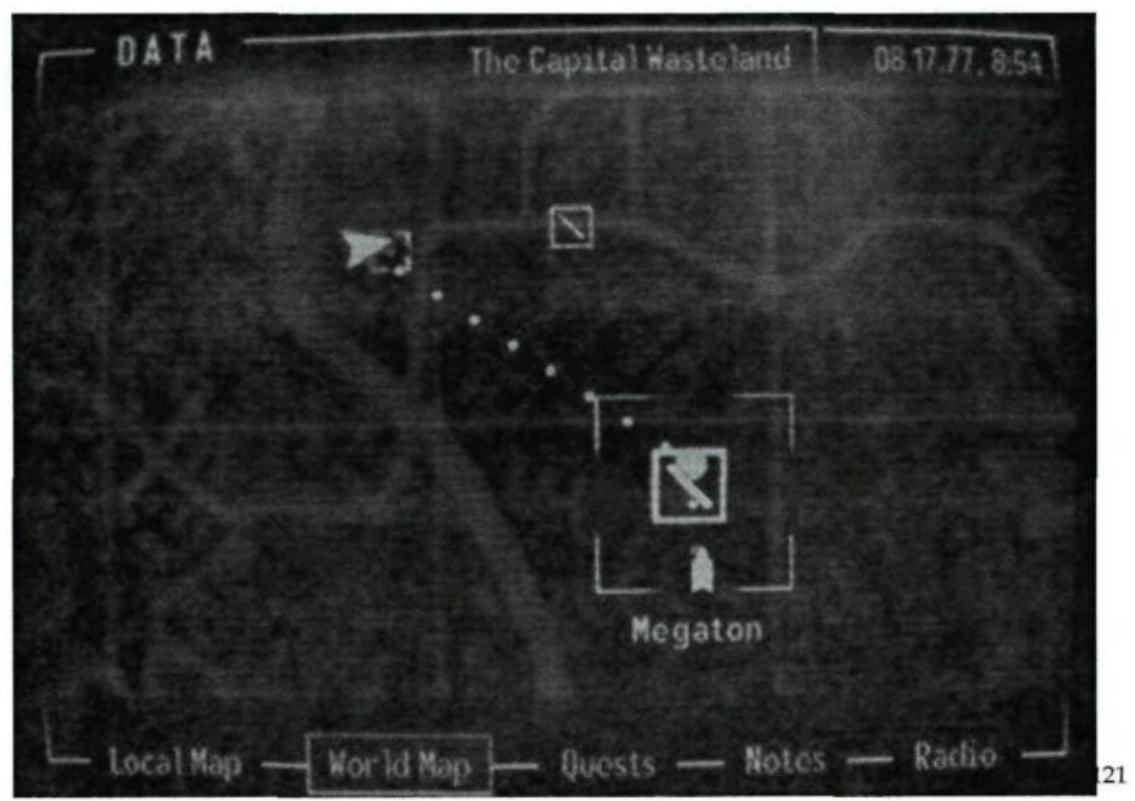

${ }^{121}$ Bethesda Game Studios - Fallout 3. (The Capital Wasteland - Following in His Footsteps) 
Il s'agit ici de la carte d'une zone extérieure, telle que présentée par l'interface de jeu. La flèche en haut à gauche représente la position du personnage, celle en bas à droite marque l'emplacement de l'objectif de la quête. La quête en question est celle dont nous avons parlé précédemment, liée à l'information sur le père disparu. Outre celui indiqué sur ce plan, il existe de nombreux autres endroits où on peut retrouver 1 'information - celui-ci est simplement, à ce moment, le plus proche. Ce mécanisme de suggestion ne réduit pas les possibilités offertes par le jeu - il faut plutôt le voir comme une aide précieuse pour le joueur qui ne désire pas avoir à deviner de quelle façon le récit peut progresser. L'action est suggérée sans être imposée.

En général, on peut dire que la structure narrative de Fallout 3, autant en ce qui concerne la trame principale que les ensembles plus petits d'actions, obéit à la logique de la structure en arborescence dirigée dont nous avons parlé. Il existe une quête principale, et un chemin le plus court pour naviguer d'une action à l'autre, mais il existe aussi de nombreuses autres possibilités de séquences. Les grandes lignes sont tracées, mais plusieurs façons de les parcourir existent; il appartient au joueur de décider quelle sera la sienne, et celle de son personnage. Ainsi, le jeu résout la tension entre linéarité et liberté d'exploration en demeurant linéaire si on le perçoit comme un grand ensemble, un récit unique, mais en remettant entre les mains du joueur les méthodes d'enchaînement, qui n'a pas à être linéaire ou chronologique. 


\section{World of Warcraft : Wrath of the Lich King}

L'architecture du récit de World of Warcraft, étant donné son statut de monde persistant, est fondamentalement éclatée. Le jeu n'a pas, à proprement parler, de finalité en cause pour nous permettre $d$ 'insérer ou non les diverses actions possibles dans une trame principale. Sans véritable objectif final, World of Warcraft est un de ces « livres sans fin » encensés par la narratologie vidéoludique ${ }^{122}: c^{\prime}$ est donc dire que nous nous trouvons face à une œuvre qui nous force à repenser une lecture traditionnelle en séquences narratives linéaires. Il est toutefois possible d'identifier une certaine progression dans le récit, mais, comme nous allons le voir, elle n'obéit que très peu aux modèles habituels.

Les différentes quêtes et actions à accomplir dans un monde persistant ne sont peut-être pas aisément liées entre elles d'un point de vue fictionnel, mais elles obéissent à une logique ludique. Il est apparent, par exemple, que World of Warcraft cherche à inscrire chaque élément de jeu au sein de la progression ludique du personnage mené par le joueur. Chaque ennemi vaincu ou chaque quête résolue, par exemple, vaut au joueur différentes ressources (expérience, richesses, armes, honneur) qui sont ensuite utilisées pour améliorer les différentes compétences du personnage. Même un banal détour chez le marchand permet d'échanger les ressources inutilisables en éléments de jeu qui permettent d'améliorer le personnage. Ainsi, nous proposons de considérer l'amélioration de ce dernier comme le fil conducteur qui lie les diverses actions proposées par le programme. $D^{\prime}$ un point de vue fictionnel, il ne s'agit pas toujours d'une position

122 L'expression, et la pensée, sont empruntées à Jane Yellowlees Douglas - The End of Books - Or Books without End? Reading Interactive Narratives. 
défendable ${ }^{123}$, mais, d'un point de vue ludique, cette perspective est incontestablement valide. Cela signifie que le schème interactif $\mathrm{du}$ personnage, qui contribue habituellement à comprendre l'enchaînement des actions au sein du jeu, est ici en majeure partie supplanté par celui du joueur.

L'amélioration des capacités du personnage se mesure de différentes façons. Son indicateur le plus aisément identifiable est certainement le niveau d'expérience, qui augmente à mesure qu'il accomplit diverses actions dans le jeu. Une fois le niveau maximal atteint, cependant, la progression semble s'arrêter ${ }^{124}$ - mais en réalité, elle se poursuit sur d'autres plans. World of Warcraft base la progression du joueur (après qu'il a atteint le niveau maximal) sur l'équipement (armes et armures), qui peut être obtenu par une panoplie de méthodes. Il est possible d'en acheter, d'en fabriquer soi-même, d'en trouver sur des ennemis difficiles à abattre, d'échanger des marques obtenues en combat organisé entre joueurs contre des objets plus puissants, et bien d'autres encore. L'objectif principal des actions accomplies au niveau maximal (et même avant, parfois) demeure toutefois l'obtention d'équipement permettant l'amélioration des performances au combat du personnage.

123 Si certaines quêtes, surtout en début de partie, se veulent explicitement un processus d'apprentissage et de progression, beaucoup sont constituées de requêtes envers un héros, qui (d'un point de vue fictionnel) n'a rien à y gagner. C'est là que la progression ludique prend toute la place, le gain d'expérience ou de réputation envers certains clans devenant la récompense motivant la quête.

124 Il faut aussi spécifier que le niveau maximal augmente à la sortie de chaque extension de jeu, repoussant ainsi les limites de ce type de progression. Toutefois, tant qu'il y aura une limite, notre analyse tiendra la route. 
Il est certes possible de voir une justification fictionnelle à toute cette progression (le personnage s'améliore pour pouvoir faire face à des menaces de plus en plus féroces), et $c^{\prime}$ est pourquoi nous avons choisi cet aspect comme fil conducteur. Maintenant, nous désirons montrer que cette progression s'effectue selon un système d'ensembles d'actions segmentées, et se regroupant en ensembles plus grands. Nous qualifions ces petits ensembles d'actions de quêtes. Ces dernières peuvent être explicites ou implicites. Une quête explicite est identifiée comme telle par le jeu, énonce ses objectifs, son mode de résolution et les récompenses qui lui sont associées, et génère diverses actions et rencontres. Si l'inscription de ces quêtes au sein de la trame fictionnelle est difficile (d'où notre choix de nous référer à un grand ensemble ludique), toutes sont, individuellement, clairement parties prenantes de la fiction. Les quêtes implicites, quant à elles, sont composées d'objectifs déterminés par le joueur - atteindre un certain niveau, éliminer le dragon tapi au fond d'un donjon - qui ne sont donc pas inscrits dans la fiction. Elles possèdent néanmoins leur propre portrait intentionnel (celui du joueur, naturellement) et sont également génératrices de rencontres et d'actions.

Contrairement à Fallout 3, qui permettait, grâce à son mécanisme de nivellement, d'accomplir les quêtes dans l'ordre voulu tout en préservant la linéarité de la progression ludique, World of Warcraft associe ses quêtes à un niveau de progression ludique, qui sert ainsi de cadre principal. En effet, un personnage de niveau 30 ne peut même pas obtenir les quêtes de haut niveau - et s'il lui est possible de faire celles de niveau beaucoup plus bas, elles ne lui apportent pratiquement rien pour la progression ludique. De cette façon, 
le programme restreint le nombre de possibilités offertes à un moment donné, et dirige systématiquement les personnages vers des zones du monde fictionnel où les quêtes sont compatibles avec leur niveau de puissance. Ainsi, le cadre ludique détermine ainsi celui du récit. C'est ce qui fait qu'on associe souvent l'expression «parc d'amusement» à ce type de jeu: plutôt que de permettre une libre exploration, il encadre fortement la progression. Le monde de Warcraft est sectionné en différentes zones, qui offrent chacune un cadre géographique (pics enneigés, montagnes, déserts) et un cadre ludique, un niveau de puissance suggéré (telle zone accueille les joueurs débutants, telle autre ceux de niveau 80). Certaines zones ne sont accessibles que pour certains joueurs, selon leur allégeance et leur personnage. Passer d'une zone de niveau faible à celle qui la suit directement est généralement aisé et intuitif, et facilité par des transports rapides lorsqu'elles sont éloignées 1'une de l'autre. Ainsi, malgré l'apparente ouverture offerte par l'absence de trame principale du récit, World of Warcraft demeure très linéaire, n'offrant que quelques alternatives à chaque niveau de puissance donnée.

Voici (ci-bas) la carte des différentes zones, chacune étant annotée du niveau suggéré pour les visiter, et liées par la progression naturelle des quêtes. D'une complexité apparente, il s'agit en fait d'une carte très simple, où les possibilités sont plutôt restreintes. 


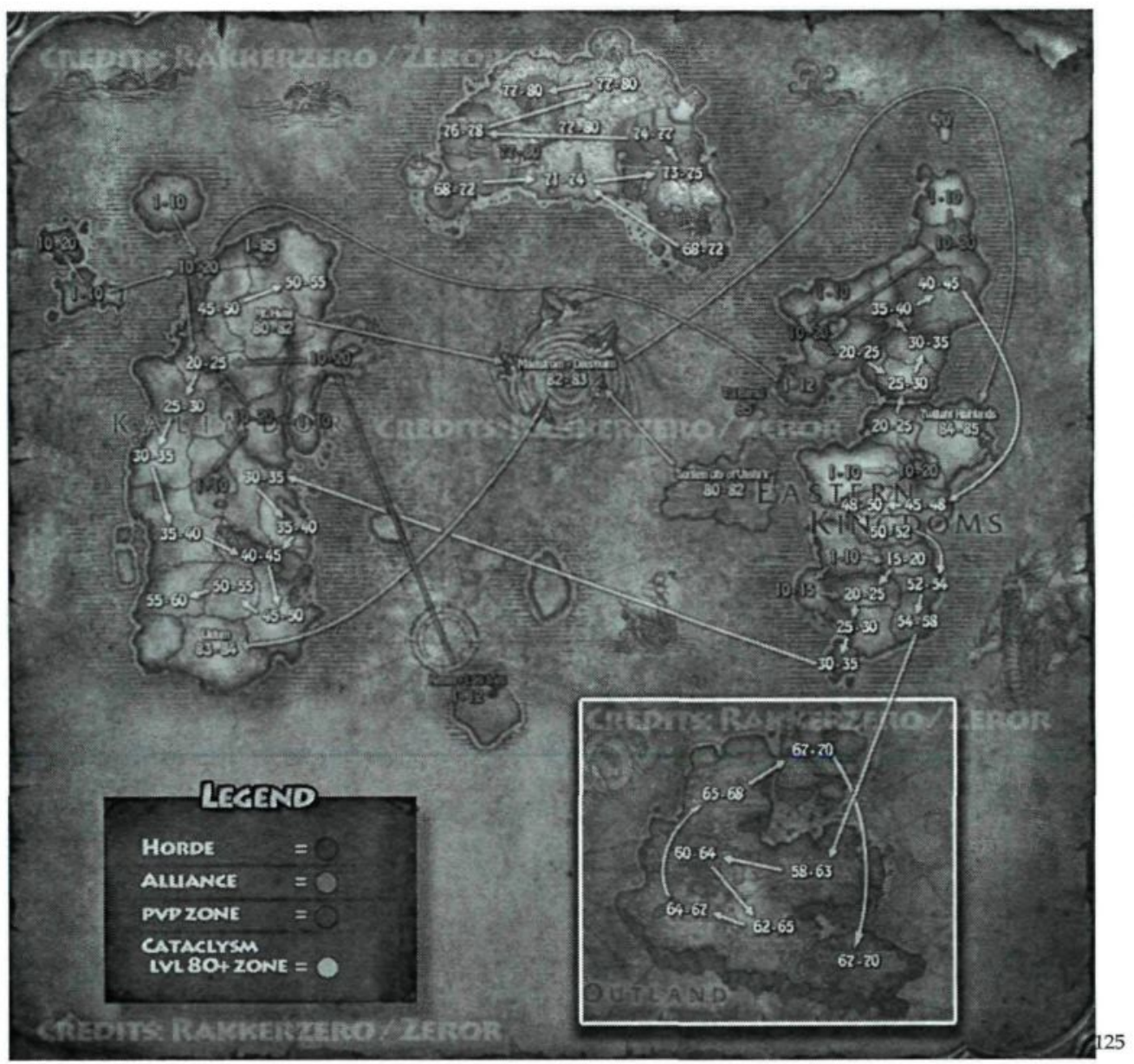

Les quêtes de chaque zone peuvent généralement être regroupées en une ou quelques séquences linéaires, en courts récits. Les situations finales de ces séquences ouvrent généralement sur une raison fictionnelle pour envoyer le personnage dans une zone appropriée de niveau supérieur, et ainsi se poursuit la ronde de la progression de World of Warcraft. À une quête principale unique, qui dirigerait le récit du début à la fin, le jeu substitue différentes séquences, qui peuvent être explorées indépendamment, pourvu que le personnage ait atteint un niveau approprié de puissance. À ces quêtes offertes un peu

125 "World of Warcraft Zone Leveling Map (Level 1-85) Guide", 24 Seven Post (3 janvier 2011). $<\mathrm{http}: / / 24$ sevenpost.com/world-news/world-warcraft-zone-leveling-map-guide/ > (15 septembre 2011) 
partout, s'ajoutent des rencontres indépendantes, quêtes implicites ou ennemis en liberté, qui viennent étoffer les divers récits du jeu.

Les règles ludiques suggèrent fortement une certaine progression et empêchent certains parcours mais, une fois le niveau maximal atteint, le joueur peut envoyer son personnage parcourir les séquences qui lui plaisent. Du point de vue fictionnel, donc, chaque zone représente un récit complet et balisé, et la trame principale fictionnelle de World of Warcraft se perçoit dans le passage d'une séquence à l'autre (relativement) librement, dans la mesure où ces séquences sont accessibles. Nous proposons d'appeler ce type de structure narrative une structure modulaire. Au sein d'une structure modulaire, le joueur peut agencer les différents modules (récits indépendants, quêtes) dans l'ordre qui lui convient. Certaines contraintes existent, comme nous l'avons vu plus tôt, mais ce modèle demeure essentiellement valable pour ce type de jeu. Chaque module devient un récit en soi, dans lequel s'inscrivent les différentes quêtes et rencontres, et l'expérience complète du jeu devient, pour ainsi dire, épisodique.

$S^{\prime}$ il est tout à fait possible pour le joueur d'ignorer complètement les quêtes offertes, et les progressions suggérées par le jeu, World of Warcraft multiplie les efforts pour rendre cette approche inefficiente. On en voit plusieurs exemples : des ennemis de trop haut niveau sont intouchables, alors que ceux de bas niveau ne donnent aucune expérience ; certaines zones fermées ne peuvent même pas être abordées si le personnage n'a pas le niveau requis; les récompenses offertes par les quêtes sont exponentiellement plus puissantes que 
celles qu'il est possible d'obtenir en enchaînant les rencontres. C'est encore là la progression ludique qui gère le tout et, en rendant certaines façons de jouer peu efficaces, World of Warcraft s'assure de maintenir les joueurs sur les rails, et ainsi d'offrir à chacun une expérience contrôlée et similaire. Son apparente ouverture n'est ainsi, en majeure partie, qu'une illusion - sa non-linéarité est trompeuse, et l'expérience du jeu comme de la fiction y demeure essentiellement séquentielle et ordonnée, bien que fragmentée.

\section{Valkyria Chronicles}

Valkyria Chronicles, fidèle à la tradition des jeux de stratégie japonais, ${ }^{126}$ présente une structure du récit proche de celle des médias traditionnels, divisée en chapitres, puis en scènes interactives (les missions) et en scènes programmées (les animations). Quoique s'écartant très peu des modèles familiers, ce jeu présente néanmoins plusieurs éléments fascinants.

Il est ainsi aisé de repérer la structure narrative de Valkyria Chronicles : le jeu se charge luimême de diviser en différents sous-groupes ses principaux ensembles d'actions. Le récit est présenté comme issu d'un livre, qui raconte les événements ayant mené à l'ascension du personnage principal de simple milicien à figure emblématique d'une vaste guerre rappelant (avec quelques éléments fantastiques) vaguement la Seconde Guerre mondiale. Ce livre se segmente en différents chapitres, eux-mêmes découpés en scènes, qui doivent

\footnotetext{
126 Par exemple, Final Fantasy Tactics (SquareSoft - Final Fantasy Tactics, SCEA, 1998), Tactics Ogre (Quest Tactics Ogre; Let Us Cling Together, Atlus, 1995), Vandal Hearts (Konami Computer Entertainment Tokyo Vandal Hearts, Konami, 1996), Disgaea (Nippon Ichi Software - Disgaea: Hour of Darkness, Atlus, 2003), et de nombreux autres.
} 
être jouées dans l'ordre, laissant du coup très peu de latitude au joueur quant à l'agencement des situations intermédiaires de la trame narrative principale. La division est déjà faite, tout comme la sélection des actions à accomplir.

Il existe cependant quelques nuances. Ainsi, si la structure principale est fixe, elle peut également être bonifiée de scènes optionnelles. Par exemple, dans le chapitre représenté sur l'image ci-dessous, il est possible de repérer différentes scènes obligatoires, reconnaissables au chiffre arboré dans le coin inférieur droit de leur image. Sur la page de gauche une image porte, au lieu d'un chiffre, un astérisque, ce qui l'identifie comme scène optionnelle.

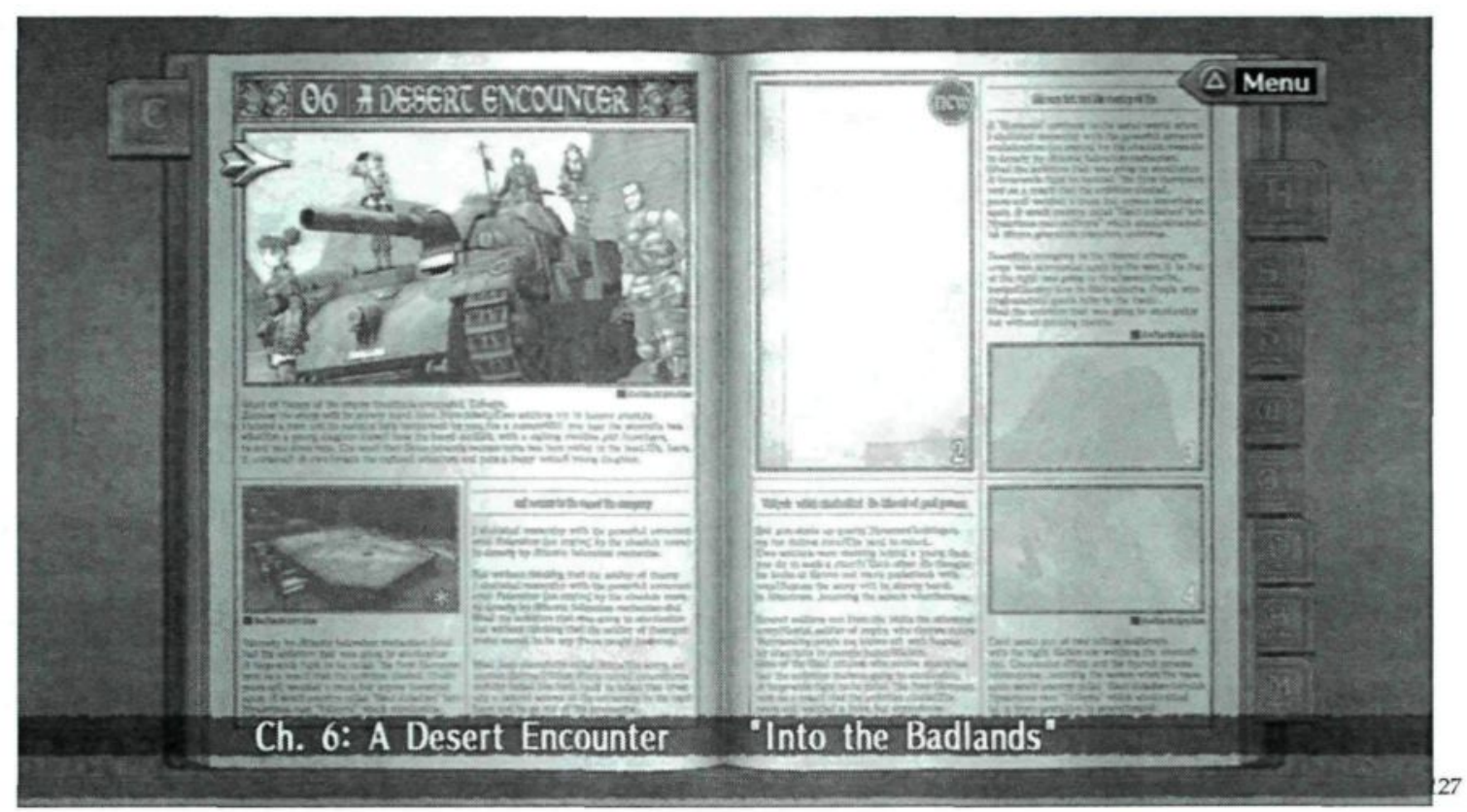

Il est ainsi possible pour le joueur de ne pas la visionner - mais non de faire en sorte qu' elle ne se produise pas. L'une d'elles, par exemple, montre une réunion entre les dirigeants

${ }_{127}$ Sega - Valkyria Chronicles. (A Desert Encounter) 
d'un empire voisin, et éclaire un peu mieux leurs motivations. Qu'on visionne ou non cette scène, ces personnages se réunissent et prennent les mêmes décisions - l'événement n'a simplement pas été représenté par le jeu. Les scènes optionnelles de Valkyria Chronicles, en effet, sont un complément à la quête principale, au sein de laquelle elles ne s'inscrivent pas nécessairement - ce sont donc, dans le sens le plus pur du terme, des situations narratives subordonnées. Elles se consacrent plutôt à développer certains personnages ou intrigues qui ne sont pas au cœur des événements. Ainsi, le choix du joueur ne se situe pas au niveau de la structure narrative, mais est plutôt de l'ordre du « droit de sauter des pages »128 proposé par Pennac.

Chaque scène est elle-même composée de plusieurs sous-ensembles, et c'est là que la capacité d'interaction vidéoludique entre en jeu avec le récit. Il y a deux types de scènes, que nous avons mentionnées brièvement en début de section: les animations et les missions. Les premières ne demandent aucune contribution du joueur quant à leur déroulement: il s'agit d'événements qui ont un impact dans le déroulement de l'histoire, et il suffit de les visionner pour passer à la scène suivante. Les missions, quant à elles, ressemblent à bien des égards à notre prototype de quête. Elles établissent un objectif clair (éliminer tous les ennemis, s'enfuir sain et sauf) donnant au personnage une motivation sensée et récompensent le joueur par une progression ludique, déployant ainsi un schème interactif complet. Le joueur doit ensuite tenter d'atteindre $1^{\prime}$ objectif en prenant le contrôle

${ }^{128}$ Daniel Pennac - Comme un roman, Gallimard, 1992, p.145. 
tour à tour des différents combattants qu'il choisit de déployer. ${ }^{129}$ Chaque « tour » de jeu (moment où le joueur prend le contrôle d'un personnage) constitue en soi une action, qui doit s'effectuer en considérant les ressources disponibles, matérielles, humaines ou temporelles. En autant que la somme des actions accomplies mène à l'atteinte de l'objectif global de la mission, le joueur est libre de décider quelles actions accomplissent ses personnages, et dans quel ordre.

Pour compliquer les choses, aux forces contrôlées par le joueur s'opposent presque systématiquement des forces ennemies, dont les actions sont déterminées par le programme. Là encore, on y voit un enchaînement d'actions par le joueur et de réactions par le programme, créant une panoplie de situations dramatiques. Ainsi, le récit produit au sein des missions est le résultat des actions entreprises par le joueur pour résoudre le problème posé par les systèmes de règles ludiques et fictionnelles (les objectifs peuvent être dictés par l'un ou l'autre) confrontées à celles déterminées par le programme. Toutefois, il ne s'agit déjà là que de situations intermédiaires : les éléments ici mentionnés $\mathrm{n}^{\prime}$ affectent pas le cours des événements, ils ne font que s'inscrire dans le sillage d'une action plus grande qui, elle, est hors du contrôle du joueur.

Chaque «tour » de jeu est, pour revenir à notre modèle, l'équivalent d'une rencontre. Il $\mathrm{s}^{\prime}$ agit à chaque fois, au minimum, d'un conflit entre les actions nécessaires pour atteindre I'objectif et les ressources disponibles. Le rôle tenu par le joueur dans Valkyria Chronicles,

129 De nombreuses ramifications stratégiques s'ajoutent évidemment à cette simplification, mais nous ne jugeons pas nécessaire de les détailler ici. 
par rapport à la structure du récit, se limite donc principalement à sélectionner et agencer différentes rencontres à l'intérieur des quêtes, sous forme de missions.

Somme toute, et sans grande surprise, Valkyria Chronicles se laisse lire plutôt que jouer. II s'agit d'un exemple parfait d'un jeu dirigiste et linéaire, qui confirme la thèse des ludologistes pour qui récit et jeu ne peuvent se vivre que séparément pour être efficaces. Ce n'est pas là quelque chose de négatif - en minimisant les écarts possibles, le jeu s'assure de la pertinence et de la cohérence de sa trame principale, qui n'en est que mieux rythmée. Certaines scènes sont optionnelles, pour les plus curieux, et il est même possible de « rejouer » des missions passées, comme il est possible de relire certains passages d'un roman, mais cela n’a aucun effet sur la progression du récit.

On avance ainsi toujours dans la même direction, et les variations possibles ne font pas diverger le cours du récit autrement qu'en le retardant. Le personnage suit ainsi nécessairement une séquence de situations directrices, avec la possibilité d'explorer une sélection de situations subordonnées. Valkyria Chronicles est en somme un jeu électronique qui réifie le prototype de l'action, du récit et des enchaînements proposé par Gervais. $C^{\prime}$ est donc ici la linéarité du récit qui remporte le bras de fer entre fiction et jeu.

\section{Conclusion}

Les possibilités offertes par le jeu vidéo en fait de structure narrative sont énormes. Ce ne sont pas nécessairement des modes de fonctionnement possibles uniquement dans le 
vidéoludique, mais le fait est qu'on peut les retrouver dans les jeux les plus populaires, et non seulement dans les jeux expérimentaux. Nos trois œuvres nous ont fourni trois structures différentes: la structure en arborescence dirigée (Fallout 3), la structure modulaire (World of Warcraft) et la structure linéaire, avec compléments possibles (Valkyria Chronicles). L'une n'est pas meilleure que l'autre : à chaque jeu sa structure idéale.

Le joueur occupe une place bien différente dans les trois jeux qui ont retenu notre attention. Celui de Fallout 3 peut accomplir une grande diversité d'actions pour parvenir à ses fins, et ses choix, qui définissent pleinement le déroulement des actions, s'inscrivent néanmoins dans une même trame principale. Ce jeu parvient ainsi à bien représenter l'idée selon laquelle il existe de nombreuses façons de raconter la même histoire. World of Warcraft, qui semble décousu dans l'ensemble, présente à son joueur une progression ludique à toutes fins pratiques linéaire. Cependant, en détachant ses différentes séquences l'une de l'autre, il permet sur le plan fictionnel d'agencer librement ses différents modules. Et Valkyria Chronicles, quant à lui, rend explicite sa structure narrative linéaire, ne laissant que la progression ludique entre les mains du joueur. Il ne sacrifie ainsi pas l'efficacité du récit, qui peut être rythmé de façon efficace. 


\section{CHAPITRE III}

\section{UN MONDE DE POSSIBILITÉS - LECTURE VIDÉOLUDIQUE}

Dans notre section précédente, nous avons examiné l'architecture narrative des jeux vidéo, la façon dont ils organisent leurs différents éléments narratifs. Il nous semble maintenant nécessaire de centrer notre réflexion sur la réception de ces éléments par le joueur, et sur l'importance de ce processus dans l'expérience narrative vidéoludique. Penser le jeu vidéo en fonction du joueur, dès les premiers travaux sérieux de "game studies », est apparu comme l'approche la plus logique. Cherchant à cerner l'unicité de ce mode d'expression, des penseurs comme Juul130 ou Aarseth ${ }^{131}$ ont identifié le rôle particulier qu'y est amené à prendre le joueur : une place centrale qui remettait en question l'idée même de narration (pour le premier) ou qui venait conférer (pour le second) un statut ontologiquement différent au vidéoludique, en tant qu' ouvre cybertextuelle.

Nous développerons notre modèle de la lecture vidéoludique en empruntant d'abord à Aarseth cette notion de cybertexte - des textes sur support informatique. Suivant sa pensée, ces derniers profitent du support informatique pour exiger de leur usager un effort non trivial, par opposition aux textes traditionnels, dont la lecture ne demanderait aucun effort en dehors du mouvement des yeux ou du fait de tourner les pages. Cet effort non trivial n'est pas nécessairement un effort physique; il s'agit plutôt de "calculs »

130 Jesper Juul - A Clash between Gante and Narrative.

131 Espen Aarseth - Cybertext; Perspectives on Ergodic Literature. 
devant être accomplis pour produire les éléments narratifs de l'œuvre, calculs pouvant être accomplis aussi bien par un programme informatique (une intelligence artificielle qui ajuste son comportement à une situation changeante) que par l'usager lui-même (en déterminant, par exemple, le meilleur chemin à suivre pour résoudre une énigme). Bien $q^{\prime}$ 'Aarseth parle principalement de textes, on peut étendre, comme l'ont fait plusieurs ludologistes, son approche à des œuvres qui ne sont pas purement textuelles, créant ainsi une distinction entre les médias ou œuvres demandant cet effort de calcul, et les autres. Les jeux électroniques seraient donc foncièrement différents des médias traditionnels, comme le cinéma ou le roman, parce qu'ils exigent un effort réel de la part du joueur, et parce qu'ils sont gérés par un programme informatique dont les calculs sont producteurs de ses éléments narratifs.

Plus encore que d'exiger un effort non trivial, le cybertexte, pour Aarseth, est un texte dynamique, au sens où, au regard des éléments qui lui sont transmis par l'usager (éléments déterminés par les calculs exigés), le cybertexte peut modifier son texte, ou encore la configuration de ses éléments textuels. ${ }^{132}$ Si nous ne sommes pas d'accord avec toute sa pensée (la notion d'effort de lecture y est particulièrement étrange), nous considérons tout de même son modèle d'analyse pertinent, et il nous servira par la suite. Suivons celui-ci, résumé à l'intérieur de ce graphique :

132 Ibid. p.62-65. 


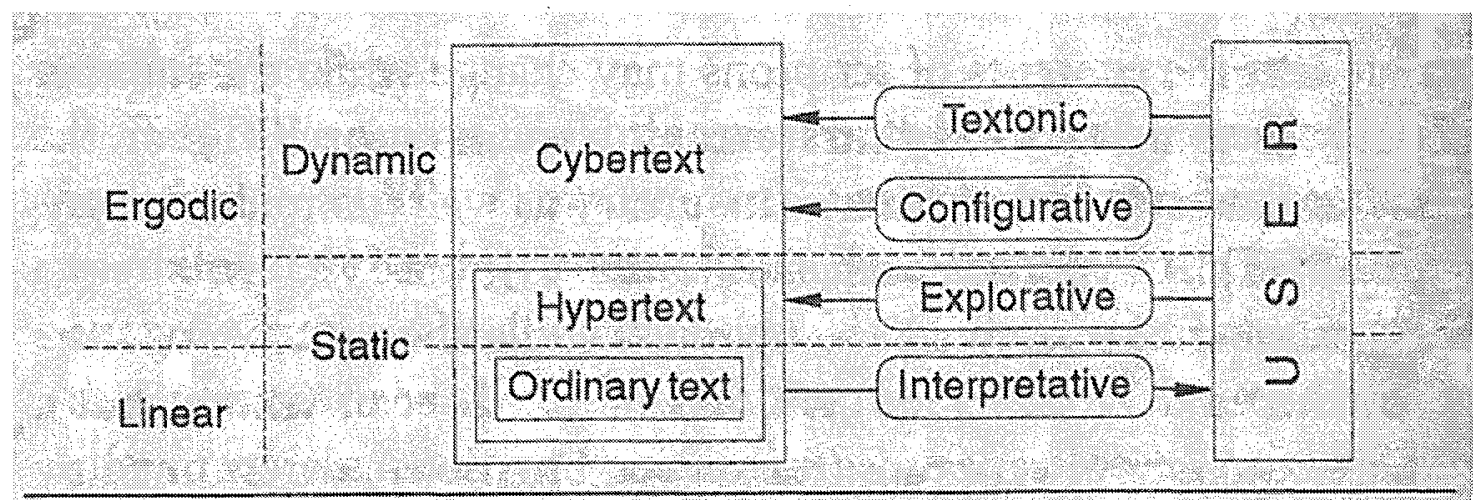

Figure 3.1. User Functions and Their Relation to Other Concepts

Détaillons d'abord les trois types de textes présents, avant d'explorer les rapports à l'usager qu'ils supposent. Le texte ordinaire est ainsi considéré comme statique et linéaire : statique parce que d'une lecture à l'autre, il s'agit du même texte, et linéaire parce qu'il ne peut être parcouru que d'une façon. On pourrait classer dans cette catégorie maints romans classiques (ceux de Zola ou de Balzac) ou films populaires.

L'hypertexte, également statique, se différencie par son côté ergodique, c'est-à-dire qu'il exige un effort de la part de l'usager pour le parcourir, au sens où l'usager doit choisir (à l'aide de calculs) la suite de son parcours. Dans ce cas, on le dit statique parce que chaque élément du texte s'y retrouve au même endroit, et est accessible de façon systématique - le texte lui-même ne change pas, seul le parcours change. En plus des différents textes en hyperliens disponibles sur Internet, nous pouvons aussi ajouter à cette catégorie les livresjeu du type «livres dont vous êtes le héros » dont la représentation est statique, mais le parcours ergodique.

133 Ibid. p.64. 
Le cybertexte à proprement parler pousse la différence plus loin, car il s'agit d'un texte essentiellement dynamique, au sens où les éléments textuels et leur agencement peuvent être variables. Aarseth donne le jeu électronique Adventure ${ }^{134}$ comme exemple d'une œuvre dont les éléments textuels ont un nombre variable de combinaisons possibles ou de programmes bonifiés par la contribution d'usagers, et dont le nombre d'éléments textuels n'est pas même connu ${ }^{135}$.

Lès trois types de textes appellent également des rapports différents à l'usager, comme le montre la partie droite du schéma. L'interprétation, seule fonction présente dans tous les textes, consiste en une série de «décisions [...] concernant sa signification. »136 L'information circule du texte vers l'usager, qui doit ensuite l'interpréter - pas de retour possible. L'exploration est présente lorsqu'un lecteur doit «prendre des décisions stratégiques face à des chemins alternatifs et, dans le cas des jeux d'aventure, des actions alternatives. »137 La configuration et la textonique concernent les éléments textuels : une relation de configuration demande à l'usager de configurer son texte en agençant ses éléments, ou en modifiant ses variables; un rapport textonique suppose à l'usager la capacité de «changer le texte en ajoutant [sa] propre écriture ou programmation. »138 Dans les trois derniers cas, l'information circule de l'usager vers le texte - c'est ce dernier

\footnotetext{
134 Atari-Adventure, Atari, 1978.

135 Espen Aarseth - Cybertext; Perspectives on Ergodic Literature., p.63.

136 Ibid. p.64. (Nous traduisons)

137 Ibid. (Nous traduisons) (Nous soulignons)

138 Ibid. (Nous traduisons)
} 
qui doit prendre en considération les choix de l'usager pour offrir le bon enchaînement (exploration) ou se reconfigurer (configuration et textonique). ${ }^{139}$

Il faut noter qu'Aarseth propose un jeu d'aventure comme exemple d'un cybertexte, avant de classifier ce type d'œuvre fermement dans la catégorie de l'hypertexte. Il est en effet des jeux plus ou moins permissifs quant au rôle tenu par l'usager. À ce propos, nous désirons proposer de considérer le jeu électronique non pas systématiquement comme un cybertexte, mais plutôt comme un cybertexte potentiel. En effet, le support informatique, en permettant à l'œuvre d'analyser les informations envoyées par l'usager, rend possibles les différentes fonctions du cybertexte (exploration, configuration et textonique), mais il ne les rend pas nécessaires, et c'est pourquoi, dans notre analyse, nous tenterons d'observer vers quelle catégorie chacun des jeux à l'étude tend : se rapproche-t-il d'un texte ordinaire, d'un hypertexte ou d'un cybertexte?

Les différences fondamentales entre la lecture d'un cybertexte et d'un texte " ordinaire » apparaissent maintenant de façon flagrante : la communication entre le second et le lecteur ne se fait qu'à sens unique, d'où le terme «effort» choisi par Aarseth, qui présente l'interprétation comme un rapport passif, ne demandant pas de réel « effort ». Même si sa classification nous appert efficace, nous ne sommes pas d'accord avec lui sur ce point: comme Eco, qui parle de « coopération interprétative ${ }^{140}$, nous pensons l'interprétation

\footnotetext{
${ }^{139}$ Cette possibilité de communication est d'ailleurs à la base du concept de narration dialogique, développée dans Samuel Archibald - Le texte et la teclnique : la lecture à l'heure des nouveaux médias, p.169-170. 140 Umberto Eco - Lector in fabula; Le rôle du lecteur, p.3.
} 
comme un travail sur le texte. C'est pourquoi nous désirons faire appel à une approche différente de l'interprétation, qui demeure essentielle pour comprendre le travail de lecture de tout texte, puisqu'il s'agit de la seule fonction de l'usager qui est nécessairement présente. Pour ce faire, nous emprunterons à l'approche systémique développée par Eco dans Lector in Fabula, ainsi que, plus récemment, par Ryan dans Possible Worlds, Artificial Intelligence and Narrative Theory.

Eco, d'abord, comme nous l'avons vu dans notre premier chapitre, présente la fiction comme un ensemble de propriétés - ce que nous avons renommé un ensemble de règles fictionnelles. Ces règles déterminent ce qui est, et comment cela est, construisant 1'expérience du récit et de sa lecture. Le texte, ou la représentation, de ce système et de son évolution est par nature « incomplet » $^{141}$ et le travail de lecture, en ce cas, consiste à le compléter - c'est là qu'intervient la lecture interprétative. La lecture est un travail qui donne corps au texte, qui l'actualise, et elle est principalement constituée d'inférences qui viennent contribuer à enrichir le texte lui-même, et génèrent l'expérience de la lecture.

Un lecteur n'est pas ainsi récepteur passif - il est actif, cherche à reconstruire la fiction, et cherche également à prévoir et anticiper les développements possibles du récit. Ce travail d'anticipation consiste en « échappées hors du texte "142 qu'Eco appelle des promenades inférentielles. Par là, Eco suggère qu'en plus de se baser sur la fiction elle-même, les inférences du lecteur sont également intertextuelles, font appel à une compréhension du

141 Umberto Eco - Lector in fabula; Le rôle du lecteur, p.62.

142 Ibid. p.151. 
genre, du monde et de l'action. Intervient donc dans la lecture tout un bagage du lecteur, qui contribue à l'expérience du texte. Sachant cela, il est possible pour nombre d'œuvres de manipuler ce processus de lecture, en anticipant les constructions possibles du lecteur. Autrement dit, on considère le texte comme essentiellement interactif : $l^{\prime}$ interaction se joue entre auteur et lecteur, chacun tentant de cerner, par le biais des Lecteur et Auteur modèles, les stratégies de l'autre.

Le modèle des mondes possibles vient offrir une vision systémique de la façon dont sont construites ces différentes prévisions, et donc du fonctionnement du travail de lecture. À partir d'un état de la fiction, d'un ensemble donné de propriétés, les différents développements potentiels prévus peuvent être perçus comme des «mondes possibles. » Ces derniers sont ainsi des constructions mentales, basées sur les inférences du lecteur, ainsi que sur sa compréhension des propriétés fictionnelles. Tous les mondes ne $\mathrm{s}^{\prime}$ équivalent pas, cependant : ils ne sont pas tous nécessairement accessibles. Eco dit qu'un monde donné est accessible à partir d'un monde d'origine « si, à partir de la structure [du monde d'origine], il est possible de générer, par la manipulation des rapports entre individus et propriétés la structure de [celui-ci]. »143 Cette particularité ne nous suffit toutefois pas - si la notion d'accessibilité permet de circonscrire les possibles développements, elle ne nous permet pas de les hiérarchiser. Plutôt que de parler d'accessibilité des modes possibles, nous préférons parler de rentabilité des inférences du lecteur face à la construction de mondes.

143 Ibid. p.186. 
Il s'agit, somme toute, d'un concept assez simple: les inférences de lecture se doivent d'être rentables, donc de présenter un écart minimal face à la réalité textuelle, et au réseau intertextuel du lecteur, exploré par ses promenades inférentielles. Face à l'affirmation «Roger téléphone à Julie », un lecteur qui sait que Roger possède un téléphone portable construit plus spontanément l'idée que Roger emploie celui-ci, et aura du mal à défendre l'idée qu'il déambule plutôt dans les rues pour trouver un téléphone public qui fonctionne afin de faire son appel : la première idée est tout simplement plus rentable. Certes, la seconde est possible (il s'agit d'un monde accessible), et pourrait même se révéler la bonne, mais elle demande un plus grand écart avec la logique textuelle. On construit des mondes plausibles, ne prévoyant, en général, que des écarts minimaux avec les propriétés déjà établies. Les facteurs selon lesquels opère le jugement de rentabilité seront explorés plus en détail avec la pensée de Ryan.

La notion de rentabilité est chère à la lecture interprétative, puisqu'elle permet de comprendre le travail effectué par le lecteur, et les impératifs auxquels il obéit. De même, elle permet de mieux comprendre les démarches d'écriture, qui peuvent utiliser les a priori du lecteur pour créer des effets de surprise, voire un monde fictionnel intentionnellement déstabilisant, allant à l'encontre d'une logique de rentabilité conventionnelle. Pour nous, cependant, elle joue plus encore un rôle également capital dans le fonctionnement des autres types de lecture proposés par Aarseth : exploration, configuration et textonique. Dans une lecture-exploration, par exemple, il est des chemins qui se révèlent plus rentables que d'autres, pour plusieurs raisons : ils s'attardent sur des éléments cruciaux du 
récit plutôt que périphériques, par exemple. Il en va de même pour les fonctions de lecture associées au cybertexte : l'agencement des éléments textuels ou la configuration des séquences obéissent à une certaine rentabilité, qui cherche à minimiser l'écart avec la réalité textuelle première, et à construire un sens plausible - dans l'œil du lecteur.

C'est pourquoi nous postulons qu'une approche par inférences, émanant de la théorie des mondes possibles, couplée à la notion de rentabilité, peut nous offrir une meilleure compréhension des différents travaux de lecture nécessaires dans le cas du cybertexte, donc possibles dans le jeu électronique. Avant de détailler cette approche, à l'aide de la pensée de Ryan, nous croyons toutefois nécessaire de retourner une dernière fois à celle d'Aarseth, qui explore un point crucial de la relation entre le lecteur et le cybertexte: l'interaction nécessaire entre les deux. Plus encore, Aarseth s'attache en particulier aux cybertextes de forme «jeux de rôle» pour explorer la manière dont y fonctionne la communication entre lecteur et texte - ce qui nous ramène directement aux jeux électroniques qui nous occupent ici.

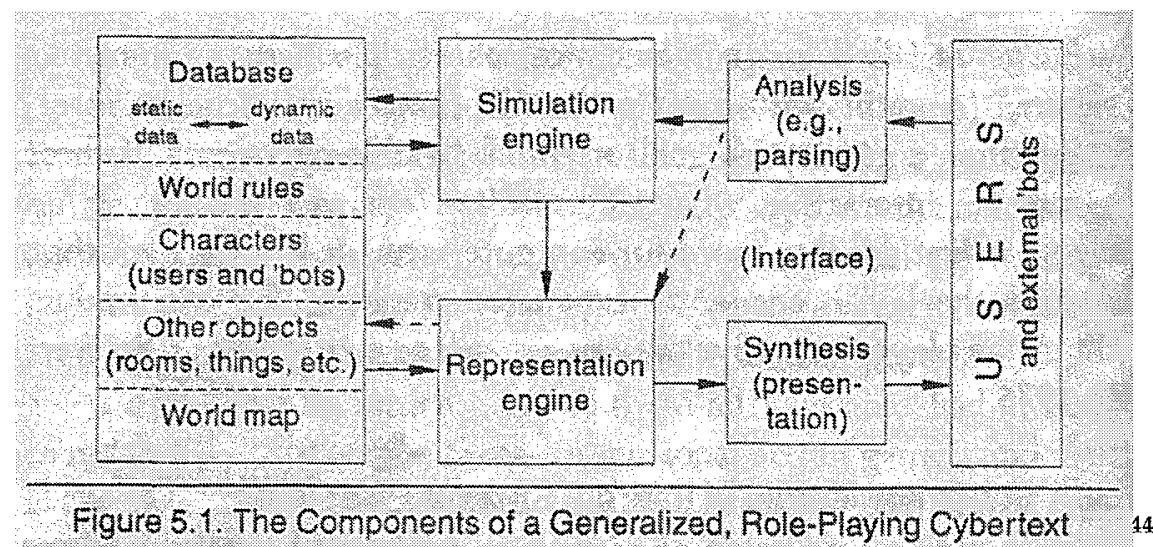

144 Espen Aarseth - Cybertext; Perspectives on Ergodic Literature, p.104. 
L'essentiel pour nous est ici la relation circulaire des éléments du cybertexte. De la banque de données (les règles du monde, à la fois règles de fiction et de jeu, sujet de notre premier chapitre) est issue une représentation (le récit, sujet de notre deuxième chapitre) accomplie par un « moteur »- le programme, en quelque sorte. Ce récit est lu par l'usager (avec tout ce que la lecture interprétative implique) qui peut alors communiquer avec le programme, à travers une simulation (appuyer sur tel bouton simule telle action, par exemple). Cette communication est représentée et surtout, analysée par la banque de données - qui peut modifier en conséquence sa représentation.

Ainsi, le jeu électronique est en constante communication avec le joueur et s'adapte sans relâche aux éléments communiqués par celui-ci. L'usager ne peut exercer ses différentes opérations de lecture qu'à travers cette relation circulaire. Cette relation est donc nécessaire à une lecture cybertextuelle : lectures configurative et textonique ne peuvent se faire que dans une interaction dynamique, où l'œuvre lue est en mesure de réagir aux éléments communiqués par son usager. Le choix des éléments à communiquer est nécessairement influencé à son tour par une lecture interprétative des représentations offertes par l'œuvre. D'un côté, donc, l'usager lit, choisit ce qu'il communique, et de l'autre le programme analyse et répond, obéissant à une logique procédurale, par une nouvelle représentation.

Revenons maintenant à la notion de rentabilité et à sa pertinence pour les différents types de lecture. Les interprétations de l'usager d'un cybertexte obéissent sans contredit à une 
logique de rentabilité, de par leur processus synthétique - mais les éléments qu'il choisit de communiquer le font également. Afin d'obtenir la configuration souhaitée, l'usager doit communiquer les bons éléments, ceux qui sont, en bref, les plus rentables. Le processus de communication obéit sensiblement à la même logique que celui de l'interprétation: il s'agit, dans les deux cas, de lecture, au sens le plus large, et ses procédés (inférences, prévisions, rentabilité) sont similaires. Les décisions prises par programme informatique, quant à elles, obéissent à une logique procédurale, étant déterminées par une intelligence artificielle qui respecte également une forme de rentabilité.

Si elle n'utilise pas le terme de rentabilité, Ryan développe néanmoins une vision s'en rapprochant dans son Possible Worlds, Artificial Intelligence and Narrative Theory. ${ }^{145}$ Dans cet ouvrage, Ryan détaille une approche systémique du récit, basée sur l'approche par mondes possibles. Elle tente principalement de montrer comment cette approche permet de mieux comprendre à la fois la dynamique de l'action à l'intérieur même de la fiction, et le fonctionnement de l'intelligence artificielle, en tant qu'agent d'un récit. Pour nos besoins, nous avons principalement retenu son chapitre « The Modal Structure of Narrative Universes », consacré à l'élaboration d'une logique de l'action fictionnelle en harmonie avec l'approche par mondes possibles.

145 Voir dans Marie-Laure Ryan - Possible Worlds, Artificial Intelligence, and Narrative Theory, p.109-147. 
Elle y propose, à l'instar de Gervais, de considérer l'action comme l'élément central du récit. Sa contribution première se situe cependant sur le plan du portrait intentionnel : Ryan développe une pensée de l'intention qui est inspirée de l'approche par mondes possibles. Il nous faut ici rappeler qu'en deuxième chapitre, nous avons proposé de voir, dans le jeu électronique, les actions d'un agent contrôlé par le joueur comme obéissant à deux portraits intentionnels : celui du personnage et celui du joueur. En prenant la vision de Ryan, qui s'applique à des agents fictionnels, et en l'appliquant plutôt à l'agent-joueur, nous pensons pouvoir dégager une pensée de l'intention du joueur, construite par les représentations du jeu, et qui peut lui être communiquée. Autrement dit, nous allons maintenant tenter de comprendre la lecture vidéoludique comme la construction de l'intention et de l'action du joueur, obéissant à la même logique par mondes possibles que présente Ryan pour comprendre l'intention et l'action du personnage.

Ryan postule d'abord qu'il existe un monde textuel actuel, « une succession de différents états et événements [...] comprenant un ensemble de règles générales qui déterminent l'étendue des développements futurs possibles du récit à partir de la situation présente. »146 Ce monde, qui existe en tant que référent extérieur à la représentation des différents éléments textuels, n'est pas nécessairement en adéquation avec la vision qu'en

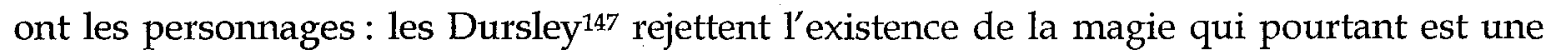
composante essentielle du monde qu'ils habitent. Il n'existe donc, pour le personnage, que

\footnotetext{
${ }_{146}$ Marie-Laure Ryan - Possible Worlds, Artificial Intelligence, and Narrative Theory, p.113. (Nous traduisons) 147 Il s'agit ici d'une référence à une célèbre famille de Moldus appartenant à l'univers de Joanne K. Rowling Harry Potter and the Philosopher's Stone, Bloomsbury, Londres, 1997.
} 
l'image qu'il se fait du monde textuel actuel, que nous appellerons monde perçu. À partir des différents états de ce monde perçu, il leur est possible d'inférer et d'évaluer divers dénouements potentiels, construisant ce que Ryan nomme des mondes privés appartenant à un «domaine prospectif ${ }^{148}$ - ce faisant, ils s'affairent à déterminer les mondes accessibles, et à déterminer leur rentabilité.

Nous avons discuté précédemment de la notion de rentabilité, où nous avancions que les mondes accessibles ne sont pas tous égaux - certains sont plus probables, et il revient au lecteur d'établir, selon sa compréhension de la fiction, desquels il s'agit. Ryan, quant à elle, avance que pour le personnage, qui doit utiliser sa compréhension du monde afin de déterminer quelle(s) action(s) entreprendre, la probabilité d'un monde n'est qu'un des facteurs déterminant la rentabilité des mondes. Le processus selon lequel les personnages déterminent la rentabilité des mondes s'opère plutôt comme un système de classification et de notation : à chaque monde accessible sont attribuées par le personnage trois valeurs prospectives, selon trois axes, qu'il doit jauger et prioriser afin de déterminer vers quel monde il veut voir son monde perçu évoluer (son intention), et quoi faire pour l'y amener (son action). Ces trois axes sont l'axe de la probabilité, l'axe de la désirabilité et l'axe de la moralité. 149

Sur l'axe de la probabilité, les divers dénouements possibles sont classés selon l'écart qu'ils présentent par rapport au monde perçu. Plus l'écart est grand, moins le monde est

148 Marie-Laure Ryan - Possible Worlds, Artificial Intelligence, and Narrative Theory, p.116. (Nous traduisons) ${ }^{149} \mathrm{Ce}$ ne sont pas là les termes exacts employés par Ryan, ni la lettre de sa pensée : nous prenons la liberté de développer notre propre vision, qui demeure fortement inspiré par les travaux de celle-ci. 
probable. Les personnages peuvent avoir une tolérance très différente à la probabilité : l'un, cynique, peut refuser d'agir même si peu lui est demandé pour influer le cours des événements, tandis que l'autre, idéaliste, peut faire tout en son pouvoir pour s'éloigner d'un monde hautement probable, défiant toute logique. La perception de la probabilité, également, est subjective : étant basée sur une perception du monde, elle peut évidemment être erronée.

L'axe de la désirabilité concerne l'attribution à chaque développement (ou plutôt, chaque élément d'un développement) $d^{\prime}$ une valeur de «bien, mauvais ou neutre. »150 Il ne s'agit pas ici de catégories simples, binaires : ce sont plutôt « les pôles d'un continuum »151 qui peut donc présenter une organisation des évolutions du monde de la plus désirable à la moins désirable. La notion de désir est également très complexe, un personnage pouvant très bien désirer différentes choses à différents niveaux de conscience, et pouvant se préoccuper de ce qui est bien non seulement pour lui, mais pour d'autres auxquels il accorde divers degrés d'importance. C'est pourquoi un personnage peut très bien se sacrifier pour le bien' d'un plus grand nombre, bien qu'il ne désire à aucun niveau mourir.

Du point de vue de la moralité, Ryan distingue deux sous-catégories qui en définissent l'axe : la moralité externe (les règles sociales) et la moralité interne (les principes moraux). Ces éléments se combinent en un « système d'engagements et d'interdits »152 qui permet

\footnotetext{
150 lbid. p.117. (Nous traduisons)

151 Ibid. p.118. (Nous traduisons)

152 Ibid. p.116. (Nous traduisons)
} 
au personnage de définir son intention et ses actions comme permises, obligatoires ou interdites. L'axe de la moralité concerne les actions du personnage, et c'est donc en fonction des actions requises par les différents mondes accessibles que ces derniers se voient attribuer une valeur morale. Comme pour l'axe de la désirabilité, l'axe de la moralité peut se révéler des plus complexes, certaines actions étant perçues comme morales à un degré, mais pas à un autre. Chacun peut également attribuer une importance variable aux différents impératifs moraux : un hors-la-loi comme Robin des Bois obéit à ses principes moraux en violant sciemment les règles sociales par exemple.

Une fois ces trois axes considérés, le personnage est à même de déterminer l'action qu'il souhaite entreprendre. L'action est alors dictée par sa compréhension du monde fictionnel (monde perçu), sa perception de son évolution probable (axe de probabilité), la valeur qu'il accorde aux développements possibles (axe de désirabilité) et son rapport aux règles sociales et principes moraux (axe de moralité). Le personnage doit alors déterminer la façon dont il anticipe que son monde perçu évoluera s'il n'agit pas (ce que nous appellerons le monde anticipé) et de là, déterminer quelle(s) action(s) entreprendre pour l'amener au dénouement le plus souhaitable en tenant compte des trois axes - ce monde, nous l'appellerons le monde souhaité.

De là nous voyons se profiler la dynamique gérant l'intention et l'action du personnage. L'essentiel en est contenu dans le conflit : d'abord dans le conflit entre son monde souhaité et son monde anticipé, mais aussi dans le conflit interne représenté par l'inadéquation 
entre les différents axes d'évaluation des mondes accessibles. Lorsqu'un dénouement s'avère le plus probable, désirable pour tous et moral, il n'y a pas de réelle dynamique c'est lorsque le personnage doit choisir, pour établir son monde souhaité, entre des développements, par exemple, improbables mais hautement désirables, moraux mais indésirables, probables mais amoraux, qu'une réelle dynamique s'installe. Pour parler de rentabilité, en se référant au modèle de Ryan, il faut ainsi prendre en considération une multitude d'éléments susceptibles de rendre rentables certaines actions. Il s'agit donc d'une façon d'exprimer les conflits internes qui permettent une compréhension complexe de l'intention et de l'action.

Ryan ajoute à cela une couche de complexité en détaillant une logique du conflit externe, une forme de «situation dramatique ${ }^{153}$ qui détaille le fonctionnement de l'action de plusieurs personnages présentant différents mondes souhaités. Le rapport entre ces derniers peut s'articuler de plusieurs façons : comme un rapport d'adéquation (les deux agents désirent le même monde possible), un rapport de compétition (les deux mondes souhaités s'excluent mutuellement) ou encore un rapport d'indifférence (les deux mondes ne s'excluent aucunement), avec différentes positions intermédiaires. ${ }^{154}$ Il faut aussi noter que chaque personnage, toujours, agit selon son monde perçu, et n'entre pas en conflit avec le monde souhaité d'un autre, mais plutôt avec ce qu'il croit être son monde souhaité. La situation dramatique présentée par Gervais comme le conflit entre deux schèmes

\footnotetext{
${ }^{153}$ Mentionné dans notre deuxième chapitre, il s'agit d'une rencontre conflictuelle entre deux forces dans la pensée de Gervais.

154 Si nous développons notre terminologie, la base est tirée de Marie-Laure Ryan - Possible Worlds, Artificial Intelligence, and Narrative Theory, p.122-123.
} 
interactifs occupe ainsi, pour Ryan, une position intéressante, puisqu'elle place les différents personnages en relation, alternant interprétation et action, comme le modèle de la lecture d'Aarseth le fait avec l'usager et le cybertexte.

Nous revenons ainsi sur le travail de lecture cybertextuelle et par extension, sur la lecture vidéoludique. L'intérêt du jeu électronique comme œuvre de fiction, nous l'avons mentionné maintes fois, réside pour nous dans le rapport entre ses règles fictionnelles et ludiques, entre son récit et son jeu. Au plan de la lecture, nous suivons la même lancée, aussi proposons-nous de considérer l'agent-joueur comme tenant, essentiellement, le rôle du personnage dans l'approche de Ryan. Le jeu électronique, en effet, place le joueur à la fois dans le rôle d'un lecteur, mais aussi celui d'un agent, et les deux réalités sont fortement liées. Si la logique systémique de Ryan permet de mieux comprendre la façon dont les personnages sont construits en analysant ses actions, elle peut alors nous permettre de mieux cerner ce qui est demandé et imposer au joueur en tant qu'agent.

L'action du joueur, ainsi, est guidée par sa lecture interprétative du jeu - la synthèse de ce qui est offert par le moteur de représentation du jeu. Cette synthèse est en soi un travail de lecture, qui pousse le joueur à constituer son réseau de mondes possibles privés, avec en premier lieu un monde perçu. Tout comme pour un personnage, il ne s'agit que d'une perception, construite d'inférences (rentables), qui peut donc être erronée, voire trompée intentionnellement. De là, le travail de lecture du joueur consiste à établir les différents mondes accessibles, et à leur attribuer différentes valeurs de probabilité, de désirabilité et de 
moralité afin de déterminer son monde anticipé et son monde souhaité. Se basant sur ces constructions, le joueur peut alors entrer en communication, agir sur le programme, sélectionnant les actions pour atteindre son monde souhaité. Celles-ci sont ensuite analysées et enclenchent les procédures adéquates pour permettre au jeu électronique de modifier sa représentation, voire son monde textuel actuel en prenant en considération les actes du joueur et de son personnage.

La lecture vidéoludique se déroule ainsi comme une communication constante entre programme et joueur, ce qui crée à la fois l'expérience de la fiction, et l'expérience de jeu. La lecture interprétative, nous la voyons comme la construction de mondes possibles, et les autres aspects de la lecture, présentés par Aarseth (exploratoire, configurative et textonique) sont des constructions de l'œuvre, face aux actions de son usager. Ces dernières, en plus d'obéir à la logique de l'action dont nous venons de discuter, ne sont pas totalement libres, et obéissent à certaines contraintes. L'usager du cybertexte, dans notre cas le joueur, ne peut par exemple communiquer avec l'œuvre qu'à l'aide des méthodes prévues à cet effet.

C'est pourquoi, lors de notre analyse, nous nous concentrerons sur les contraintes imposées par chaque jeu à la lecture vidéoludique. Spécifiquement, nous chercherons à voir comment chaque jeu encadre la lecture et les actions possibles du joueur. Nous commencerons donc par étudier de quelles façons est représenté le monde textuel actuel $\mathrm{du}$ jeu. Surtout, nous nous attarderons sur comment les perceptions du joueur sont 
manipulées par le programme, de façon à rendre plus rentables certains mondes perçus, anticipés ou souhaités, et de façon à diriger 1'évaluation des dénouements possibles. Plus encore, nous examinerons quelles voies de communication sont employées, et donc quelles contraintes sont imposées aux efforts d'exploration de configuration ou de textonique du joueur. Finalement, nous essaierons de voir quand et comment le joueur est placé en relation avec d'autres agents, pour voir comment s'articulent les situations dramatiques de lecture.

\section{Fallout 3}

Fallout 3 est un représentant d'un genre souvent célébré pour la grande liberté accordée au joueur, celui du «open world», littéralement «monde ouvert». Nous avons vu précédemment comment ses systèmes de règles et de propriétés accordent une grande place aux contributions du joueur, il nous reste maintenant à voir comment ce jeu gère le travail de lecture présenté en début de chapitre.

La façon dont est représenté l'univers de Fallout 3 nous montre, dès le premier abord, que le jeu met l'accent sur l'exploration et l'interprétation du joueur. Le jeu présente initialement au joueur un monde serein, contrôlé et futuriste - avant de le catapulter dans un monde extérieur dont il ne connaît rien. Autrement dit, une fois les premiers moments du jeu passés, le joueur est placé dans la même position que son personnage, ignorant tout (ou presque) du monde fictionnel auquel il est confronté. La construction du monde perçu, et des mondes possibles dérivés, s'opère ainsi à l'aide d'informations fragmentaires, 
minimales, et demande au joueur d'agir bien souvent sans comprendre toutes les implications de ses gestes. Ce faisant, Fallout 3, nous pourrons le constater, demande au joueur d'inférer un grand nombre d'éléments et de confronter sa vision du monde (à travers ses actions) au monde actuel.

Explorons d'abord les modes de communication entre joueur et jeu, à commencer par le fonctionnement des dialogues et des conversations. À chaque échange, le joueur de Fallout 3 est amené non seulement à interpréter réactions et réponses de son personnage (ce qui est en soi un travail de lecture), mais (surtout) à sélectionner celles qui lui semblent les plus appropriées. Il s'agit de l'exemple le plus simple et efficace des voies de communication tout au long de l'expérience de jeu : le programme présente une situation (conversation) face à laquelle le joueur doit imaginer différents mondes possibles (répliques) et, au final, en sélectionner un qui sera pris en compte par le programme, poursuivant ainsi l'échange. La liberté d'imagination et de sélection n'est pas complète, cependant. Chaque réplique doit être sélectionnée parmi plusieurs choix proposés par le jeu, dont quelques-uns ne sont offerts que si certaines caractéristiques du personnage sont suffisamment élevées - ce qui revient à dire que les possibilités (du moins celles qui peuvent être prises en compte par le programme) sont dictées par les règles du jeu et de la fiction. On peut voir ce fonctionnement sur cette capture d'écran : 


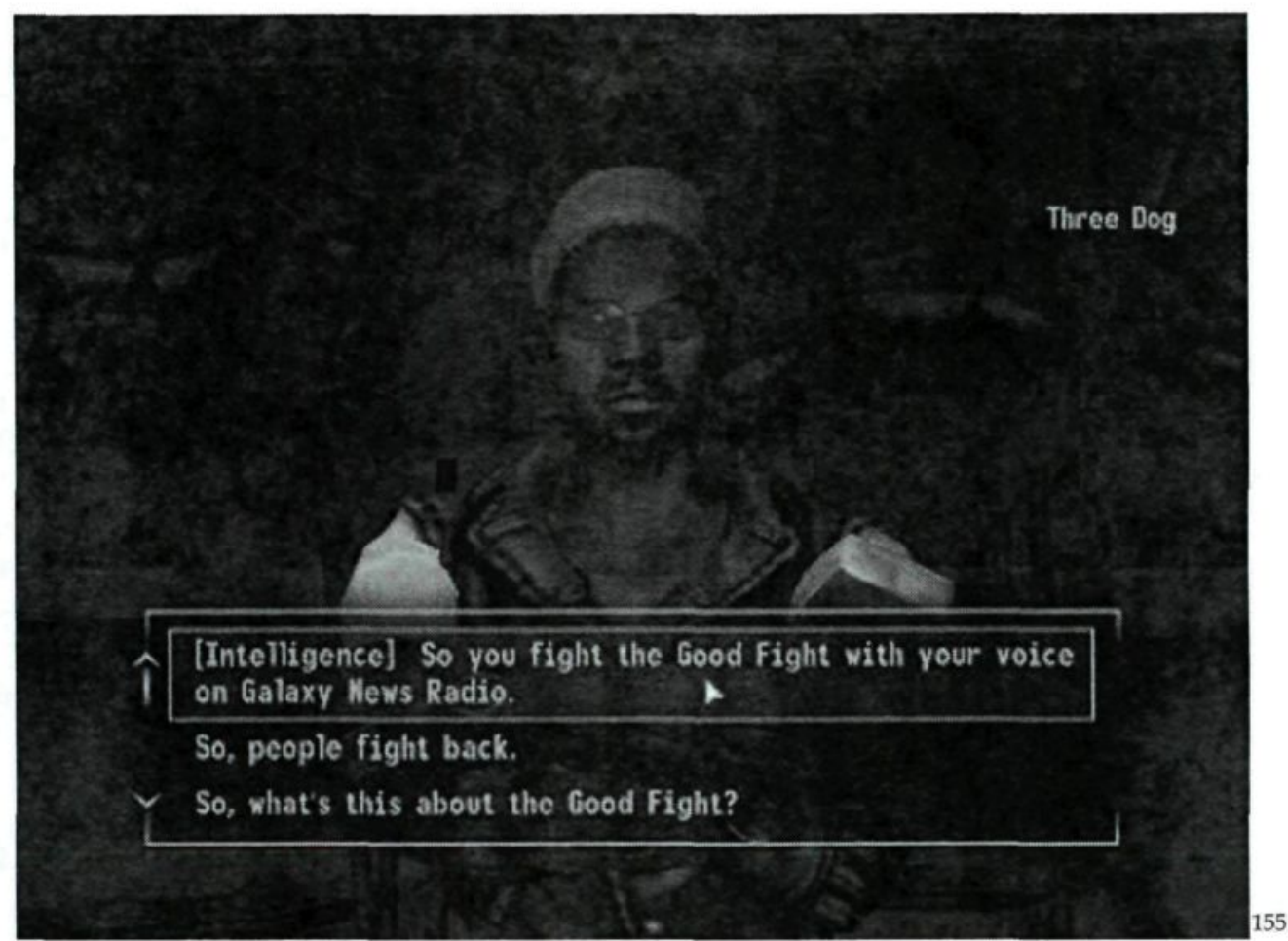

Il est à noter ici que la première option est affublée d'une mention entre accolades («Intelligence »), ce qui signifie que cette possibilité n'est offerte qu'à un personnage dont la statistique d'intelligence est suffisamment élevée. Autrement dit, il s'agit d'un monde possible qui ne peut être pris en compte par le programme que si les règles ludiques et fictionnelles le permettent: il n'est accessible qu'à un joueur dont le personnage présente certaines caractéristiques.

Ainsi, le système de dialogue, en apparence, permet une certaine variété de configuration en laissant le joueur choisir lequel des mondes possibles proposés sera actualisé. Chaque possibilité implique également certaines conséquences qui ne sont pas toujours apparentes, accordant une part d'incertitude au choix des possibles, et c'est là que se situe

155 Bethesda Game Studios - Fallout 3. (Galaxy News Radio - Galaxy News Radio) 
$l^{\prime}$ une des grandes forces du jeu. Autrement dit, il est tout à fait envisageable que les actions exécutées par le joueur ne mènent pas à son monde souhaité, ou du moins, pas entièrement, puisque le joueur détermine ses actions selon son monde perçu, recomposé à partir de la représentation fragmentaire du monde textuel actuel. Pour mieux saisir cet aspect, prenons l'exemple d'une quête à branchements multiples qui se résout en majeure partie par le dialogue : «The Power of the Atom ».

- La quête concerne une bombe atomique encore intacte située en plein cœur d'un village de réfugiés appelé Megaton. Deux personnages s'y intéressent plus particulièrement: le shérif du village, qui cherche quelqu'un pour la désarmer, et l'énigmatique M. Burke, qui offre une récompense substantielle à celui qui pourra provoquer son explosion. Outre les options de dialogue évidentes (accepter ou refuser l'offre, ou encore négocier une meilleure récompense), il existe quelques nuances à la quête. Un joueur ayant eu l'occasion de discuter avec M. Burke peut, notamment, retourner voir le shérif pour dénoncer le malfrat. C'est là qu'intervient l'incertitude mentionnée plus tôt: la dénonciation mène à une tentative (plutôt prévisible) d'arrestation. Par contre, une fois accusé, M. Burke dégaine un pistolet, assassine le shérif, et fuit la scène. La possibilité choisie par le joueur est ainsi porteuse de conséquences qui peuvent être imprévues, parfois désirables, ou, comme dans le cas présent, peu souhaitables.

L'essentiel, donc, est que l'usager, bien qu'il puisse influer sur le cours des dialogues et des actions, doit inférer non seulement le développement possible du monde textuel, mais 
aussi les «dommages collatéraux » du développement. Le monde fictionnel de Fallout 3 est glauque et cynique, et il est fort probable qu'un joueur qui désire y accomplir certaines actions teintées d'idéalisme doive confronter sa vision à celle du jeu. C'est, somme toute, là que se situe l'une de ses grandes richesses. En laissant le joueur subir les conséquences de choix en disharmonie avec l'univers fictionnel, Fallout 3 utilise les mécanismes de lecture comme levier narratif. Ainsi, la mort du shérif dans « The Power of the Atom » exemplifie la dureté du monde fictionnel : un personnage qui s'imagine régler ce genre de malfaisance par un simple appel à l'autorité n'a d'autre choix que de constater l'incompatibilité de sa vision, de ses principes moraux en quelque sorte, avec celle du jeu. Plutôt que de ne jamais actualiser cette possibilité, voire de ne pas l'offrir, le programme intègre les choix du joueur à sa fiction, puis corrompt et subvertit son monde souhaité.

Ces processus de confrontation entre les mondes souhaités par le joueur et les mondes projetés en réponse par le jeu sont essentiels dans l'expérience de lecture de Fallout 3 . Le défi, pour le joueur, est de bien comprendre les « règles » internes de l'univers de la fiction, pour mieux pouvoir construire un monde anticipé qui y est probable - et pour savoir à quoi s'attendre de chaque action. Le jeu maintient une cohérence certaine en contestant certains éléments des actions du joueur qui présentent de trop grands glissements par rapport au monde actuel de la fiction. Ainsi, subtilement, lire un jeu comme Fallout 3 devient nécessairement un exercice complexe d'interprétation pour déterminer dans quelles directions le monde textuel actuel peut se développer. Plus qu'un travail de lecture, il s'agit d'un véritable jeu de rôles, où le joueur est amené à penser 
comme un agent interne de la fiction, plutôt que comme un stratège externe $-l^{\prime}$ ambition étant de devenir une réelle « simulation » au sens où l'entendait Frasca.156

D'un point de vue ludique, Fallout 3 présente aussi un certain degré de communication. Un joueur doit savoir anticiper le comportement d'ennemis, par exemple, pour choisir la tactique de combat qui conviendra le mieux - lui faire face, s'enfuir, utiliser une arme différente, etc. Si ces choix peuvent être narrativisés, à plus forte raison considérant l'importante intégration entre jeu et fiction, ils n'en demeurent pas moins des choix ludiques, issus d'une interprétation des règles de jeu. On assiste ainsi, en combat, à un rapide échange, qui est clairement un rapport de conflit: l'intelligence artificielle qui commande les adversaires s'adapte à la situation pour éliminer le personnage, et le joueur fait de même face à son adversaire, leurs mondes souhaités étant incompatibles.

Finalement, nous souhaitons nous pencher sur les mécanismes de rentabilité offerts par le jeu. Nous avons vu d'abord les fréquentes confrontations entre les mondes possibles construits par le joueur (surtout son monde perçu et son monde souhaité) et le monde textuel actuel. C'est déjà là une forme de rentabilité suggérée : en opérant ainsi, Fallout 3 rend certains développements plus accessibles que d'autres, et, à force de construire de façon plus efficiente son monde perçu, le joueur peut mieux rentabiliser ses actions pour atteindre son monde souhaité. D'un point de vue ludique, également, le jeu rentabilise différents ensembles d'actions. Par exemple, certaines actions sont affublées d'un gain ou

${ }_{156}$ Frasca aborde le jeu électronique comme une simulation de conditions possibles, où le joueur doit prendre la place de son personnage, et simuler une action logique dans notamment Videogames of the Oppressed Videogames as a Means for Critical Thinking and Debat. 
d'une perte en « karma », qui mesure la qualité morale du personnage : voler fait baisser le karma, sauver la vie d'un marchand le fait monter. En soi, la mesure semble innocente, mais on constate assez tôt qu'un personnage au karma bas s'attire des ennuis : personne ne veut traiter avec lui, par exemple. $S^{\prime}$ il sombre extrêmement bas, des chasseurs de prime se mettent alors à attaquer le personnage. C'est là une méthode de rentabilité : en punissant les actes répréhensibles moralement (selon le monde textuel), Fallout 3 affirme ses conventions morales, rendant plus rentable, notamment, une certaine interprétation de l'axe de moralité du monde.

Un autre exemple peut être vu dans la récompense en expérience : face à une situation de jeu, différentes actions peuvent être récompensées de différentes façons, rendant plus « rentable » une configuration offrant une meilleure récompense. Ainsi, comme nous l'avons déjà mentionné, le jeu permet différentes interprétations et différentes actions, mais, en récompensant certaines plus que d'autres, il suggère une lecture spécifique de son monde fictionnel. Il est également intéressant de noter que l'emploi de récompenses intangibles (l'expérience, après tout, est un élément purement ludique) peut en venir à rentabiliser certains mondes fictionnels à l'aide d'incitatifs ludiques - ce qui nous ramène à l'intégration entre jeu et fiction, décrite dans notre premier chapitre.

La lecture de Fallout 3 est ainsi hautement axée sur l'interprétation et l'exploration: le joueur doit explorer pour mieux interpréter le monde et rentabiliser ses actions. Toutefois, l'œuvre laisse aussi une grande latitude quant à la lecture configurative, et s'ajuste à de 
nombreuses actions possibles. Surtout, en fait, il utilise la liberté de configuration du joueur pour créer différents effets, en laissant le joueur assumer les éléments indésirables entraînés par ses actions. Les différentes configurations de l'œuvre sont ainsi basées sur un monde fictionnel qui n'est pas influencé par le joueur, mais qui se laisse plutôt explorer, parcourir aux risques et périls du personnage.

\section{World of Warcraft : Wrath of the Lich King}

Les jeux en ligne comme World of Warcraft présentent un cas de figure fascinant en ce qui concerne la place accordée à l'usager et à son travail de lecture dans leur progression narrative. En mettant l'accent sur leur aspect massivement multijoueurs, ils placent le joueur à la fois dans le rôle du personnage principal de leur fiction personnelle, et dans celui d'un personnage mineur du grand ensemble qu'est leur monde fictionnel. Nous pourrons voir dans le cas de WoW que cette dualité du joueur-agent comporte son lot d'implications en ce qui concerne la lecture vidéoludique. Dans le même ordre d'idée, nous nous attarderons à l'interaction entre joueurs comme phénomène de lecture particulièrement présent dans ce jeu.

Avant de passer à l'étude de l'influence de l'aspect massivement mutijoueurs de WoW sur la lecture, toutefois, nous désirons nous pencher en premier lieu sur les mécanismes d'interaction fictionnelle (par le biais de dialogues et conversations) entre le programme et le joueur. Surtout, nous voulons montrer qu'il s'agit là d'un jeu qui encadre de façon très

minimale sa fiction (ce que nous avons déjà effleuré dans notre premier chapitre), 
capitalisant sur l'imagination du joueur, tout en encadrant énormément son côté ludique, et avec celui-ci, les actions du joueur. Du point de vue de la lecture, cela signifie que WoW emploie de nombreux mécanismes de rentabilité, que nous examinerons par la suite.

D'emblée, sur le plan des interactions discursives entre le joueur et le programme, on peut repérer l'approche minimaliste du jeu face à la fiction. Sitôt sorti de l'écran de création de personnage, on peut voir quelque chose comme ce qui suit :

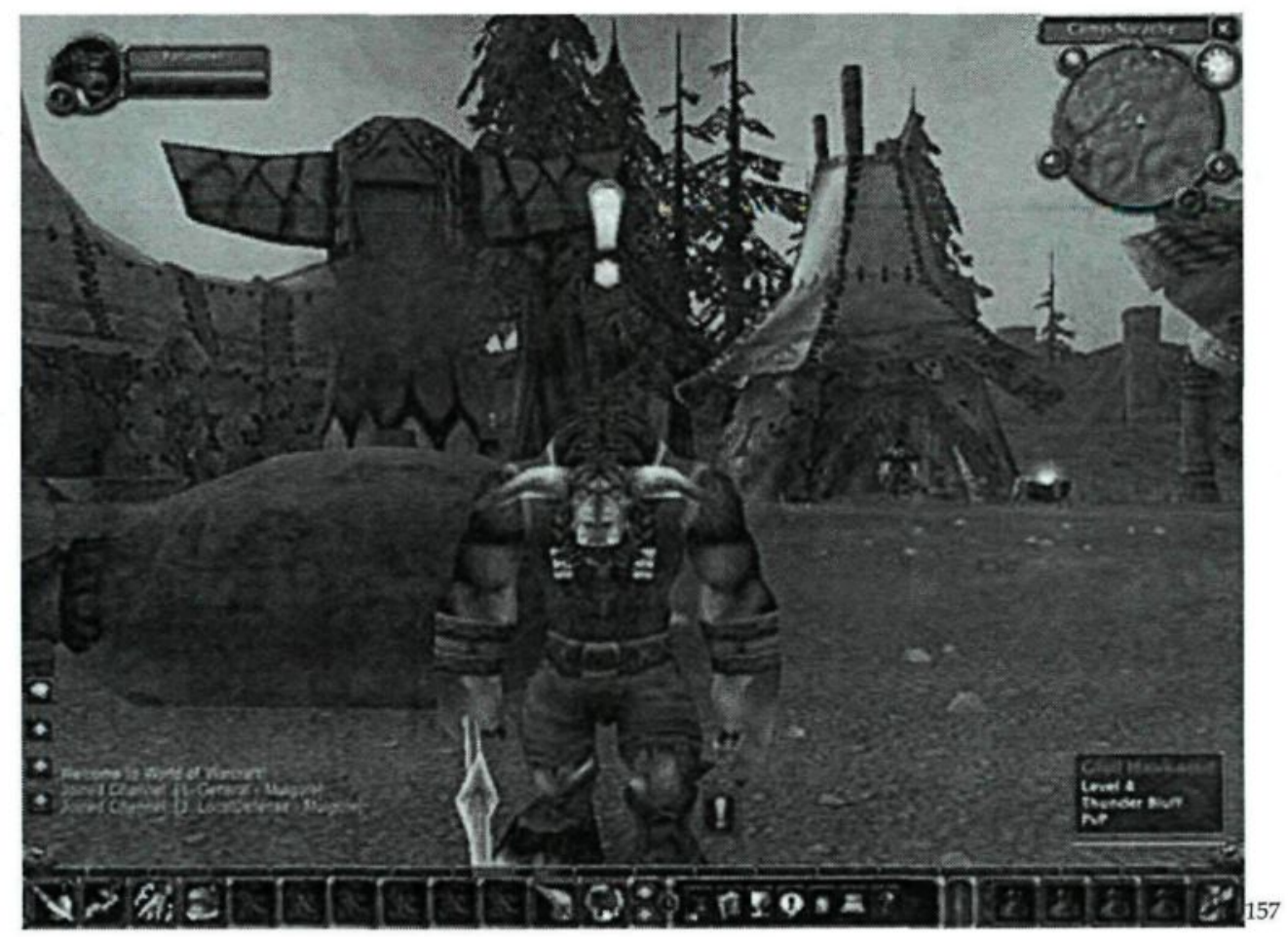

Adornant le personnage non-joueur présent sur l'image se trouve un point d'exclamation (signe ludique difficile à lire comme élément fictionnel) qui indique qu'il s'agit là d'un personnage avec qui il est possible d'interagir - en l'occurrence, afin d'ouvrir une conversation qui mène à une quête. S'il est possible d'entrer en contact avec d'autres

${ }^{157}$ Blizzard Entertainment - World of Warcraft: Wrath of the Lich King. (Mulgore) 
personnages, il n'est pas rentable de le faire : ceux-là n'auront rien à offrir au joueur, que ce soit d'un point de vue ludique ou fictionnel.

Contrairement à Fallout 3, World of Warcraft ne présente alors pas le dialogue comme un échange entre le personnage joueur et un personnage non joueur : il s'agit plutôt d'une transmission simple d'informations, le joueur en étant le récepteur. Son avatar est ainsi un protagoniste silencieux, et s'il est permis au joueur de s'imaginer ses réactions et échanges, le jeu fait l'économie de la planification de différents scénarios. Si le joueur ne peut ainsi échanger réellement avec le programme, il lui est néanmoins laissé une plus grande latitude dans l'imagination des réactions de son personnage, qui n'est pas contraint de répondre d'une façon prédéterminée par le jeu. Sa lecture interprétative de l'échange n'est ainsi que très peu balisée par le programme.

On peut observer le même phénomène d'encadrement minimaliste à propos de la fictionnalisation des règles du jeu. S'il est possible d'attribuer un sens fictionnel à de nombreux procédés ludiques (le gain d'expérience, l'apprentissage de nouvelles compétences ou les récompenses monétaires des quêtes, par exemple), World of Warcraft ne propose qu'incidemment certains mondes possibles. Cette façon de fonctionner est cohérente avec la ségrégation observée entre règles ludiques et règles fictionnelles - le programme se préoccupe de fournir les premières et laisse le travail de lecture entre les mains du joueur. Un minimum d'éléments est décrit par le jeu, laissant libre cours aux interprétations pour deviner les motivations des personnages, leur histoire, leur attitude, 
et de nombreux autres éléments. Malgré son apparente complexité, World of Warcraft demeure très «traditionnel » et similaire aux médias sans support informatique à cet égard.

Les interactions limitées entre le joueur et le programme proposent généralement au joueur différentes quêtes, dont nous avons discuté amplement dans notre deuxième chapitre. Si le joueur peut explorer librement le monde de WoW, les signes et balises mises en place par le programme rendent rentables des enchaînements très précis : les points d'exclamations (comme dans notre première image) incitent le joueur à interagir avec les personnages offrant des quêtes, ces dernières offrent alors des récompenses rendant leur accomplissement des plus désirables tant du point de vue ludique que fictionnel, et une fois qu'elles sont acceptées, leurs objectifs apparaissent dans l'interface, encourageant le joueur à diriger son personnage dans des zones précises. WoW encadre ainsi l'expérience de jeu en rendant rentable une lecture très précise de ses éléments : il offre une grande liberté d'actions, mais s'assure d'affirmer la supériorité de certaines en employant différents mécanismes de rentabilité.

Ces derniers se situent alors essentiellement du côté des règles de jeu : des gains en ressourcés ou en expérience, par exemple, encouragent certains mondes anticipés ou souhaités. Il est une catégorie de règles, cependant, qui nous semble particulièrement pertinente, puisqu' elle est un incitatif à la fois ludique et fictionnel : celles concernant la réputation. La réputation, dans World of Warcraft, est une mesure numérique de 
l'appréciation qu'ont différentes factions (groupes, clans, villes, sociétés, etc.) des actions du personnage joueur. L'intérêt de la réputation est qu'il s'agit d'abord de règles de jeu, mais que leur présence contribue à consolider, pour le joueur, l'axe de moralité essentiel à son processus de prise de décision: l'interprétation de ces règles constitue ainsi un phénomène de lecture particulièrement éloquent. Pour illustrer son fonctionnement, nous allons nous pencher sur deux factions opposées : les gobelins de "Booty Bay», et les pirates du clan des « Bloodsail Buccaneers. »

La réputation fonctionne sur une échelle, allant de la plus négative (« détesté ») à la plus positive («exalté »). Entre les deux, on retrouve divers degrés, dont la progression va comme suit: hostile, inamical, neutre, amical, honoré, révéré. En ce qui concerne les factions choisies, le point de départ face à Booty Bay est le rang « neutre », tandis que celui face aux Bloodsail Buccaneers est « hostile. » En termes de jeu, cela veut dire qu'un joueur n'ayant jamais eu à faire avec l'une ou l'autre des deux factions sera attaqué à vue par les pirates, mais pourra commercer avec les gobelins.

La façon dont le joueur peut augmenter sa réputation avec ces deux factions est d'une simplicité désarmante: les deux se détestent, et ainsi l'élimination de personnages appartenant à l'une rapporte au joueur des points avec l'autre. Sans grande surprise, le meurtre gratuit de membres d'une communauté est plutôt mal perçu par ses semblables, et nuit fortement à la réputation associée. Les pénalités sont cependant bien plus grandes : on parle d'un gain d'environ 5 points d'un côté, pour une perte d'environ 75 de l'autre. 
De manière à rendre l'association avec un clan ou l'autre plus rentable, différents niveaux de réputation rendent accessibles diverses récompenses: le droit, d'abord, de ne plus se faire attaquer à vue, mais aussi des pièces d'équipement rares, ou des rabais chez les marchands associés.

Il n'y a donc pas dans World of Warcraft de mesure globale du «karma » ou de la « gentillesse » du personnage joueur. Dans l'exemple que nous avons mentionné, une même action (le meurtre d'un garde) est perçue comme désirable par une faction, et indésirable par l'autre. Certes, le jeu encourage l'association à l'une d'elles ${ }^{158}$ mais il permet l'association contraire. Le code moral qui s'en dégage est donc plus relatif que celui de Fallout 3, et la valeur de moralité attribuée aux différentes actions du joueur s'y ajuste nécessairement. C'est donc dire qu'à travers son système de jeu, une représentation numérique de la valeur des actions du joueur, World of Warcraft parvient à représenter une vision du monde, que le joueur doit intégrer - ce qui est un phénomène de lecture particulièrement insidieux.

Les relations conflictuelles entre le joueur et d'autres éléments du jeu, que nous retrouvons surtout en situation de combat, sont un autre exemple intéressant des mécanismes de rentabilité mis en place par le programme. Les situations de combat sont des échanges assez complexes, où en apparence plusieurs mondes possibles s'offrent au joueur pour

\footnotetext{
158 Booty Bay, après tout, ne harcèle pas le joueur de prime abord, et contrôle une bonne partie de la région, aussi est-il rarement justifiable de s'opposer à eux. Plus encore, sa réputation est liée à celle de trois autres clans gobelins répandus un peu partout dans le monde, accentuant ainsi les pénalités pouvant être associées à une mauvaise entente entre le jouteur et la faction.
} 
modeler son action. Contrairement à l'intelligence artificielle de Fallout 3, cependant, celle de World of Warcraft offre très peu de capacités d'adaptation : les actions du joueur sont minimalement prises en considération par le jeu, qui ne reconfigure que très rarement ses actions propres après les avoir analysées. Le programme se contente généralement d'exécuter une série d'actions prédéfinie, explicitant et actualisant un monde anticipé unique, cohérent et prévisible. Une situation de combat devient ainsi presque une séquence programmée, où le joueur doit exécuter le plan nécessaire pour réussir, et poursuivre son expérience de jeu. En gros, le jeu ne rend accessible qu'un faible nombre de mondes possibles, imposant des développements d'actions hautement normalisées : ce faisant, il réduit les possibles que le joueur doit évaluer pour déterminer ses actions, et n'en rend rentable qu'une poignée aisément identifiables.

On observe ce phénomène plus particulièrement dans les combats de groupe contre des ennemis puissants. Ces combats se présentent plutôt sous la forme de puzzles que sous la forme d'une interaction réelle, exigeant de la part des joueurs l'exécution de stratégies bien spécifiques. ${ }^{159} S^{\prime}$ il peut exister plusieurs stratégies pour contourner certaines difficultés, les joueurs doivent en général actualiser des séquences précises sous peine d'échouer (avec pour seule conséquence de devoir recommencer). Certains rôles demandent une plus grande réactivité, mais la grande prévisibilité des combats et leur répétition ${ }^{160}$ installent

\footnotetext{
159 Comme se positionner à certains endroits précis, se déplacer à intervalles réguliers et chronométrés, éliminer d'autres ennemis mineurs qui se joignent au combat, etc.

160 Un ennemi vaincu ne l'est pas toujours définitivement et il est fort probable que le joueur ait à participer au même combat à plusieurs reprises.
} 
une logique d'actualisation: au moment $X$, il faut accomplir $Y$, et la progression se poursuit.

Les combats entre joueurs n'obéissent évidemment pas du tout à la même logique et, considérant leur plus grande part d'imprévisibilité, se déroulent beaucoup plus à la manière d'une réelle relation d'échange, de lecture interprétative et d'actions adaptées au contexte. Les plans de plusieurs joueurs s'opposent, et ne sont souvent inférés que partiellement, aussi faut-il pour chacun adapter son plan aussi rapidement que possible. Cette particularité nous amène à notre second point, concernant l'accent mis par WoW sur son aspect massivement multijoueurs, encourageant entre les joueurs des interactions complexes, exigeant ainsi un travail de lecture bien plus raffiné. Si certaines interactions entre joueurs peuvent être comprises comme extérieures au jeu (deux amis discutant de leur fin de semaine), beaucoup sont d'abord ludiques (tel les situations de combat mentionnées précédemment), et peuvent aisément être lues comme faisant partie de la fiction. Ces échanges entre joueurs peuvent alors se comprendre comme des situations dramatiques, se plaçant au cœur des actions de chacun.

Ainsi, cinq joueurs prenant d'assaut, en groupe, un donjon sont en train de travailler ensemble pour atteindre le même monde souhaité - ou du moins, différents mondes souhaités qui ne sont pas incompatibles. Quatre autres s'affrontant dans l'arène poursuivent des mondes souhaités complètement incompatibles, et se situent en compétition. Dans les deux cas, ces interactions sont non seulement encouragées par le 
jeu, mais même nécessaires à la progression des personnages, que nous avons identifiée au deuxième chapitre comme l'objectif final de la progression de World of Warcraft. Les situations dramatiques offertes par l'interaction entre joueurs ne sont ainsi pas seulement des possibilités, mais bien des impératifs de son expérience de lecture.

Un des aspects les plus importants de cette interaction entre joueurs est la capacité de communiquer par texte à l'intérieur même du jeu. Les discussions entre joueurs sont alors prises en charge par le système - le joueur tape ce qu'il désire voir son personnage chuchoter, dire ou crier, et le système l'affiche, tout bonnement. Il s'agit là d'un exemple restreint de travail textonique : le joueur peut employer les termes, mots et symboles qui lui plaisent, et les voir intégrés à la fiction. Certes, les seuls éléments du jeu qui peuvent réellement analyser, comprendre et s'adapter à ces fragments textuels sont les autres joueurs : c'est pourquoi nous parlons d'un exemple plutôt restreint. Qui plus est, le jeu lui-même n'encourage pas particulièrement l'emploi de conversations fictionnalisées, aussi la volonté de se présenter comme un élément de la fiction et d'être perçu comme tel par les autres joueurs relève-t-elle entièrement d'une logique et d'un désir personnels, n'étant encadrée par aucun mécanisme de rentabilité par le jeu.

Cela étant dit, les différentes actions entreprises dans le cadre de ces interactions, même en dehors des conversations, ne se voient que très rarement attribuer un sens fictionnel. Aussi World of Warcraft peut-il se contenter de laisser les joueurs gérer entre eux le niveau de fictionnalité associé à chacun de leurs gestes. La compréhension fictionnelle n'y est pas 
nécessaire, et il est fort possible de ne «lire » le jeu que comme un ensemble de règles ludiques, aussi toute «lecture » fictionnelle repose-t-elle essentiellement sur un travail d'interprétation, minimalement encadré par le jeu. Un joueur qui vend du minerai à un autre peut ne voir sa transaction que comme un échange de données, mais il est surtout à même de l'interpréter comme du commerce à l'intérieur de la fiction, ainsi que la représentation de cette action par World of Warcraft 1'indique.

Hormis les interactions entre joueurs, la tendance à régler au quart de tour les actions nécessaires au plan ludique et à utiliser le travail de lecture fictionnelle de façon très traditionnelle est à l'évidence un choix conscient de la part des développeurs de $W o W$, considérant qu'elle va s'alourdissant. Les derniers développements ajoutent au jeu un « journal de donjon » qui vient expliquer en détail le fonctionnement des combats les plus complexes, et donner une bonne quantité d'informations fictionnelles sur les ennemis à abattre. Cela revient, donc, à dire que l'interprétation fictionnelle est officiellement reléguée à un statut optionnel : libre au joueur d'inférer (en respectant le cadre des règles de la fiction) ce qu'il veut, World of Warcraft ne cherche pas à utiliser le travail de lecture fictionnelle pour créer des effets quelconques. Le fort encadrement des combats et de la progression ludique donne un jeu efficace, facile d'approche, mais qui conserve un contrôle énorme sur les actions possibles du joueur.

Autrement dit, avec un monde textuel très vaste, et très peu de possibilités de reconfiguration permanente de ses éléments, World of Warcraft laisse le joueur être un 
lecteur plutôt interprétatif, mais surtout exploratoire. Si le texte lui-même ne varie pas (puisque minimaliste), il peut être lu et relu, et parcouru dans un ordre différent à chaque fois - dans la perspective $d^{\prime}$ Aarseth, cela voudrait dire que cette œuvre est plus près de l'hypertexte (lecture interprétative et exploratoire seulement, monde statique) que du cybertexte. Toutefois, nous avons aussi vu que certains éléments sociaux du jeu se rapprochent $d^{\prime}$ un travail textonique, et placent les joueurs dans une dynamique de conflit externe. C'est là que se situe la grande force de $W o W$, du moins au niveau de la lecture: en étant basé sur des interactions complexes, qui prennent alternativement la forme de compétition ou de coopération, ce jeu pallie sa relative simplicité narrative, nécessaire pour maintenir la cohérence fictionnelle. Enfin, si la fiction doit être plutôt imaginée, les règles de jeu créent tout de même nombre d'effets de lecture - le code moral souple qui se dégage du système de réputation, dont nous avons discuté, en est un exemple.

\section{Valkyria Chronicles}

De par son fonctionnement très proche des médias traditionnels, il est naturel que Valkyria Chronicles accorde un statut similaire au rôle du joueur face à sa fiction. En effet, si nous défendons l'idée du joueur traité comme personnage, il nous faut admettre ici que plus souvent qu'autrement, il demeure spectateur - ce qui ne veut pas dire que son travail de lecture n'est pas nécessaire. Par contre, que Valkyria Chronicles appartienne à la grande famille des jeux de stratégie l'amène à accorder, comme nous pourrons le constater, une importance capitale au joueur et à ses stratégies d'un point de vue ludique, et le replace au centre de l'action. 
Concernant la place du joueur face à la fiction, et son rôle en tant que lecteur, nous proposons ici aussi comme point de départ l'analyse des dialogues et des choix de conversation, nous permettant de mieux cerner les voies de communication offertes par le jeu. À cet égard, Valkyria Chronicle se fait très clair : le joueur n'a, à aucun moment, de réelles possibilités de conversations. Certains dialogues sont représentés, toutefois, mais ils sont intégrés à des scènes cinématographiques, qui ne sont pas influencés par le joueur. Il demeure certes possible d'y effectuer une lecture interprétative, mais toute construction de mondes possibles demeure, pour le joueur, non communiquée, du moins dans le cadre de la conversation - interdisant, du coup, toute lecture dynamique. Alors que WoW crée plutôt une illusion participative, ce jeu-ci présente clairement son monde fictionnel comme un film animé, duquel le joueur est spectateur.

Les dialogues et conversations qui permettent au joueur de Fallout 3 d'interagir avec le programme et de définir son personnage à travers ses choix et actions sont donc utilisés par Valkyria Chronicles de façon plus conventionnelle. Ils permettent au joueur de saisir les personnages impliqués (y compris le personnage principal, sur lequel le joueur n'a aucune emprise), à travers son interprétation de leurs actions, commentaires et interventions : c'est donc sa lecture interprétative qui est sollicitée, à l'exclusion de toutes les autres. S'il existe bel et bien des voies de communication permettant au joueur de tenter d'actualiser son monde souhaité (et non celui de personnages extérieurs), elles sont essentiellement consacrées à l'aspect ludique. 
Nous identifions deux voies de communication principales dans Valkyria Chronicles, toutes deux liées au côté stratégique du jeu. La première se trouve dans le camp d'entraînement, à un niveau préparatoire, et la seconde en situation de combat. Dans les deux cas, l'interaction est foncièrement ludique, mais peut être interprétée, à force d'imagination, comme faisant partie de la fiction. Il demeure qu'elle implique une certaine lecture dynamique de la part du joueur, que nous pourrons examiner.

Au camp d'entraînement, par exemple, il existe une certain forme de conversation - un interface rudimentaire permet de contacter des personnages (entraîneur, mécanicien, recruteur) qui peuvent développer certains aspects des forces militaires contrôlées par le joueur en échange de diverses ressources (argent, expérience) accumulées en cours de partie. Tout cela se fait de façon très mécanique, avec un minimum de représentation :

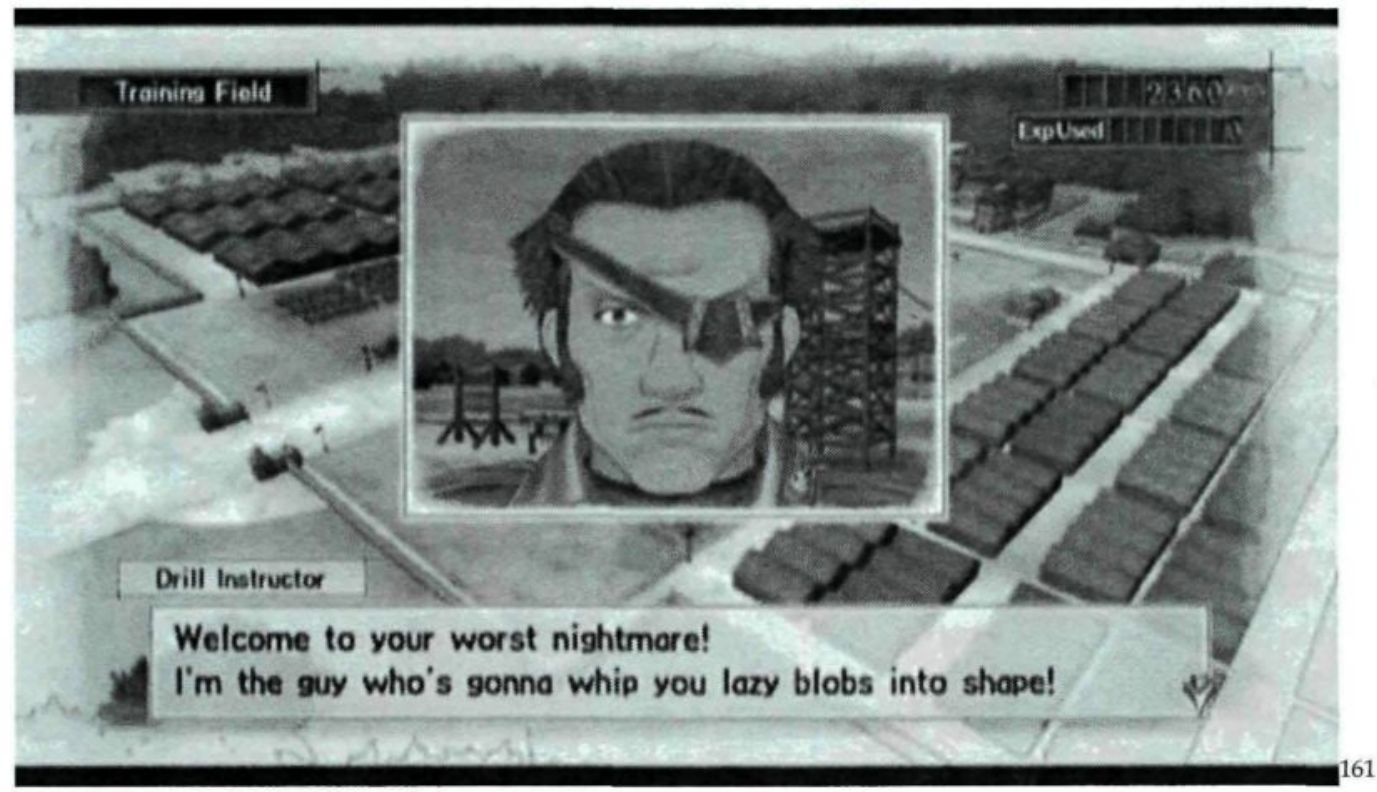

${ }^{161}$ Sega - Valkyria Chronicles. (Training Field) 
Sur l'image ici présentée, on peut voir l'inventaire des ressources (en haut à droite) et la représentation de l'instructeur, qui, apparemment, s'adresse aux troupes. C'est là toute la fictionnalisation présente - l'entraînement n'est pas représenté, ni même le lieu, au-delà de l'arrière-plan. Ainsi, l'essentiel de la lecture, ici, est fondée sur l'imagination et les projections du joueur, avec peu d'indices lui permettant de développer un monde perçu rentable. Outre les scènes animées, ce type de présentation est hautement présent, et capitalise donc sur un travail de lecture pour s'exprimer, optant pour une représentation minimaliste. Cette approche rappelle World of Warcraft, se concentrant sur l'aspect ludique, et laissant le joueur faire à sa mesure, à travers sa lecture, sa fiction. La différence principale réside justement dans la présence des éléments cinématographiques, qui viennent donner un fil conducteur narratif, et qui sont majoritairement absents de WoW.

Les situations de combat, quant à elles, utilisent pleinement les voies de communication pour établir une lecture qui se veut au minimum exploratoire, voire configurative. Chaque situation propose un objectif à atteindre, un monde souhaité par les personnages, au joueur, qui doit, en respectant les règles du jeu, établir un plan d'action pour l'atteindre, allant du déploiement des troupes jusqu'à la gestion de ressources. Autrement dit, le joueur n'a pas la possibilité de projeter son propre monde souhaité, et ne peut actualiser que celui proposé. Il peut cependant le faire en employant les moyens qu'il préfère (selon son évaluation), mais il ne peut complètement fixer l'objectif. L'intelligence artificielle de Valkyria Chronicles se donne elle-même un monde souhaité, qui peut être compris aisément à l'aide de la situation narrative (les troupes ennemies attaquent le 
campement du joueur, par exemple). Elle agit à ce titre en tant qu'agent extérieur, nécessairement dans une relation de compétition. Certes, l'intelligence artificielle du jeu n'évalue pas chaque action de façon aussi complexe que Ryan le propose (attribution de valeurs sur trois axes, puis priorisation des mondes accessibles), mais elle demeure un agent soucieux de la rentabilité de ses actions: chaque action inflige un maximum de dommages, ou retarde au maximum le joueur. À noter que la construction d'un monde anticipé n'est pas ici pertinente, puisque le résultat de ce type de construction est fourni d'emblée lors de l'expérience de jeu.

Également, se déroulant non pas en temps réel, mais à tour de rôle, les situations de combat se plient aisément au modèle de l'usage du cybertexte d'Aarseth. Le joueur pose d'abord une série actions, qui sont analysées; de là, l'intelligence artificielle développe un monde anticipé selon ce qu'elle interprète des actions du joueur, puis réoriente ses actions en conséquence. Retour au joueur, qui doit maintenant interpréter les actions du jeu (en faire la synthèse) pour modifier (au besoin) son plan, et le cycle recommence. Il ne s'agit . pas d'un phénomène différent de celui des jeux en temps réel : la seule différence, c'est que chaque étape est délibérée, et permet une plus grande réflexion - et surtout, que le processus est pour nos besoins bien plus transparent.

Les phénomènes de lecture (autres que la lecture interprétative) présents dans Valkyria Chronicles se situent donc, comme nous venons de le voir, essentiellement sur le plan ludique. Nous allons maintenant nous pencher sur les différents mécanismes employés 
par le jeu pour rendre plus rentables certains enchaînements $d^{\prime}$ actions, encadrant de façon douce les actions posées par le joueur.

On trouve dans le jeu de nombreux artifices pour rendre plus rentables certaines séquences d'actions ludiques. L'instrument principal utilisé est la note attribuée à la fin de chaque mission aux efforts du joueur : selon son efficience, la note va de D (la plus basse) à A (la meilleure). Plus la note est bonne, plus le joueur est récompensé en points d'expérience (on peut remarquer ici un élément récurrent dans les jeux à l'étude). Le seul critère observé pour attribuer la note est le nombre de tours employé par le joueur : autrement dit, le jeu n'évalue les méthodes utilisées que par leur pure efficience (pas de moralité imposée, par exemple). Le joueur reste libre alors de configurer son action selon ses préférences, et le jeu les prend en considération pour se réajuster, ne proposant comme notion de rentabilité que la rapidité d'exécution. Il existe d'autres incitatifs (un allié tombé au combat peut mourir s'il n'est pas secouru rapidement) mais celui de l'efficience demeure le plus présent.

Chaque mission offre toutefois des obstacles différents, qui doivent être interprétés judicieusement (dans son monde perçu) par le joueur s'il veut exécuter l'action la plus efficiente : peut-être n'y a-t-il pas de règles morales, mais il existe des impératifs ponctuels rendant plus rentables que d'autres certaines actions, qui demandent donc une souplesse du joueur. Ce dernier demeure tout de même foncièrement libre d'atteindre les objectifs du jeu comme il lui plaît, et peut même ignorer l'efficience s'il ne la juge pas nécessaire, 
disposant ainsi d'une grande latitude sur le plan des actions souhaitées, en autant qu'elles mènent, évidemment, au monde souhaité final.

Les mécanismes de lecture, appliqués à la dimension ludique de Valkyria Chronicles, révèlent une richesse interactive qui fait défaut à sa dimension fictionnelle. Les deux aspects sont ainsi vécus séparément, et utilisent de façon complètement différente le travail de lecture. Le programme est au cœur de la fiction, ne laissant au joueur qu'un rôle interprétatif, tandis que l'expérience de jeu, elle, mise plutôt sur l'échange continu entre jeu et joueur, qui force le jeu à reconfigurer ses actions. En bref, les particularités vidéoludiques de la lecture ne sont utilisées par Valkyria Chronicles que pour bonifier son expérience de jeu. Il ne faut pas croire cependant que la fiction en souffre pour autant: n'allouant que peu d'interaction, elle est conçue de façon traditionnelle, comme un film animé, ce qui lui permet d'utiliser tous les leviers éprouvés de la lecture interprétative rebondissements, tromperies, développement de personnages, etc. - de façon très efficace.

\section{Conclusion}

Lorsque nous avons établi notre cadre d'analyse, nous nous sommes attaqués à deux éléments que nous considérions essentiels à la lecture vidéoludique. Le premier était la notion de lecture ergodique, avancée par Aarseth pour illustrer le travail requis pour approcher hypertextes et cybertextes (catégories au sein desquels nous classons les jeux électroniques) en dehors de l'interprétation. Le second était la notion de rentabilité de la lecture, que nous avons choisi d'exprimer en nous référant au modèle proposé par Ryan 
de l'approche par mondes possibles à la lecture, se déclinant en trois axes, se basant sur une perception incomplète.

À la lumière de notre analyse, nous avons pu voir que les jeux choisis exemplifient différentes approches de la lecture vidéoludique, et de l'encadrement des actions à travers des mécanismes de rentabilité. Fallout 3 nous a proposé un monde fictionnel qui se présente intentionnellement de façon incomplète, obligeant le joueur-agent à redéfinir, à travers sa lecture, sa perception du monde et de ses codes moraux. World of Warcraft : Wrath of the Lich King présentait un monde fictionnel peu interactif, à peine explicité, et misait énormément sur l'interaction entre les joueurs plutôt qu'entre le joueur et le programme afin de générer une expérience complexe de lecture et de jeu. Valkyria Chronicles, finalement, se présente à prime abord comme une fiction traditionnelle, mais sur le plan du jeu permet une interaction assez large (quoique fortement encadrée) entre l'agent-joueur et les agents commandés par le programme.

Dans tous les cas, les mécanismes de rentabilité se ressemblaient: octroi de points d'expérience ou de ressources, qui peuvent être utilisés pour la progression du personnage. Les trois jeux appartenant à la même grande famille des jeux de rôle, il était naturel qu'il y ait un certain recoupement dans les phénomènes de lecture employés. Toutefois, il est étonnant de constater que deux jeux célébrés pour leur scénario (Fallout 3 et Valkyria Chronicles) puissent offrir un rapport à la fiction et à la lecture si différent preuve, s'il en fallait, des vastes possibilités offertes par le jeu électronique. Certaines sont 
peu exploitées (l'intelligence artificielle des jeux étudiés était un peu simpliste, loin de la complexité proposée par Ryan) mais d'autres montrent déjà leur grand potentiel. 


\section{CONCLUSION}

Nous terminions notre introduction en évoquant l'idée que nous allions tenter, au cours de ce mémoire de cerner les qualités propres au jeu électronique, notamment à travers l'analyse de cas de figures, pour dresser un portrait des nouvelles possibilités qu'il peut offrir, et établir un vocabulaire pour parler de la fiction, du récit et de la lecture vidéoludiques. À partir de ce que nous avons vu dans les précédents chapitres, il est maintenant le temps de constater ce que nous en avons dit, et de faire le bilan desdites possibilités du médium.

Notre premier chapitre, consacré à la fiction, nous a permis de réconcilier les notions de jeu et de fiction. Nous avons alors établi que ces deux réalités coexistent à l'intérieur de jeux électroniques à monde fictionnel ${ }^{162}$, et sont constituées par des ensembles structurants de règles, tels que détaillés par la pensée systémique de la fiction ${ }^{163}$, et la littérature concernant le vidéoludique ${ }^{164}$. La cadre d'analyse qui a prévalu pour les études de cas était centré autour de la notion d'intégration des systèmes comme qualité unique au vidéoludique.

L'analyse, donc, cherchait à repérer dans quelle mesure et selon quelles modalités les différents jeux étudiés intégraient fiction et jeu, au sens d'ensembles structurants; afin de

\footnotetext{
162 Notion empruntée à Jesper Juul - Half-Real: Video Games Between Real Rules and Fictional Worlds, dérivée du tableau analytique présenté dans notre premier chapitre.

163 Umberto Eco - Lector in Fabula.

164 Chee Siang Ang - «Rules, gameplay, and narratives in video games ».
} 
créer différents effets d'immersion ${ }^{165}$. Trois approches distinctes sont ressorties de l'analyse: Fallout 3 présentait un fort degré d'intégration, faisant visiblement des efforts pour traduire dans les mécanismes de jeu des éléments ludiques, World of Warcraft : Wrath of the Lich King tendait à délaisser la pure cohérence, délaissant le côté fictionnel afin de raffiner et simplifier les mécanismes de jeu, tandis que Valkyria Chronicles délaissait l'intégration des deux ensembles de règles, laissant le joueur parcourir en parallèle les deux structures.

Notre deuxième chapitre a servi à montrer les possibilités énormes, du point de vue de la structure narrative, offertes par le médium informatique et par extension, le jeu électronique. Empruntant à la pensée littéraire du récitt66, et la contrastant avec les approches du récit vidéoludique ${ }^{167}$, nous en sommes venus à conceptualiser une façon de concevoir le récit (centré autour de l'action) et ses enchaînements possibles qui puisse s'adapter au jeu électronique. De là, nous avons discuté de la place du joueur dans la sélection des actions et des enchaînements, et développé un vocabulaire qui rend compte de l'aspect ludique de l'organisation de l'expérience du récit et du jeu.

L'analyse nous a permis de dégager trois structures bien différentes, cas de figures d'un potentiel bien plus vaste : Fallout 3 illustrant une structure arborescente, World of Warcraft : Wrath of the Lich King présentant une structure modulaire et Valkyria Chronicles offrant une

\footnotetext{
165 Allison McMahan - «Immersion, Engagement and Presence: A Method for Analyzing 3-D Video Games ». 166 Bertrand Gervais - Récits et actions. Pour une théorie de la lecture.

167 Marie-Laure Ryan - «Beyond Myth and Metaphor: The Case of Narrative in Digital Media » et Fotis Jannidis - « Event-Sequences, Plots and Narration in Computer Games».
} 
structure linéaire. Dans tous les cas, la place du joueur s'est montrée variable - il reste néanmoins qu'il se montrait généralement impliqué un minimum dans le processus d'enchaînement de séquences narratives.

Notre dernier chapitre a quant à lui illustré les différences essentielles entre l'acte de lecture textuelle conventionnelle, et le rapport particulier qu'entretient le joueur envers le jeu. En nous basant sur une approche de l'hypertexte et du cybertexte ${ }^{168}$ nous avons pu établir les différents types de « lecture » rendues possibles par le support informatique : interprétative, exploratoire, configurative et textonique, ainsi que leurs modes d'exécution, notamment à l'aide de voies de communication. Afin de mieux cerner de quelle façon s'opère le travail de lecture effectué par le joueur, nous avons également étoffé les notions d'inférences, de rentabilité et de lecture prospective ${ }^{169}$. Aidé du paradigme systémique, nous avons repensé le joueur non seulement en tant que lecteur, mais également en tant qu'agent.

À travers l'examen des jeux choisis, nous avons pu illustrer différents mécanismes de rentabilité encadrant la lecture et l'action des joueurs (le karma de Fallout 3 ou la réputation de World of Warcraft:Wrath of the Lich King, et même l'octroi de gains supplémentaires de ressources de Valkyria Chronicles). Nous nous sommes penchés également sur les voies de communication permettant au joueur de communiquer ses intentions au jeu, et particulièrement sur les dialogues, pour faire ressortir leur importance

168 Espen Aarseth - Cybertext; Perspectives on Ergodic Literature.

169 Marie-Laure Ryan - Possible Worlds, Artificial Intelligence, and Narrative Theory et Umberto Eco - Lector in Fabula. 
dans la définition du joueur en tant que lecteur - plutôt configuratif pour Fallout 3, surtout exploratoire pour World of Warcraft : Wrath of the Lich King et essentiellement interprétatif dans Valkyria Chronicles.

Tout au long de ce mémoire, nous avons développé un vocabulaire propre à la fiction, au récit et à la lecture vidéoludique. La notion d'intégration et de cohérence fictionnelle, d'abord, nous a servi à exprimer le rapport entre jeu et fiction. Nous avons ensuite emprunté les différents procédés d'immersion, qui nous permettaient d'éclaircir $l^{\prime}$ investissement (émotionnel, intellectuel, social) du joueur. Puis nous en sommes venus à associer les termes vidéoludiques de zones, de quêtes et de rencontres aux cadres, situations dramatiques et actions du récit, employant ainsi une terminologie qui rende compte de la spécificité du jeu vidéo. Finalement, nous avons pu étayer les procédés de lecture et de construction de mondes possibles, avec l'inclusion de l'étude des voies de communication, et le détail des éléments régissant la rentabilité de lecture, axes d'évaluation de la possibilité, de la désirabilité et de la moralité. Ce vaste vocabulaire, parfois emprunté, souvent inspiré, nous semble être en mesure de communiquer ce que nous défendions dès le départ : fiction, récit et lecture, s'ils ne sont pas essentiels au jeu électronique, peuvent néanmoins y trouver un mode d'expression unique, avec ses particularités, son style, sa voix - et de là, son paradigme d'analyse.

Si le débat sur la place du narratif dans le jeu électronique est (du moins à notre sens) désormais résolu, le jeu électronique en tant que tel demeure un objet méconnu, malgré sa 
grandissante popularité170. S'il est vrai que «l'apparition d'une nouvelle technologie et d'un nouveau rapport à la composition n'implique pas automatiquement $\mathrm{l}^{\prime}$ apparition d'une nouvelle forme »171, déjà, la possibilité d'en arriver à quelque chose d'innovateur, de créatif et d'artistique est présente dans le jeu électronique. Sa valeur reste encore à prouver, mais ses millions d'adeptes sont là pour le défendre: le critique de cinéma américain Roger Ebert, après avoir réitéré que « les jeux vidéo ne pourraient jamais être un art ${ }^{172}$, a reçu plus de 4500 commentaires et courriels (!) défendant l'opinion contraire. Sa constatation: «Je n'exprimerais jamais une opinion sur un film que je n'ai pas vu. Pourtant j'ai déclaré comme un axiome que les jeux vidéo ne pourraient jamais être un Art. Je le crois toujours, mais je n'aurais jamais du le dire. ${ }^{173}$ La place est maintenant aux chercheurs, aux spécialistes - ce sera à eux de déterminer s'il y a lieu de parler d'un champ artistique vidéoludique, d'une poétique ou d'une stylistique propre au jeu électronique.

\footnotetext{
170 Afin d'illustrer notre propos, le jeu Call of Duty : Modern Warfare 3 (Infinity Ward, Sledgehammer Games et al. - Call of Duty : Modern Warfare 3, Activision, 2011) venait d'établir, au moment d'écrire ces lignes, un record de recettes au lancement, toutes ouvres confondues (cinéma compris) : un milliard de dollars en seize jours. Fabien Déglise - "Le jeu vidéo guerrier désarme le cinéma», Le Devoir.com (15 décembre 2011). <http://www.ledevoir.com/societe/science-et-technologie/338428/le-jeu-video-guerrier-desarme-le-cinema> (21 décembre 2011)

171 Samuel Archibald - Le texte et la technique : la lecture à l'ère des nouveaux médias, p.169.

172 Roger Ebert - «Videogames can never be art», Chicago Sun-Tintes (16 avril 2010). $<$ http://blogs.suntimes.com/ebert/2010/04/video_games_can_never_be_art.html> (21 décembre 2011) (Nous traduisons)

173 Roger Ebert - "Okay, kids, play on my lawn», Chicago Sun-Times (1 juillet 2010). <http://blogs.suntimes.com/ebert/2010/07/okay_kids_play_on_my_lawn.html> (21 décembre 2011) (Nous traduisons)
} 


\section{BIBLIOGRAPHIE}

AARSETH, Espen - Cybertext; Perspectives on Ergodic Literature, John Hopkins University Press, Baltimore, 1997.

ARCHIBALD, Samuel - Le texte et la technique : la lecture à l'heure des nouveaux médias, Thèse de doctorat (sémiologie), Université du Québec à Montréal, 2008. <http://www.archipel.uqam.ca/902/> (14 septembre 2011)

ATARI - Adventure, Atari, 1978.

AUSTEN, Jane - Orgueil et Préjugés, Christian Bourgois Éditeur, Paris, 1979

BETHESDA SOFTWORKS - The Elder Scrolls IV: Oblivion, 2K Games, 2006.

BETHESDA GAME STUDIOS - Fallout 3, Bethesda Softworks, 2008.

BETHESDA GAME STUDIOS - Fallout : Newv Vegas, Obsidian Entertainment, 2010.

BIOWARE - Baldur's Gate, Interplay, 1998.

BLACK ISLE STUDIOS - Fallout, Interplay, 1997.

BLACK ISLE STUDIOS - Fallout 2, Interplay, 1998.

BLIZZARD ENTERTAINMENT - World of Warcraft, Blizzard Entertainment, 2004.

BLIZZARD ENTERTAINMENT - World of Warcraft: The Burning Crusade, Blizzard Entertainment, 2007.

BLIZZARD ENTERTAINMENT - World of Warcraft: Wrath of the Lich King, Blizzard Entertainment, 2008.

BLIZZARD ENTERTAINMENT - World of Warcraft: Cataclysm, Blizzard Entertainment, 2010.

BLIZZARD ENTARTAINMENT - «Classes », World of Warcraft, Cataclysm, (2011). <http://us.battle.net/wow/en/game/class/> (15 septembre 2011)

CAILLOIS, Roger - Les Jeux et les hommes : le masque et le vertige, Éditions Gallimard (Folio Essais), Paris, 1992. (Édition originale 1958) 
DEGLISE, Fabien - "Le jeu vidéo guerrier désarme le cinéma », Le Devoir.com (15 décembre 2011). <http://www.ledevoir.com/societe/science-et-technologie/338428/le-jeuvideo-guerrier-desarme-le-cinema> (21 décembre 2011)

DOUGLAS, Jane Yellowlees - The End of Books - Or Books without End? Reading Interactive Narratives, The University of Michigan Press, Ann Arbor, 2001.

EA CANADA - NHL 10, EA Sports, 2009.

EA TIBURON - NFL Head Coach 09, EA Sports, 2008.

EBERT, Roger - «Videogames can never be art», Chicago Sun-Times (16 avril 2010). <http://blogs.suntimes.com/ebert/2010/04/video_games_can_never_be_art.html>

EBERT, Roger - «Okay, kids, play on my lawn», Chicago Sun-Times (1 juillet 2010). <http://blogs.suntimes.com/ebert/2010/07/okay_kids_play_on_my_lawn.html> (21 décembre 2011)

ECO, Umberto - Lector in fabula; Le rôle du lecteur, Éditons Grasset, Paris, 1985.

«E3 2010: Guiness World Records », SEGA Blog (17 juin 2010). <http:/ / blogs.sega.com/2010/06/17/e3-2010-guinness-world-records/> septembre 2011).

FAHS, Travis - «The Lives and Deaths of the Interactive Movie », IGN (3 mars 2008). <http:/ / retro.ign.com/articles/856/856379p1.html> (11 septembre 2011)

«Fallout 3 Wins The Golden Joysticks», Kotaku (30 octobre 2009). <http://kotaku.com/5393592/fallout-3-wins-the-golden-joysticks> (14 septembre 2011).

FRASCA, Gonzalo - «Ludology meets Narratology: Similitude and differences between (video)games and narrative", Parnasso, No. 3 (1999). <http://www.ludology.org/articles/ludology.htm> (11 septembre 2011)

FRASCA, Gonzalo - Videogames of the Oppressed - Videogames as a Means for Critical Thinking and Debate, Thèse de maîtrise (Information, Design and Technology), $\begin{array}{llll}\text { Georgia Institute of Technology, } 2001 . & \end{array}$ <http://www.ludology.org/articles/thesis/FrascaThesisVideogames.pdf> septembre 2011)

FRASCA, Gonzalo - «Simulation versus Narrative: Introduction to Ludology » in The Video Game Theory Reader, Routledge, New York, 2003, p.221-235. 
FRASCA, Gonzalo - Ludologists love stories too: notes from a debate that never took place, présenté à la conference DIGRA (2003). <http://www.ludology.org/articles/Frasca_LevelUp2003.pdf> (11 septembre 2011)

GERVAIS, Bertrand - Récits et actions. Pour une théorie de la lecture, Les Éditions du Préambule, Longueuil, 1990.

HERMAN, David, JAHN, Manfred et RYAN, Marie-Laure - The Routledge Encyclopedia of Narrative Theory, Routledge, New York, 2005.

HUIZINGA; Johan - Homo Ludens: Essai sur la fonction sociale du jeu, Éditions Gallimard, Paris, 1951.

IGDA Online Games SIG - 2004 Persistent Worlds Whitepaper, 2004. $<$ http://www.igda.org/online/IGDA_PSW_Whitepaper_2004.pdf> (11 septembre 2011)

«IGN 2008 Game of the Year», IGN (16 janvier 2009). $<$ http://video.ign.com/dor/articles/944244/ign-game-of-the-year-2008revealed/videos/igngoty2008_winner_011609.html> (14 septembre 2011)

INFINITY WARD, SLEDGEHAMMER GAMES, et al. - Call of Duty : Modern Warfare 3, Activision, 2011.

JANNIDIS, Fotis - «Event-Sequences, Plots and Narration in Computer Games", Dichtung Digital (2006). <http://dichtungdigital.mewi.unibas.ch/2006/01/Jannidis/index.htm> (14 septembre 2011)

JENKINS, Henry - «Game Design as a Narrative Architecture », Henry Jenkins blog. $<$ http://web.mit.edu/cms/People/henry3/games\&narrative.html $>$ (14 septembre 2011)

JENKINS, Henry-' "The future of Sandbox Games", Confessions of an Aca-Fan (10 décembre 2007). <http://henryjenkins.org/2007/12/gambit.html> (11 septembre 2011)

JUUL, Jesper - A Clash between Game and Narrative, University of Copenhagen Press, Copenhagen, 2001.

JUUL, Jesper - Half-Real: Video Games Between Real Rules and Fictional Worlds, MIT Press, Cambridge, 2005.

JUUL, Jesper - A Casual Revolution: Reinventing Video Games and Their Players, MIT Press, Cambridge, 2009. 
KENT, Steve L. - The Ultimate History of Video Games: From Pong to Pokemon - The Story Behind the Craze That Touched Our Lives and Changed the World, Three Rivers Press, New York, 2001.

MCMAHAN, Allison - «Immersion, Engagement and Presence: A Method for Analyzing 3-D Video Games » in The Video Game Theory Reader, Routledge, New York, 2003, p.67-86.

Metacritic. <http://www.metacritic.com/> (14 septembre 2011)

MICRO FORTE - Fallout Tactics: Brotherhood of Steel, 14 Degrees East, 2001.

MURRAY, Janet - Hamlet on the Holodeck: The Future of Narrative in Cyberspace, MIT Press, Cambridge, 1998.

PENNAC, Daniel - Comme un roman, Gallimard, 1992.

QUEST - Tactics Ogre; Let Us Cling Together, Atlus, 1995.

ROWLING, Joanne K. - Harry Potter and the Philosopher's Stone, Bloomsbury, Londres, 1997.

RYAN, Marie-Laure - «Beyond Myth and Metaphor: The Case of Narrative in Digital Media", Game Studies, Vol. 1, No. 1 (Juillet 2001). <http://www.gamestudies.org/0101/ryan/> (11 septembre 2011)

RYAN, Marie-Laure - Possible Worlds, Artificial Intelligence, and Narrative Theory, Indiana University Press, Bloomington \& Indianapolis, 1991.

SAWYER, Josh - "I thought I could organize freedom. How Scandinavian of me », RPG Codex, (16 décembre 2002). <http://www.rpgcodex.net/content.php?id=24> (14 septembre 2011)

SEGA - Valkyria Chronicles, Sega, 2008.

SEGA - Valkyria Chronicles II, Sega, 2010.

SEGA - Valkyria Chronicles III, Sega, 2011.

SPIESS, Kevin - «Swedish reviewer: Fallout 3 "Oblivion with guns", gives game a $81 \%$ », Neoseeker (8 octobre 2008). <http://www.neoseeker.com/news/8987-swedishreviewer-fallout-3-oblivion-with-guns-gives-game-a-81/> (15 septembre 2011)

SQUARESOFT - Final Fantasy III, SquareSoft, 1994. 
SQUARESOFT - Final Fantasy Tactics, SCEA, 1998.

SUITS, Bernard, The Grasshopper: Games, Life and Utopia, University of Toronto Press (1978).

TOTILO, Stephen - «Sink or Swim? The Game Designer's Conundrum », Kotaku (2 juillet 2009). $\quad<$ http:// kotaku.com/5306343/sink-or-swim-the-game-designersconundrum $>$ (15 septembre 2011)

WOODCOCK, Bruce - «MMOG Active Suscriptions 200,000+ », MMOGCHART.COM (2008). <http://www.mmogchart.com/Chart1.html> (15 septembre 2011)

YEE, Nick - PARC PlayOn Group. <http://www.nickyee.com/index-playon.html> (14 septembre 2011)

«2009 Interactive Achievement Awards», Academy of Interactive Arts and Sciences (2009). $<$ http://www.interactive.org/awards/2009_12th_awards.asp> (14 septembre 2011).

«9th Annual Game Developers Choice Awards», Game Developers Choice Awvards (2009). $<$ http://www.gamechoiceawards.com/archive/gdca_9th.html $\quad$ (14 septembre 2011) 